\title{
Geographic distribution of phlebotomine sandfly species (Diptera: Psychodidae) in Central-West Brazil
}

\author{
Paulo Silva de Almeida ${ }^{1,2}$, Andrey José de Andrade ${ }^{3}$, Alan Sciamarelli', Josué Raizer', \\ Jaqueline Aparecida Menegatti ${ }^{4}$, Sandra Cristina Negreli Moreira Hermes ${ }^{4}$, \\ Maria do Socorro Laurentino de Carvalho ${ }^{5}$, Rodrigo Gurgel-Gonçalves ${ }^{3 /+}$
}

\author{
${ }^{1}$ Faculdade de Ciências Biológicas e Ambientais, Universidade Federal da Grande Dourados, Dourados, MS, Brasil \\ ${ }^{2}$ Laboratório Regional de Entomologia, Núcleo Regional de Saúde, Secretaria de Estado de Saúde, Dourados, MS, Brasil \\ ${ }^{3}$ Laboratório de Parasitologia Médica e Biologia de Vetores, Área de Patologia, Faculdade de Medicina, Universidade de Brasília, Brasília, \\ DF, Brasil ${ }^{4}$ Laboratório de Entomologia, Secretaria de Saúde do Estado do Mato Grosso, Cuiabá, MT, Brasil \\ ${ }^{5}$ Diretoria de Vigilância Ambiental em Saúde, Secretaria de Estado da Saúde do Distrito Federal, Brasília, DF, Brasil
}

This study updates the geographic distributions of phlebotomine species in Central-West Brazil and analyses the climatic factors associated with their occurrence. The data were obtained from the entomology services of the state departments of health in Central-West Brazil, scientific collections and a literature review of articles from $1962-$ 2014. Ecological niche models were produced for sandfly species with more than 20 occurrences using the Maxent algorithm and eight climate variables. In all, 2,803 phlebotomine records for 127 species were analysed. Nyssomyia whitmani, Evandromyia lenti and Lutzomyia longipalpis were the species with the greatest number of records and were present in all the biomes in Central-West Brazil. The models, which were produced for 34 species, indicated that the Cerrado areas in the central and western regions of Central-West Brazil were climatically more suitable to sandflies. The variables with the greatest influence on the models were the temperature in the coldest months and the temperature seasonality. The results show that phlebotomine species in Central-West Brazil have different geographical distribution patterns and that climate conditions in essentially the entire region favour the occurrence of at least one Leishmania vector species, highlighting the need to maintain or intensify vector control and surveillance strategies.

Key words: Phlebotominae - ecological niche modelling - Maxent - Cerrado

Leishmaniases primarily affect people living in poverty and they are neglected tropical diseases worldwide. According to the World Health Organization (2010), 350 million people are exposed to leishmaniases and two million cases occur every year. In the Americas, most of the cases occur in Brazil (Alvar et al. 2012). Between 20012012, 3,321 confirmed cases of visceral leishmaniasis (VL) were reported in Central-West Brazil. The majority (78\%) were reported in Mato Grosso do Sul (MS), followed by Mato Grosso (MT), Goiás (GO) and the Federal District (DF). In the same period, there were 49,932 confirmed cases of American tegumentary leishmaniasis (ATL) in Central-West Brazil, most of which (82\%) occurred in MT, followed by GO, MS and DF [Information System on Notifiable Diseases (saude.gov.br/sinanweb)].

In total, 989 phlebotomine species have been identified globally, of which 531 were recorded in the Americas and 277 in Brazil (Shimabukuro \& Galati 2010, Andrade et al. 2013, Ladeia-Andrade et al. 2014, Oliveira et al. 2015, Vilela et al. 2015). Various studies have reported a great species

doi: 10.1590/0074-02760140462

Financial support: CAPES, CNPq

+Corresponding author: rgurgel@unb.br

Received 5 December 2014

Accepted 27 February 2015 richness of sandflies in Central-West Brazil, particularly in MT (106 spp), followed by MS (57 spp), GO (41 spp) and DF (27 spp) (Young \& Duncan 1994, Martins et al. 2002, de Oliveira et al. 2006, Galati et al. 2006, Andrade Filho et al. 2007, Missawa \& Maciel 2007, de Almeida et al. 2010b, 2013a, de Carvalho et al. 2010, SES/MT 2013). However, only 20 phlebotomine species are associated with the transmission of Leishmania in Brazil, including seven species in Central-West Brazil (MS 2006, 2010, Ready 2013).

Lutzomyia longipalpis (Lutz \& Neiva 1912) is the main vector of Leishmania infantum in Latin America and it has a widespread distribution in Brazil (Deane \& Deane 1962, Lanzaro et al. 1993, Sherlock 1996, Aguiar \& Medeiros 2003, Galati 2003). Lutzomyia cruzi (Mangabeira, 1938 ) has been implicated as the L. infantum vector in some regions of MS (Galati et al. 1997, Santos et al. 1998) and MT (Missawa et al. 2011). Lutzomyia forattini Galati, Rego, Nunes \& Teruya, 1985, Lutzomyia almerioi Galati \& Nunes, 1999, Nyssomyia antunesi (Coutinho, 1939), Migonemyia migonei (França, 1920) and Nyssomyia neivai (Pinto, 1926) might be associated with VL transmission in Brazil (Galati et al. 1997, Lainson \& Rangel 2003, Saraiva et al. 2009, Carvalho et al. 2010, Dias et al. 2013).

Nyssomyia whitmani (Antunes \& Coutinho, 1939) has widespread distribution in Latin America and is the most important ATL vector in Brazil (Young \& Duncan 1994, Galati 2003, Peterson \& Shaw 2003, da Costa et al. 2007). In addition, Bichromomyia flaviscutellata (Mangabeira, 1942), Ny. neivai and Nyssomyia intermedia (Lutz \& Neiva, 1912) have been incriminated as ATL vectors 
in various regions of Brazil (Shaw \& Lainson 1968, Condino et al. 1998, Marcondes et al. 2005, 2009, Massafera et al. 2005, Andrade Filho et al. 2007, Silva et al. 2008, Dorval et al. 2010, de Almeida et al. 2013a).

Various studies have analysed the epidemiological importance and ecological aspects of sandflies in Central-West Brazil. However, the geographic distribution and dispersal potential of phlebotomine species must be described and analysed to understand the geographic dimensions of the risk of leishmaniases transmission. Ecological niche modelling (ENM) could be used in this context to estimate the potential geographic distribution of species, based on known occurrence records (Peterson 2006, Peterson et al. 2011). ENM has been used to estimate sandfly distribution and factors related to leishmaniases transmission (Peterson \& Shaw 2003, Gebre-Michael et al. 2004, Peterson et al. 2004, Nieto et al. 2006, Zeilhofer et al. 2008, Colacicco-Mayhugh et al. 2010, de Oliveira et al. 2012, de Almeida et al. 2013b, Moo-Llanes et al. 2013, Quintana et al. 2013, Samy et al. 2014). The aim of this study was to analyse the geographic distribution of phlebotomine species in Central-West Brazil as well as the climatic factors associated with their occurrence.

\section{MATERIALS AND METHODS}

Study area - Central-West Brazil covers an area of $1,606,371 \mathrm{~km}^{2}$ and consists of the following four federal units: MT, MS, GO and DF. The region is divided into 467 municipalities, most of which are in GO [Brazilian Institute of Geography and Statistics (ibge.gov.br/ cidades)], which has a population of $14,993,194$, found predominantly in urban areas $(89 \%)$. Geographically, Central-West Brazil is formed by a central and a southern plateau and the Pantanal plain. The Cerrado, the predominant biome, covers the greatest area; however, the other biomes include the Pantanal, Atlantic Forest and Amazon Forest (Fig. 1). The climate is tropical with the following two well-defined seasons: a rainy summer between October-March and a dry winter between April-September.
Distribution data - The phlebotomine occurrence data were obtained from the entomology services in the state departments of health (MT, MS, GO and DF) and correspond to captures conducted between 1996-2014. In addition, the literature data between 1962-2014 were reviewed (Martins et al. 1962, 1978, 2002, Galati et al. 1985, 1989, 1997, 2001, 2006, Carvalho et al. 1989, Azevedo et al. 2002, de Oliveira et al. 2003, 2006, Braga-Miranda et al. 2006, Dorval et al. 2006, 2009, 2010, Andrade Filho et al. 2007, Missawa \& Dias 2007, Missawa \& Maciel 2007, Silva et al. 2007, 2008, Missawa et al. 2008, Andrade et al. 2009, de Almeida et al. 2010a, b, 2013a, b, Paiva et al. 2010, Amaral et al. 2011, Mestre et al. 2011, Alves et al. 2012, Queiroz et al. 2012, Santos et al. 2013, Thies et al. 2013). The records from scientific collections in speciesLink (splink.cria.org. $\mathrm{br} /$ ), a distributed information system that combines primary data from scientific collections in real time and in the Museum of Zoology of the University of São Paulo were analysed as well. The taxonomic classification used follows Galati (2003) and the abbreviations of the sandfly genera are those proposed by Marcondes (2007).

Species occurrence in the biomes - To analyse the phlebotomine occurrence in the biomes in CentralWest Brazil, the species distribution data and the limits of the biomes in the region were superimposed using QGIS 2.6. The relative occurrence (the number of records in the biome/total number of records for the species in Central-West Brazil) was then calculated and represented graphically using Excel.

$E N M$ - The phlebotomine species records were georeferenced with a confidence level of $<5 \mathrm{~km}$ and an approximate accuracy of $0.01^{\circ}$. The geographic coordinates of named places were obtained from an online gazetteer (fallingrain.com/world) and the data were organised in spreadsheets. The species occurrence database was reviewed in ArcGIS to avoid duplicate records at the spatial resolution used and obvious errors of georeferencing (e.g., points in the ocean) or identification (out dated taxonomic arrangements).

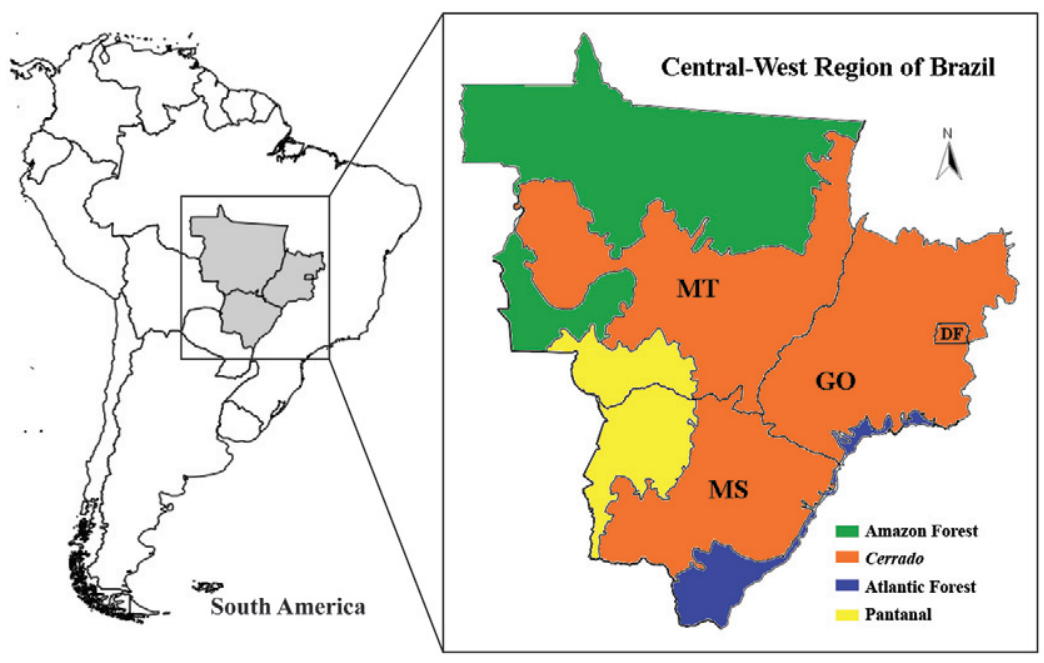

Fig. 1: study area showing the states in Central-West Brazil and the limits of the biomes in this region. DF: Federal District; GO: state of Goiás; MS: state of Mato Grosso do Sul; MT: state of Mato Grosso. 
Potential distribution models were produced for the phlebotomine species with more than 20 records to allow for more accurate modelling (Stockwell \& Peterson 2002). The models were based on the following eight climatic variables: the mean annual temperature, mean diurnal temperature range, temperature seasonality, maximum temperature in the warmest months, minimum temperature in the coldest months, annual precipitation, precipitation in the wettest months and precipitation in the driest months. These data were obtained from the WorldClim project (worldclim.org) and are the result of interpolation of the mean monthly climatic data from meteorological stations over 30-50 years (1950-2000), depending on the data availability at the stations (Hijmans et al. 2005). The eight variables were selected to avoid confounding effects by producing models in an environmental space with an excess of dimensions (Peterson \& Nakazawa 2008). The environmental data used in the analyses had a spatial resolution of $5 \times 5 \mathrm{~km}$ per pixel. The potential distribution models were produced by the maximum entropy method using Maxent, v.3.2.1. (Phillips et al. 2006). The basic parameters proposed by the program were used with 10 replications by bootstrap sub sampling. The occurrence data for the species were separated into two sets, as follows: one set for the model calibration ( $75 \%$ of the points) and the other set for the model evaluation $(25 \%$ of the points). The potential geographic distribution models (the median output grids from Maxent) were imported and edited using the ArcGis 9 program (ESRI).

The model accuracy was assessed by analysing the omission rates associated with the test points (Anderson et al. 2002). The jackknife test implemented in Maxent was used to identify which variables had the greatest influence on the distribution of the recorded phlebotomine species (Phillips et al. 2006). This test measures the predictive effect of each variable in the model by determining the quality of the models produced with only the variable being tested and the quality of those produced with this variable being omitted.

\section{RESULTS}

In all, 2,803 phlebotomine records for 17 genera and 127 species were analysed in Central-West Brazil, as follows: Bichromomyia (2 spp), Brumptomyia (7 spp), Evandromyia (21 spp), Expapillata (1 sp.), Lutzomyia (12 spp), Martinsmyia (2 spp), Micropygomyia (11 spp), Migonemyia (2 spp), Nyssomyia (8 spp), Pintomyia (11 spp), Pressatia (3 spp), Psathyromyia (19 spp), Psychodopygus (13 spp), Trychopygomyia (3 spp), Trichophoromyia (6 spp), Sciopemyia (3 spp) and Viannamyia (3 spp). These fauna correspond to approximately $50 \%$ of all the known phlebotomine species in Brazil. MT was the state with the greatest species richness $(n=108 ; 85 \%)$, followed by MS $(\mathrm{n}=61 ; 48 \%), \mathrm{GO}(\mathrm{n}=39 ; 31 \%)$ and DF $(\mathrm{n}=29$; 23\%). The Evandromyia, Lutzomyia, Psathyromyia and Psychodopygus genera had the greatest species richness.

The Brumptomyia, Evandromyia, Lutzomyia, Nyssomyia, Pintomyia, Psathyromyia, Micropygomyia and Sciopemyia genera were distributed widely throughout Central-West Brazil. The geographical distributions of most of the phlebotomine species analysed in this study are shown in Supplementary Figure.

All 34 species with more than 20 points have at least one record in the Cerrado and 24 (70\%) presented a relative occurrence $>50 \%$ in this biome (Fig. 2). Ten species were more common in the Amazon, particularly Evandromyia bacula, Psychodopygus complexus and Trychopygomyia dasypodogeton. $N y$. neivai and Lu. cruzi were the species with the highest number of records in the Atlantic Forest and the Pantanal, respectively (Fig. 2).

To study the potential geographic distribution of the phlebotomine species in Central-West Brazil, 2,216 records were analysed, as follows: Bi. flaviscutellata $(\mathrm{n}=$ 62), Brumptomyia brumpti $(\mathrm{n}=55)$, Brumptomyia avellari $(\mathrm{n}=33)$, Ev. bacula $(\mathrm{n}=21)$, Evandromyia carmelinoi $(\mathrm{n}=76)$, Evandromyia evandroi $(\mathrm{n}=98)$, Evandromyia lenti $(\mathrm{n}=143)$, Evandromyia saulensis $(\mathrm{n}=62)$, Evandromyia termitophila $(\mathrm{n}=116)$, Evandromyia teratodes

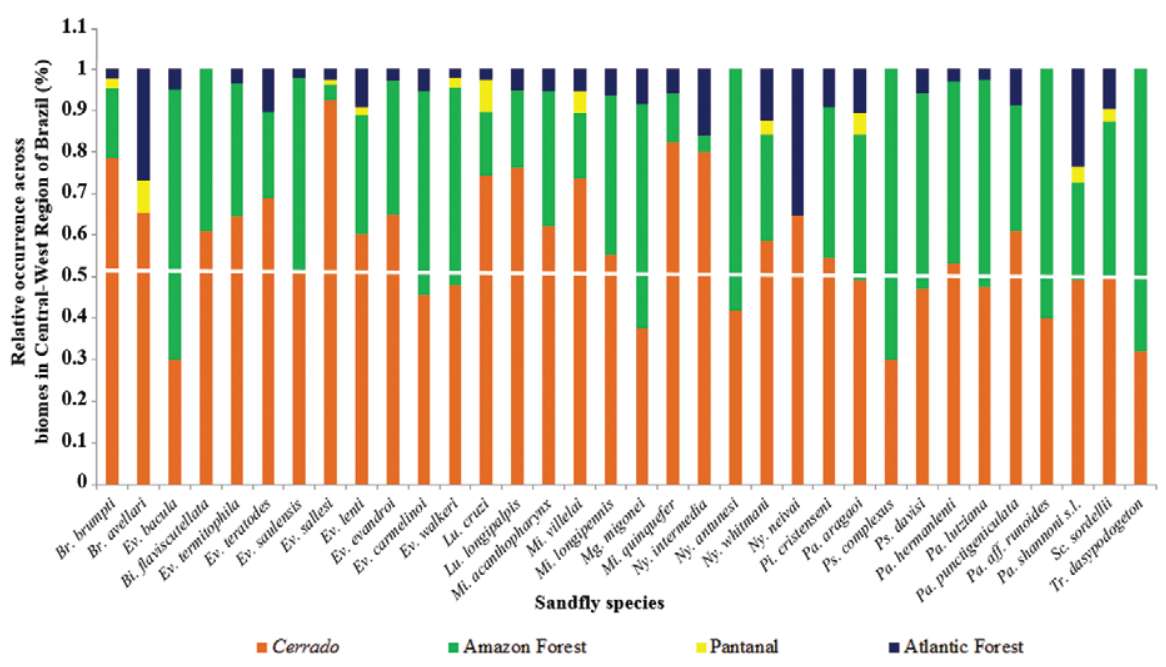

Fig. 2: relative occurrence of 34 phlebotomine species in biomes in Central-West Brazil based on the percentage of known occurrences in each area. The white line indicates a relative frequency of $50 \%$. 

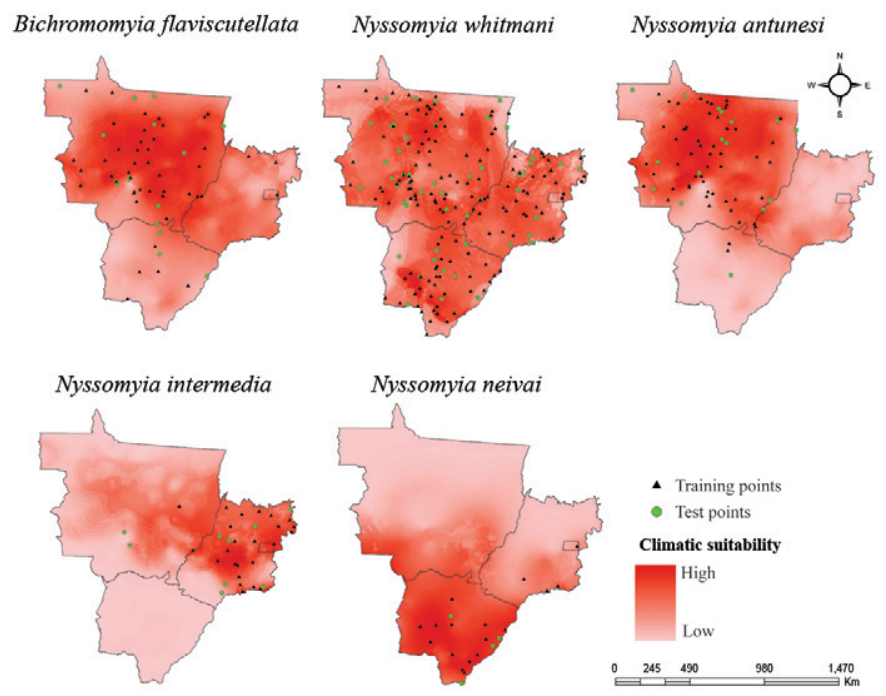

Fig. 3: potential geographic distribution of American tegumentary leishmaniases vectors in Central-West Brazil. The triangles represent the points used to produce the ecological niche model based on eight climate variables. The circles show the points used to evaluate the models. The red scale shows climate suitability for the different species (dark: high; light: low).
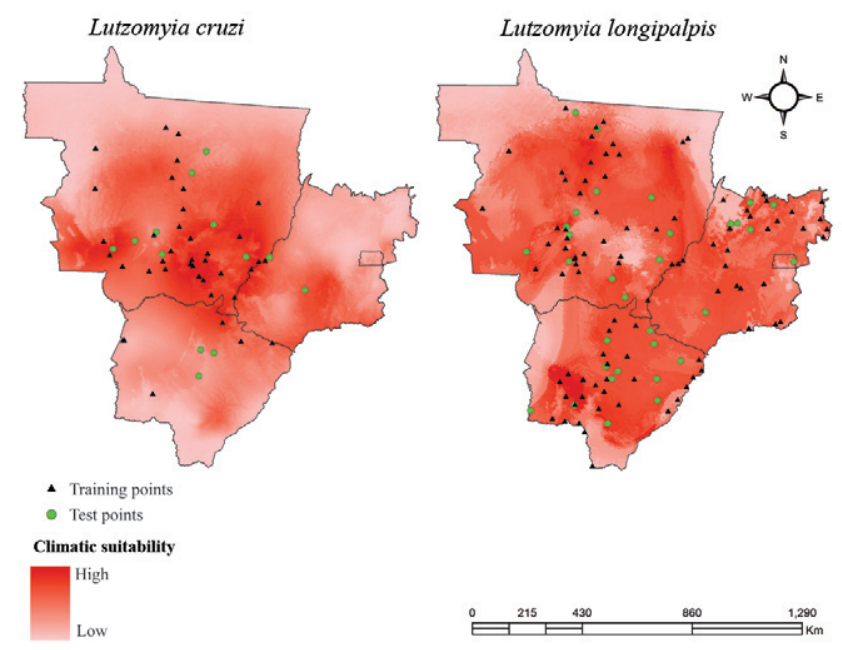

Fig. 4: potential geographic distribution of visceral leishmaniases vectors in Central-West Brazil. The triangles represent the points used to produce the ecological niche model based on eight climate variables. The circles show the points used to evaluate the models. The red scale shows climate suitability for the different species (dark: high; light: low).

$(\mathrm{n}=38)$, Evandromyia walkeri $(\mathrm{n}=61)$, Evandromyia sallesi $(\mathrm{n}=105)$, Lu. cruzi $(\mathrm{n}=52)$, Lu. longipalpis $(\mathrm{n}=129)$, Micropygomyia acanthopharynx $(\mathrm{n}=49)$, Micropygomyia villelai $(=$ Micropygomyia goiana $)(\mathrm{n}=25)$, Micropygomyia longipennis $(\mathrm{n}=62)$, Micropygomyia quinquefer $(\mathrm{n}=22), M g$. migonei $(\mathrm{n}=33), N y$. antunesi $(\mathrm{n}=71), N y$. intermedia $(\mathrm{n}=34), N y$. neivai $(\mathrm{n}=22), N y$. whitmani $(\mathrm{n}=$ 178), Psathyromyia aragaoi $(\mathrm{n}=76)$, Psathyromyia hermanlenti $(\mathrm{n}=85)$, Psathyromyia lutziana $(\mathrm{n}=53)$, Psathyromyia aff. runoides $(\mathrm{n}=46)$, Psathyromyia shannoni "sensu lato" ( $\mathrm{n}=73)$, Psathyromyia punctigeniculata $(\mathrm{n}=$ 61), Pintomyia christenseni $(\mathrm{n}=29)$, Psychodopygus complexus $(\mathrm{n}=27)$, Psychodopygus davisi $(\mathrm{n}=68)$, Sciopemyia sordellii $(\mathrm{n}=123)$ and Ty. dasypodogeton $(\mathrm{n}=28)$. The ecological niche models of most of these species and their geographic coordinates are shown in the Supplementary Figure and Table, respectively.

Ny. whitmani was distributed widely in all the states and had the potential to occur throughout the entire territory of Central-West Brazil (Fig. 3). Bi. flaviscutellata and $N y$. antunesi were common in MT, where the climate was more suitable to these species. However, Bi. flaviscutellata had high potential to occur in southern GO as well. The distributions of $N y$. neivai and $N y$. intermedia were more restricted to MS and GO, respectively (Fig. 3). Lu. longipalpis had a broader geographic distribution than $\mathrm{Lu}$. cruzi. Areas in northern GO, north-western MT and southern MS were not highly suitable for $\mathrm{Lu}$. cruzi (Fig. 4). 
The temperatures in the coldest months and temperature seasonality were the variables with the greatest influence on the models, according to the jackknife tests. Most of the test points for the species were included in the areas predicted by the ENMs. All the models had omission errors $<1 \%$ and could therefore be validated.

\section{DISCUSSION}

This study updated the geographic distributions of phlebotomine species in Central-West Brazil, which account for approximately one-half of all the known species recorded in Brazil. The results show that phlebotomine species have different geographic distribution patterns in this region and that nearly all the areas of Central-West Brazil have climate conditions that favour the occurrence of at least one of these species. The distribution patterns show that sandflies are found more frequently in the Cerrado areas and that temperature seasonality and temperature in the coldest months are the climate variables that have the greatest influence on the species distribution.

The phlebotomine species list of Central-West Brazil was updated. Missawa \& Maciel (2007) and SES/ MT (2013) recorded 106 species in MT, which is fewer than the 108 species registered in this study. In MS, de Oliveira et al. (2006), Galati et al. (2006) and de Almeida et al. (2010b) reported 57 species, which is fewer than the 61 species registered here. Martins et al. (2002) detected 41 species in GO, whereas 47 were found in this study. This study did not find new records of sandfly species in Central-West Brazil. The differences between the numbers of species in the previous lists and in this study are in part because the records of the health departments of municipalities are generally unpublished. In addition, we included the lists of Martins et al. (1978), which is sometimes overlooked in the literature and even the latest revision of Galati (2014), which updates the species records in the Brazilian states. The higher species richness in MT is probably related to the size of this state and the variety of the biomes (the Cerrado, Amazon Forest and Pantanal) and transition zones, which could favour diversification of phlebotomine fauna, as observed for triatomine species (Pereira et al. 2013). According to Sábio et al. (2014), $P a$. shannoni do not occur in Brazil and, based on this taxonomic review of the shannoni series, the specimens recorded in Central-West Brazil is Psathyromyia bigeniculata (Floch \& Abonnenc, 1941). The records of Lutzomyia cruciata, Pintomyia andina, Psathyromyia lanei, Psathyromyia ruparupa, Psychodopygus nigaraguensis and Viannamyia caprina require confirmation. Moreover, Lutzomyia gomezi, Micropygomyia vonatzingeni, Evandromyia cortelezzii and Pintomyia kuscheli could be incorrectly registered in the CWB because they are morphologically similar to Lutzomyia sherlocki, Micropygomyia oswaldoi, Evandromyia corumbaensis and Pintomyia fischeri or Pintomyia pessoai, respectively (EAB Galati, unpublished observations). Specimens identified as Sciopemyia microps and Pa. runoides can be new species; in the present study they are considered as Sciopemyia aff. microps and Pa. aff. runoides (AJ Andrade, unpublished observations). The occurrence records in the present study are according to identified species by health services and formally published. The revision of specimens deposited in scientific collections should elucidate these possible taxonomic problems.

$N y$. whitmani showed a widespread geographic distribution in Central-West Brazil, corroborating the findings of other studies (Young \& Duncan 1994, Galati 2003, Peterson \& Shaw 2003). This species is one of the commonest in artificial environments in Brazil. Its presence in an area is positively correlated with deforestation and it occurs primarily in municipalities with lower economic development indexes (Galati et al. 2006, da Costa et al. 2007, Missawa et al. 2008, Zeilhofer et al. 2008, de Almeida et al. 2013b). Ny. intermedia occurs predominantly in GO and DF; however, this study indicated that areas in western MT are climatically suitable and could therefore favour dispersal of this species within this state. This species has a widespread distribution in the Southeast Region of Brazil (Peterson \& Shaw 2003). The climatic conditions in southern Central-West Brazil (particularly in MS) were suitable for $N y$. neivai and not for $N y$. intermedia. The distribution of $N y$. intermedia and $N y$. neivai should be revised in future studies because they are similar species and identification errors could cause it to be difficult to map their geographic distributions correctly (Marcondes 1996, Andrade Filho et al. 2003, 2007). Bi. flaviscutellata had great potential to occur in southern GO within the Cerrado biome, for which there are few records of this species. According to Young and Duncan (1994), Bi. flaviscutellata is found predominantly in the Amazon Region. Our results show that it is frequently found in the Cerrado as well and that it probably disperses along the gallery forests in this biome. $N y$. antunesi, Ty. dasypodogeton and Ps. complexus were found predominantly in northern MT and had a significant relative occurrence in the Amazon biome, which is in agreement with Young and Duncan (1994).

Lu. longipalpis occurred in all the biomes in CentralWest Brazil, confirming its great adaptability to different environments (Deane \& Deane 1962, Lanzaro et al. 1993, Sherlock 1996, Aguiar \& Medeiros 2003, Galati 2003, de Almeida et al. 2013b). Lu. cruzi occurred predominantly in MT and MS, confirming the findings of Missawa and Lima (2006). However, the niche model revealed high climate suitability for $\mathrm{Lu}$. cruzi in southern GO. Further studies in these areas are required to confirm the presence of $L u$. cruzi. Although not involved in the transmission of leishmaniases, Ev. lenti was widespread in Central-West Brazil, as previously reported by Young and Duncan (1994) and Galati (2003).

The variables with the greatest influence on the models were the temperatures in the coldest months and temperature seasonality, as in the study of Lu. longipalpis in MS by de Almeida et al. (2013b). These results are in agreement with those of Guzmán and Tesh (2000) and highlight the fundamental importance of temperature for the development and occurrence of phlebotomines. However, biotic and socioeconomic variables influence the occurrence of these insects as well (Zeilhofer et al. 2008). Therefore, further studies analysing the geographic distribution of phlebotomines under environmental and climate-change scenarios are required (Moo-Llanes et al. 2013). 
Considerable efforts were made in this study to establish a comprehensive, representative, up-to-date database of phlebotomine species in Central-West Brazil. However, although the data were gathered from a variety of sources (e.g., scientific articles, museums and books), some records will inevitably have been overlooked, as an inherent limitation of geographic distribution studies. Furthermore, taxonomic issues might have had an effect on the occurrence data, maps and models described here. The database spatial resolution in this study could limit the accuracy of the ENMs. Models based on low-resolution data tend to overestimate the species distribution limits (Seo et al. 2009). The effects of these uncertainties related to low resolution tend to be smaller in studies on a continental or regional scale (Wiens et al. 2009).

Finally, this study could facilitate the development of surveillance and control strategies for leishmaniases in Central-West Brazil. Areas that are climatically suitable for the species discussed in this study and are without any confirmed occurrence should be investigated in future phlebotomine surveys. The maps produced here could serve as reference to future studies on sandflies and are the first step to developing an Atlas of Phlebotominae in Brazil, with the geographical distribution of all the species, which could be useful in academic studies and health services.

\section{ACKNOWLEDGEMENTS}

To Dr Marcos Takashi Obara (University of Brasilia), for reviewing the paper.

\section{REFERENCES}

Aguiar GM, Medeiros WM 2003. Distribuição e habitats. In EF Rangel, R Lainson (orgs.), Flebotomíneos do Brasil, Fiocruz, Rio de Janeiro, p. 207-255.

Alvar J, Vélez ID, Bern C, Herrero M, Desjeux P, Cano J, Jannin J, den Boer M, WHO Leishmaniasis Control Team 2012. Leishmaniasis worldwide and global estimates of its incidence. PLoS ONE 7: e35671.

Alves GB, Oshiro ET, Leite MC, Melão AV, Ribeiro LM, Mateus NLF, Brazil RP, Andrade Filho JD, de Oliveira AG 2012. Phlebotomine sand flies fauna (Diptera: Psychodidae) at rural settlements in the municipality of Cáceres, state of Mato Grosso, Brazil. Rev Soc Bras Med Trop 45: 437-443.

Amaral AFS, Varjão JR, Silva GB, Arrais-Silva WW 2011. Phlebotomine fauna (Diptera: Psychodidae: Phlebotominae) in a residential area and in a fragment of savanna vegetation in the municipality of Pontal do Araguaia, Mato Grosso, Brazil. Rev Bras Parasitol Vet 20: 165-167.

Anderson RP, Gómez-Laverde M, Peterson AT 2002. Geographical distributions of spiny pocket mice in South America: insights from predictive models. Global Ecol Biogeogr 11: 131-141.

Andrade AJ, Shimabukuro PHF, Galati EAB 2013. On the taxonomic status of Phlebotomus breviductus Barretto, 1950 (Diptera: Psychodidae: Phlebotominae). Zootaxa 3734: 477-484.

Andrade Filho JD, Galati EAB, Falcão AL 2003. Redescription of Nyssomyia intermedia (Lutz \& Neiva, 1912) and Nyssomyia neivai (Pinto, 1926) (Diptera: Psychodidae). Mem Inst Oswaldo Cruz 98: 1059-1065.

Andrade Filho JD, Galati EAB, Falcão AL 2007. Nyssomyia intermedia (Lutz \& Neiva, 1912) and Nyssomyia neivai (Pinto, 1926) (Diptera: Psychodidae: Phlebotominae) geographical distribution and epidemiological importance. Mem Inst Oswaldo Cruz 102: 481-487.
Andrade ROA, Nunes VLB, Galati EAB, Arruda CCP, Santos MFC, Rocca MLGE, Aquino RB 2009. Estudo epidemiológico das leishmanioses em área de turismo ambiental e ecoturismo, estado de Mato Grosso do Sul, 2006-2007. Rev Soc Bras Med Trop 42: 488-493.

Azevedo ACR, Souza NA, Meneses CRV, Costa WA, Costa SM, Lima JB, Rangel EF 2002. Ecology of sand flies (Diptera: Psychodidae: Phlebotominae) in the north of the state of Mato Grosso, Brazil. Mem Inst Oswaldo Cruz 97: 459-464.

Braga-Miranda LC, Miranda M, Galati EAB 2006. Phlebotomine fauna in a rural area of the Brazilian Pantanal. Rev Saude Publica 40: 324-326.

Carvalho MESD, Heloísa ES, Naves HAM 1989. Contribuição ao conhecimento da fauna flebotomínica do estado de Goiás e Distrito Federal. II - 1986-1987. Rev Patol Trop 18: 7-14.

Carvalho MR, Valença HF, Silva FJ, Pita-Pereira D, Pereira TA, Britto C, Brazil RP, Brandão-Filho S 2010. Natural Leishmania infantum infection in Migonemyia migonei (França, 1920) (Diptera: Psychodidae: Phlebotominae) the putative vector of visceral leishmaniasis in Pernambuco state, Brazil. Acta Trop 116: 108-110.

Colacicco-Mayhugh M, Masuoka P, Grieco J 2010. Ecological niche model of Phlebotomus alexandri and P. papatasi (Diptera: Psychodidae) in the Middle East. Int J Health Geogr 9: 1-9.

Condino ML, Sampaio SM, Henriques LF, Galati EAB, Wanderley DMV, Corrêa FMA 1998. Leishmaniose tegumentar americana: flebotomíneos de área de transmissão no município de Teodoro Sampaio, região sudoeste do estado de São Paulo, Brasil. Rev Soc Bras Med Trop 31: 355-360.

da Costa SM, Cechinel M, Bandeira V, Zannuncio JC, Lainson R, Rangel EF 2007. Lutzomyia (Nyssomyia) whitmani s.l. (Antunes \& Coutinho, 1939) (Diptera: Psychodidae: Phlebotominae): geographical distribution and the epidemiology of American cutaneous leishmaniasis in Brazil - Mini-review. Mem Inst Oswaldo Cruz 102: 149-153.

de Almeida PS, Leite JA, Araújo AD, Batista MP, Touro RBS, Araújo VS, Souza EJ, Rodrigues JB, Oliveira GA, Santos JV, Faccenda O, Andrade Filho JD 2013a. Fauna of phlebotomine sand flies (Diptera, Psychodidae) in areas with endemic American cutaneous leishmaniasis in the state of Mato Grosso do Sul, Brazil. Rev Bras Entomol 57: 105-112.

de Almeida PS, Minzão ER, Minzão L, Silva SR, Ferreira AD, Faccenda O, Andrade Filho JD 2010a. Aspectos ecológicos de flebotomíneos (Diptera: Psychodidae) em área urbana do município de Ponta Porã, estado de Mato Grosso do Sul. Rev Soc Bras Med Trop 43: 723-727.

de Almeida PS, Nascimento JC, Ferreira AD, Minzão LD, Portes F, Miranda AM, Faccenda O, Andrade Filho JD 2010b. Espécies de flebotomíneos (Diptera: Psychodidae) coletadas em ambiente urbano em municípios com transmissão de leishmaniose visceral do estado de Mato Grosso do Sul, Brasil. Rev Bras Entomol 54: 304-310.

de Almeida PS, Sciamarelli A, Batista PM, Ferreira AD, Nascimento J, Raizer J, Andrade Filho JD, Gurgel-Gonçalves R 2013b. Predicting the geographic distribution of Lutzomyia longipalpis (Diptera: Psychodidae) and visceral leishmaniasis in the state of Mato Grosso do Sul, Brazil. Mem Inst Oswaldo Cruz 108: 992-996.

de Carvalho MSL, Bredt A, Meneghin ERS, de Oliveira C 2010. Flebotomíneos (Diptera: Psychodidae) em áreas de ocorrência de leishmaniose tegumentar americana no Distrito Federal, Brasil, 2006 a 2008. Epidemiol Serv Saúde 19: 227-237.

de Oliveira AG, Andrade Filho JD, Falcão AL, Brazil RP 2003. Estudo de flebotomíneos (Diptera, Psychodidae) na zona urbana da Cidade de Campo Grande, Mato Grosso do Sul, Brasil, 19992000. Cad Saude Publica 19: 933-944. 
de Oliveira AG, Galati EAB, de Oliveira O, de Oliveira GR, Espindola IAC, Dorval MEC, Brazil RP 2006. Abundance of Lutzomyia longipalpis (Diptera: Psychodidae: Phlebotominae) and urban transmission of visceral leishmaniasis in Campo Grande, state of Mato Grosso do Sul, Brazil. Mem Inst Oswaldo Cruz 101: 869-874.

de Oliveira EF, Silva EA, Fernandes CES, Paranhos Filho AC, Gamarra RM, Ribeiro AA, Brazil RP, de Oliveira AG 2012. Biotic factors and occurrence of Lutzomyia longipalpis in endemic area of visceral leishmaniasis, Mato Grosso do Sul, Brazil. Mem Inst Oswaldo Cruz 107: 396-401.

Deane LM, Deane MP 1962. Visceral leishmaniasis in Brazil: geographical distribution and transmission, São Paulo. Rev Inst Med Trop 4: 198-212.

Dias ES, Michalsky EM, Nascimento JC, Ferreira EC, Valadão JL, Fortes-Dias CL 2013. Detection of Leishmania infantum, the etiological agent of visceral leishmaniasis, in Lutzomyia neivai, a putative vector of cutaneous leishmaniasis. J Vector Ecol 38: 193-196.

Dorval MEC, Alves TP, Cristaldo G, Rocha HC, Alves MA, Oshiro ET, Oliveira AG, Brazil RP, Galati EAB, Cunha RV 2010. Sand fly captures with Disney traps in area of occurrence of Leishmania (Leishmania) amazonensis in the state of Mato Grosso do Sul, mid-western Brazil. Rev Soc Bras Med Trop 43: 491-495.

Dorval MEC, Cristaldo G, da Rocha HC, Alves TP, Alves MA, Oshiro ET, de Oliveira AG, Brazil RP, Galati EAB, da Cunha RV 2009. Phlebotomine fauna (Diptera: Psychodidae) of an American cutaneous leishmaniasis endemic area in the state of Mato Grosso do Sul, Brazil. Mem Inst Oswaldo Cruz 104: 695-702.

Dorval MEC, Oshiro ET, Cupollilo E, Castro ACC, Alves TP 2006. Occurrence of American tegumentary leishmaniasis in the Mato Grosso do Sul state associated to the infection for Leishmania (Leishmania) amazonensis. Rev Soc Bras Med Trop 39: 43-46.

Galati EAB 2003. Classificação de Phlebotominae. In EF Rangel, R Lainson (eds.), Flebotomíneos do Brasil, Fiocruz, Rio de Janeiro, p. 23-51.

Galati EAB 2014. Apostila de bioecologia e identificação de Phlebotominae (Diptera, Psychodidae). Available from: fsp.usp.br/egalati/.

Galati EAB, Nunes VLB, Boggiani PC, Dorval MEC, Cristaldo G, Rocha HC, Oshiro ET, Damasceno-Júnior GA 2006. Phlebotomines (Diptera: Psychodidae) in forested areas of the Serra da Bodoquena, state of Mato Grosso do Sul, Brazil. Mem Inst Oswaldo Cruz 101: 175-193.

Galati EAB, Nunes VLB, Dorval MEC, Cristaldo G, Rocha HC, Gonçalves-Andrade RM, Naufel G 2001. Attractiveness of black Shannon trap for phlebotomines. Mem Inst Oswaldo Cruz 96: 641-647.

Galati EAB, Nunes VLB, Oshiro ET, Rego Jr FA 1989. Nova espécie de Phlebotominae, Lutzomyia corumbaensis, sp. n. (Diptera, Psychodidae) do complexo Lutzomyia cortelezzii. Rev Bras Entomol 33: 465-475.

Galati EAB, Nunes VLB, Rêgo-Júnior FA, Oshiro ET, Chang MR 1997. Estudo de flebotomíneos (Diptera: Psychodidae) em foco de leishmaniose visceral no estado de Mato Grosso do Sul, Brasil. Rev Saude Publica 31: 378-390.

Galati EAB, Rego Jr FA, Nunes VL, Teruya E 1985. Fauna flebotomínica do município de Corumbá, Mato Grosso do Sul, Brasil, e descrição de Lutzomyia forattinii, sp. n. (Diptera, Psychodidae, Phlebotominae). Rev Bras Entomol 29: 261-266.

Gebre-Michael T, Malone J, Balkew M, Ali A, Berhe N, Hailu A, Herzi AA 2004. Mapping the potential distribution of Phlebotomus martini and P. orientalis (Diptera: Psychodidae), vectors of kala-azar in East Africa by use of geographic information systems. Acta Trop 90: 73-86.
Guzmán H, Tesh RB 2000. Effects of temperature and diet on the growth and longevity of phlebotomine sand flies (Diptera: Psychodidae). Biomedica 20: 190-199.

Hijmans J, Cameron SE, Parra JL, Jones PG, Jarvis A 2005. Very high resolution interpolated climate surfaces for global land areas. Int J Climatol 25: 1965-1978.

Ladeia-Andrade S, Fe NF, Sanguinette CC, Andrade Filho JD 2014. Description of Trichophoromyia uniniensis, a new phlebotomine species (Diptera: Psychodidae: Phlebotominae) of Amazonas state, Brazil. Parasit Vectors 7: 400.

Lainson R, Rangel EF 2003. Ecologia das leishmanioses. In EF Rangel, R Lainson (orgs.), Flebotomíneos do Brasil, Fiocruz, Rio de Janeiro, p. 291-309.

Lanzaro GC, Ostrovska K, Herrero MV, Lawyer PG, Warburg A 1993. Lutzomyia longipalpis is a species complex: genetic divergence and interspecific hybrid sterility among three populations. Am J Trop Med Hyg 48: 839-847.

Marcondes CB 1996. A redescription of Lutzomyia (Nyssomyia) intermedia (Lutz \& Neiva, 1912) and resurrection of L. neivai (Pinto, 1926) (Diptera, Psychodidae, Phlebotominae). Mem Inst Oswaldo Cruz 91: 457-462.

Marcondes CB 2007. A proposal of generic and subgeneric abbreviations for phlebotomine sand flies (Diptera: Psychodidae: Phlebotominae) of the world. Entomol News 118: 351-356.

Marcondes CB, Bittencourt IA, Stoco PH, Eger I, Grisard EC, Steindel M 2009. Natural infection of Nyssomyia neivai (Pinto, 1926) (Diptera: Psychodidae, Phlebotominae) by Leishmania (Viannia) spp in Brazil. Trans R Soc Trop Med Hyg 103: 1093-1097.

Marcondes CB, Conceição MBF, Portes MGT, Simão BP 2005. Phlebotomine sandflies in a focus of dermal leishmaniasis in the eastern region of the Brazilian state of Santa Catarina - preliminary results (Diptera: Psychodidae). Rev Soc Bras Med Trop 38: 353-355.

Martins AV, Falcão AL, Silva JE 1962. Nota sobre os flebotomíneos do estado de Goiás com a descrição de duas espécies novas e da fêmea de Lutzomyia longipennis (Barreto, 1946) e a redescrição do macho da L. evandroi (Costa Lima \& Antunes, 1936) (Diptera: Psychodidae). Rev Bras Malariol Doencas Trop 14: 379-394.

Martins AV, Willians P, Falcão AL 1978. American sandflies (Diptera: Psychodidae, Phlebotominae), Academia Brasileira de Ciências, Rio de Janeiro, 195 pp.

Martins F, Silva LG, Bezera WA, Maciel IJ, Silva HHG, Lima CG, Cantuária PB, Ramos OS, Ribeiro JB, Santos AS 2002. Diversidade e frequência da fauna flebotomínea (Diptera: Psychodidae) em áreas com transmissão de leishmaniose no estado de Goiás. Rev Patol Trop 31: 211-224.

Massafera R, Silva AM, Carvalho AP, Santos DR, Galati EAB, Teodoro U 2005. Fauna de flebotomíneos do município de Bandeirantes, no estado do Paraná. Rev Saude Publica 39: 571-577.

Mestre GLC, Ribeiro ALM, Miyazaki RD, Rodrigues JSV, Almeida ABPF, Sousa VRF, Missawa NA 2011. Phlebotomine sand flies and canine infection in areas of human visceral leishmaniasis, Cuiabá, Mato Grosso. Rev Bras Parasitol Vet 20: 228-234.

Missawa NA, Dias ES 2007. Phlebotomine sand flies (Diptera: Psychodidae) in the municipality of Várzea Grande: an area of transmission of visceral leishmaniasis in the state of Mato Grosso, Brazil. Mem Inst Oswaldo Cruz 102: 913-918.

Missawa NA, Lima GBM 2006. Distribuição espacial de Lutzomyia longipalpis (Lutz \& Neiva, 1912) e Lutzomyia cruzi (Mangabeira, 1938) no estado de Mato Grosso. Rev Soc Bras Med Trop 39: 337-340. 
Missawa NA, Maciel GB 2007. List of species in the genus Lutzomyia, França, 1924 (Psychodidae, Phlebotominae) from the state of Mato Grosso. Rev Soc Bras Med Trop 40: 11-14.

Missawa NA, Maciel GBML, Rodrigues H 2008. Distribuição geográfica de Lutzomyia (Nyssomyia) whitmani (Antunes \& Coutinho, 1939) no estado de Mato Grosso. Rev Soc Bras Med Trop 41: 369-373.

Missawa NA, Veloso MAE, Lima GBM, Michalsky EM, Dias ES 2011. Evidência de transmissão de leishmaniose visceral por Lutzomyia cruzi no município de Jaciara, estado de Mato Grosso, Brasil. Rev Soc Bras Med Trop 44: 76-78.

Moo-Llanes D, Ibarra-Cerdeña CN, Rebollar-Téllez EA, Ibáñez-Bernal S, González C, Ramsey JM 2013. Current and future niche of north and central American sand flies (Diptera: Psychodidae) in climate change scenarios. PLoS Negl Trop Dis 7: e2421.

MS - Ministério da Saúde 2006. Manual de vigilância e controle da leishmaniose visceral, DVE/SVS/MS, Brasília, 122 pp.

MS - Ministério da Saúde 2010. Manual de vigilância da leishmaniose tegumentar americana, SVS/MS, Brasília, $180 \mathrm{pp}$.

Nieto P, Malone JB, Bavia ME 2006. Ecological niche modeling for visceral leishmaniasis in the state of Bahia, Brazil, using genetic algorithm for rule-set prediction and growing degree day-water budget analysis. Geospat Health 1: 115-126.

Oliveira AG, Sanguinette CC, Almeida PS, Andrade Filho JD 2015. Description of Evandromyia (Aldamyia) orcyi, a new phlebotomine species (Diptera: Psychodidae: Phlebotominae) from the state of Mato Grosso do Sul, Brazil. Parasit Vectors 8: 233.

Paiva BR, Oliveira AG, Dorval MEMC, Galati EAB, Malafronte RS 2010. Species-specific identification of Leishmania in naturally infected sand flies captured in Mato Grosso do Sul state, Brazil. Acta Trop 115: 126-130.

Pereira JM, de Almeida PS, de Sousa AV, de Paula AM, Machado RB, Gurgel-Gonçalves R 2013. Climatic factors influencing triatomine occurrence in Central-West Brazil. Mem Inst Oswaldo Cruz 108: 335-341.

Peterson AT 2006. Ecologic niche modeling and spatial patterns of disease transmission. Emerg Infect Dis 12: 1822-1826.

Peterson AT, Nakazawa Y 2008. Environmental data sets matter in ecological niche modelling: an example with Solenopsis invicta and Solenopsis richteri. Glob Ecol Biogeogr 17: 135-144.

Peterson AT, Pereira RS, Neves VFC 2004. Using epidemiological survey data to infer geographic distributions of leishmaniasis vector species. Rev Soc Bras Med Trop 37: 10-14.

Peterson AT, Shaw J 2003. Lutzomyia vectors for cutaneous leishmaniasis in Southern Brazil: ecological niche models, predicted geographic distributions and climate change effects. Int J Parasitol 33: 919-931.

Peterson AT, Soberón J, Pearson RG, Anderson RP, Martínez-Meyer E, Nakamura M, Araújo MB 2011. Ecological niches and geographic distributions, Princeton University Press, Princeton, 314 pp.

Phillips SJ, Anderson RP, Schapire RE 2006. Maximum entropy modeling of species geographic distributions. Ecol Model 190: 231-259.

Queiroz MFM, Varjão JR, Moraes SC, Salcedo GE 2012. Analysis of sandflies (Diptera: Psychodidae) in Barra do Garças, state of Mato Grosso, Brazil, and the influence of environmental variables on the vector density of Lutzomyia longipalpis (Lutz \& Neiva, 1912). Rev Soc Bras Med Trop 45: 313-317.

Quintana M, Salomón O, Guerra R, de Grosso ML, Fuenzalida A 2013. Phlebotominae of epidemiological importance in cutaneous leishmaniasis in northwestern Argentina: risk maps and ecological niche models. Med Vet Entomol 27: 39-48.
Ready PD 2013. Biology of phlebotomine sand flies as vectors of disease agents. Annu Rev Entomol 58: 227-250.

Sábio PB, Andrade AJ, Galati EAB 2014. Assessment of the taxonomic status of some species included in the shannoni complex, with the description of a new species of Psathyromyia (Diptera: Psychodidae: Phlebotominae). J Med Entomol 51: 331-341.

Samy AM, Campbell LP, Peterson AT 2014. Leishmaniasis transmission: distribution and coarse-resolution ecology of two vectors and two parasites in Egypt. Rev Soc Bras Med Trop 47: 57-62.

Santos MFC, Ribolla PEM, Alonso DP, Andrade-Filho JD, Casaril AE, Ferreira AMT, Fernandes CES, Brazil RP, Oliveira AG 2013. Genetic structure of Lutzomyia longipalpis populations in Mato Grosso do Sul, Brazil, based on microsatellite markers. PLoS ONE 8: e74268.

Santos SO, Arias J, Ribeiro AA, Hoffmann MP, Freitas RU, Malacco MAF 1998. Incrimination of Lutzomyia cruzi as a vector of American visceral leishmaniasis. Med Vet Entomol 12: 315-317.

Saraiva L, Carvalho GML, Quaresma PF, Lima ACVMR, Falcão AL, Andrade Filho JD 2009. Natural infection of Nyssomyia neivai (Pinto, 1926) and Evandromyia sallesi (Galvao \& Coutinho, 1939) (Diptera: Psychodidae) by Leishmania infantum chagasi Cunha and Chagas, 1937 in Minas Gerais, Brazil. J Med Entomol 49: 1159-1163.

Seo C, Thorne JH, Hannah L, Thuiller W 2009. Scale effects in species distribution models: implications for conservation planning under climate change. Biol Lett 23: 39-43.

SES/MT - Secretaria de Estado de Saúde de Mato Grosso 2013. Relatório das espécies de flebotomíneos detectados em atividades de vigilância entomológica em Mato Grosso no periodo de 1996 a 2013, Superintendência de Vigilância em Saúde/Coordenadoria de Vigilância em Saúde Ambiental/Gerência de Núcleos de Apoio em Vigilância em Saúde Ambiental, Cuiabá, 51 pp.

Shaw JJ, Lainson R 1968. Leishmaniasis in Brazil: II. Observations on enzootic rodent leishmaniasis in the lower Amazon Region The feeding habitats of the vector, Lutzomyia flaviscutellata, in reference to man, rodents and other animals. Trans $R$ Soc Trop Med Hyg 62: 396-405.

Sherlock IA 1996. Ecological interactions of visceral leishmaniasis in the state of Bahia, Brazil. Mem Inst Oswaldo Cruz 91: 671-683.

Shimabukuro PHF, Galati EAB 2010. Checklist of Phlebotominae (Diptera, Psychodidae) from São Paulo state, Brazil, with notes on their geographical distribution. Biota Neotrop 11: 1-20.

Silva AM, Camargo NJ, Santos DR, Massafera R, Ferreira AC, Postal C, Cristóvão EC, Konolsaisen Jr JJ, Bisetto C, Perinazo R, Teodoro U, Galati EAB 2008. Diversidade, distribuição e abundância de flebotomíneos (Diptera: Psychodidae) no Paraná. Neotrop Entomol 37: 209-225.

Silva EA, Andreotti R, Honer MR 2007. Comportamento de Lutzomyia longipalpis, vetor principal da leishmaniose visceral americana, em Campo Grande, estado de Mato Grosso do Sul. Rev Soc Bras Med Trop 40: 420-425.

Stockwell DRB, Peterson AT 2002. Effects of sample size on accuracy of species distribution models. Ecol Model 148: 1-13.

Thies SF, Ribeiro ALM, Michalsky EM, Miyazaki RD, Fortes-Dias CL, Fontes CJF, Dias ES 2013. Phlebotomine sandfly fauna and natural Leishmania infection rates in a rural area of Cerrado (tropical savannah) in Nova Mutum, state of Mato Grosso in Brazil. Rev Soc Bras Med Trop 46: 293-298.

WHO - World Health Organization 2010. Control of the leishmaniasis. Report of a meeting of the WHO Expert Committee on the control of leishmaniases, WHO, Geneva, 186 pp. 
Vilela ML, Azevedo ACR, Godoy RE 2015. Description of a new phlebotomine species of the Brazilian Cerrado from sandstone caves in Tocantins state, Brazil: Lutzomyia (Lutzomyia) elizabethrangelae sp. n. (Diptera: Psychodidae). J Med Entomol doi: dx.doi.org/10.1093/jme/tjv036.

Wiens JA, Stralberg D, Jongsomjit D, Howell CA, Snyder MA 2009. Niches, models and climate change: assessing the assumptions and uncertainties. Proc Natl Acad Sci USA 106: 19729-19736.
Young DG, Duncan MA 1994. Guide to the identification and geographic distribution of Lutzomyia sand flies in México, the West Indies, Central and South America (Diptera: Psychodidae), American Entomological Institute, Gainesville, 881 pp.

Zeilhofer P, Kummer OP, dos Santos ES, Ribeiro ALM, Missawa NA 2008. Spatial modelling of Lutzomyia (Nyssomyia) whitmani s.l. (Antunes \& Coutinho, 1939) (Diptera: Psychodidae: Phlebotominae) habitat suitability in the state of Mato Grosso, Brazil. Mem Inst Oswaldo Cruz 103: 653-660. 


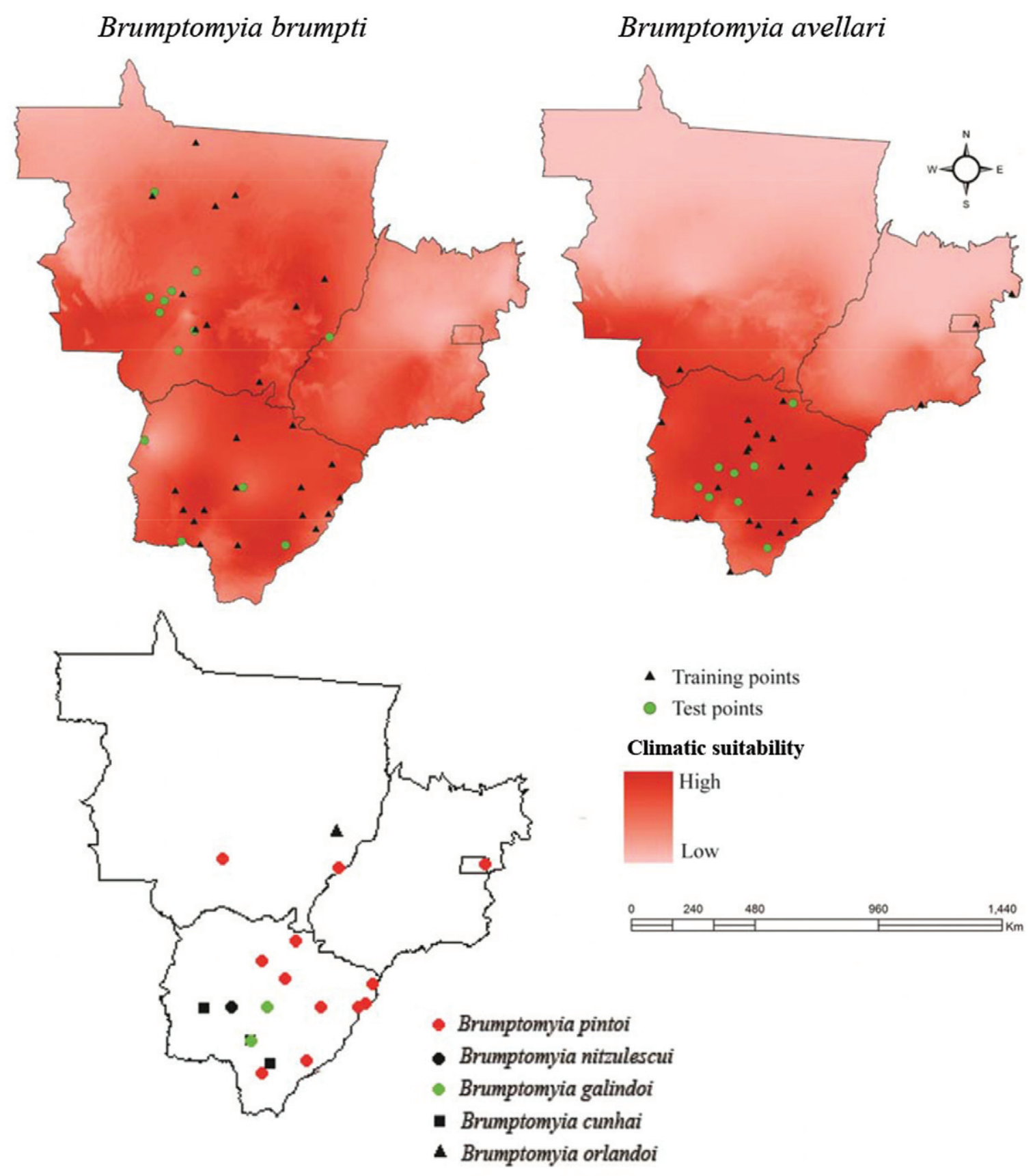



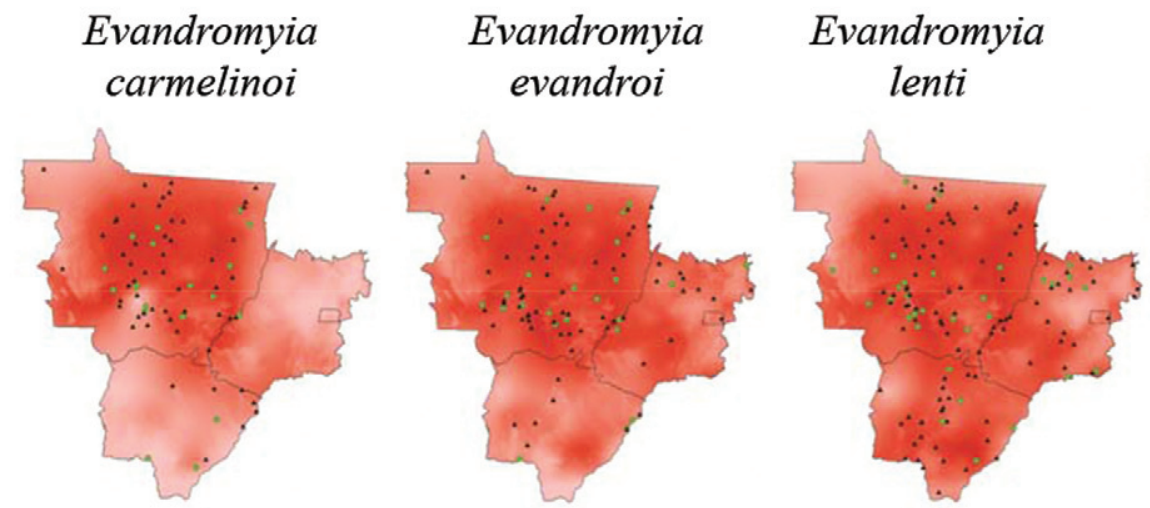

\section{Evandromyia sallesi}

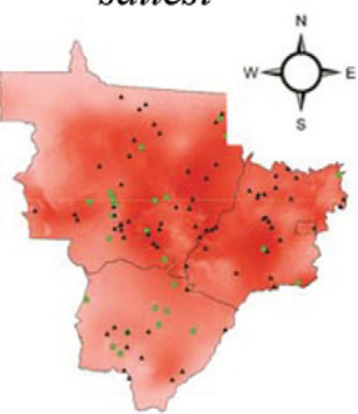

\section{Evandromyia saulensis}

\section{Evandromyia teratodes}

\section{Evandromyia termitophila}

\section{Evandromyia walkeri}
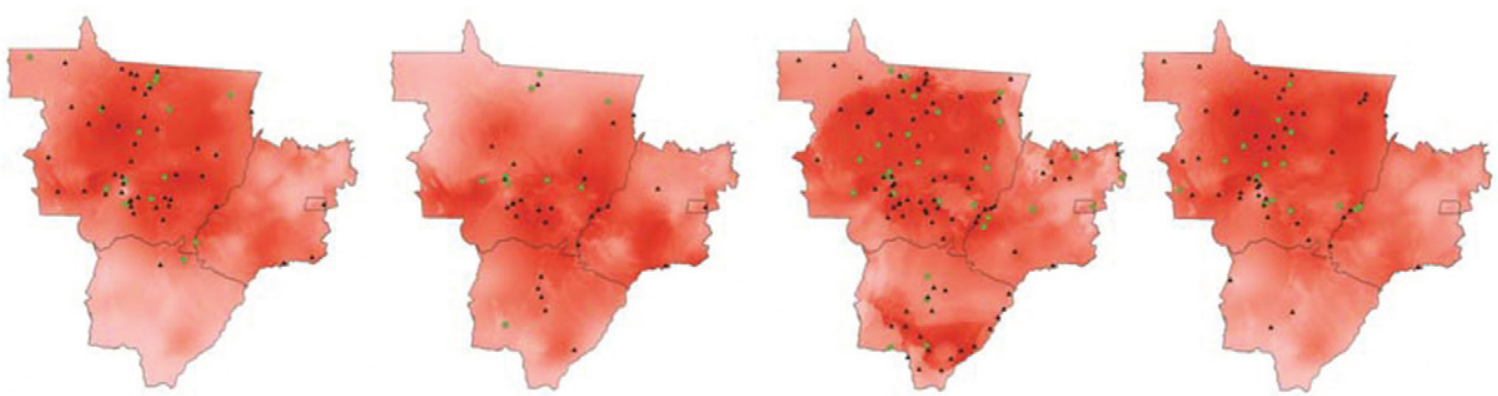

\section{Evandromyia}

bacula
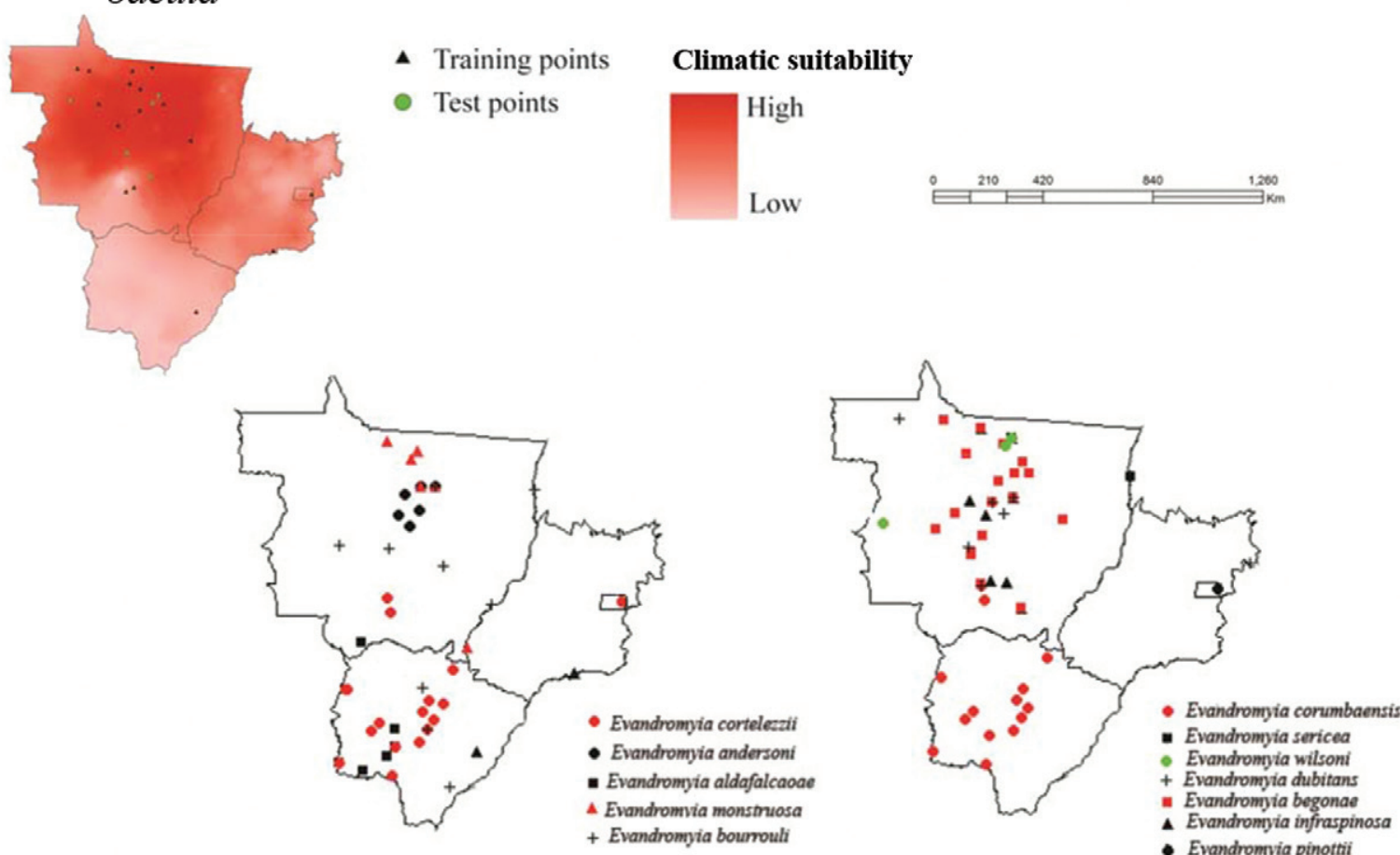

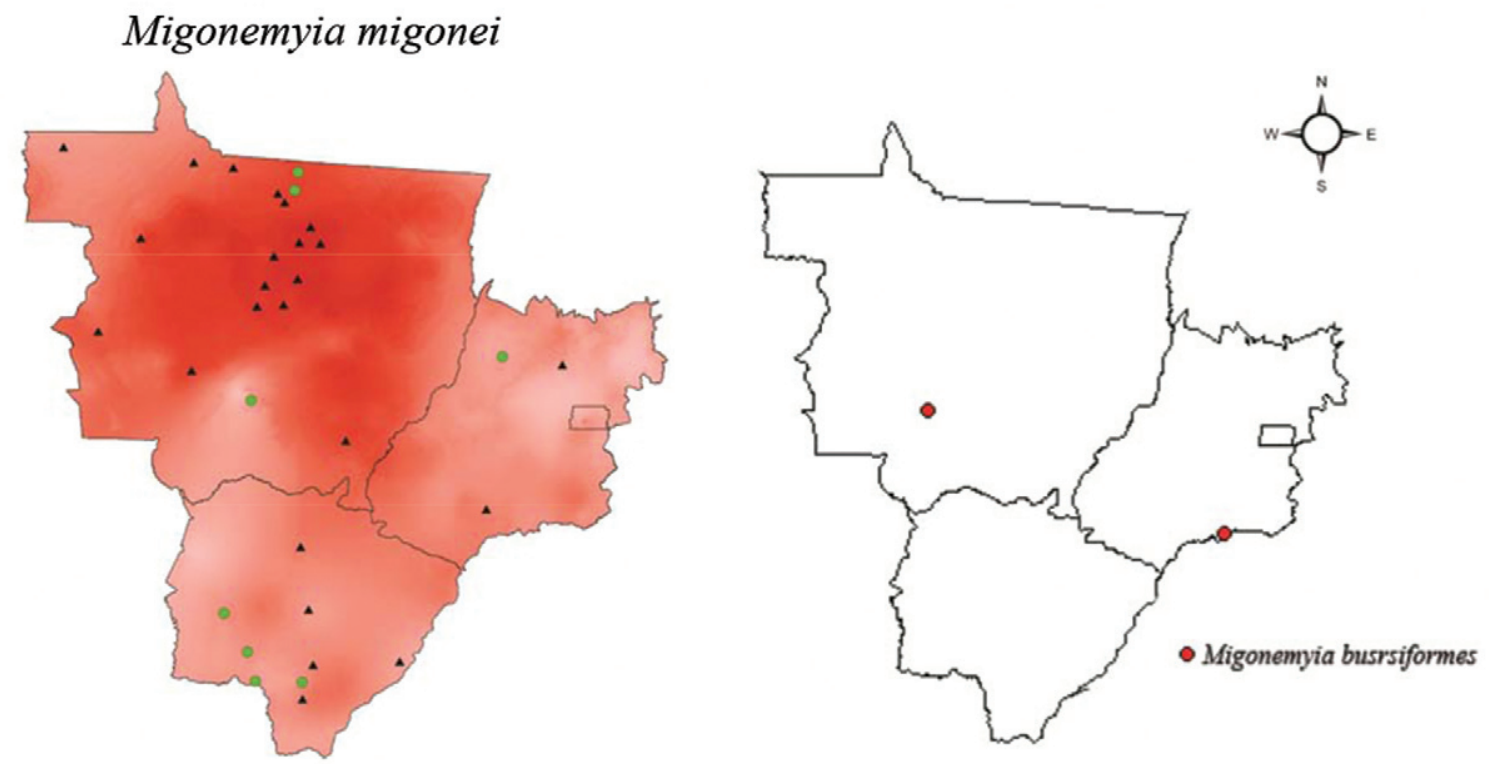

\ Training points

Climatic suitability

- Test points

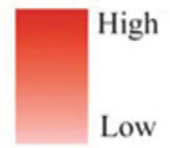

\begin{tabular}{llll}
0 & $210 \quad 420$ & 840 & 1,260 \\
\hline & & 420 & \\
\hline
\end{tabular} 

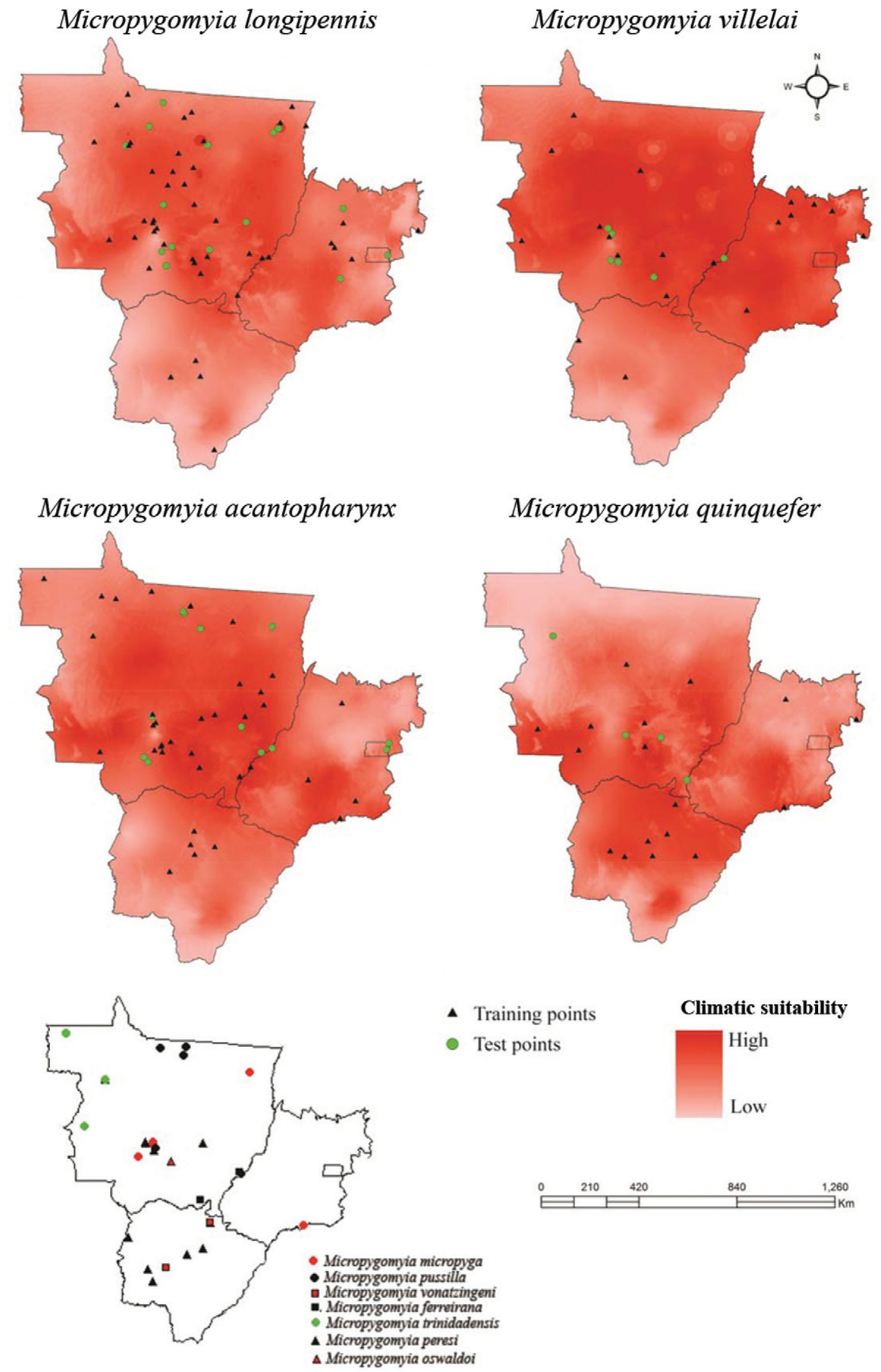

Climatic suitability

- Training points

High

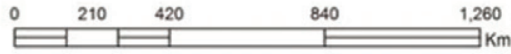



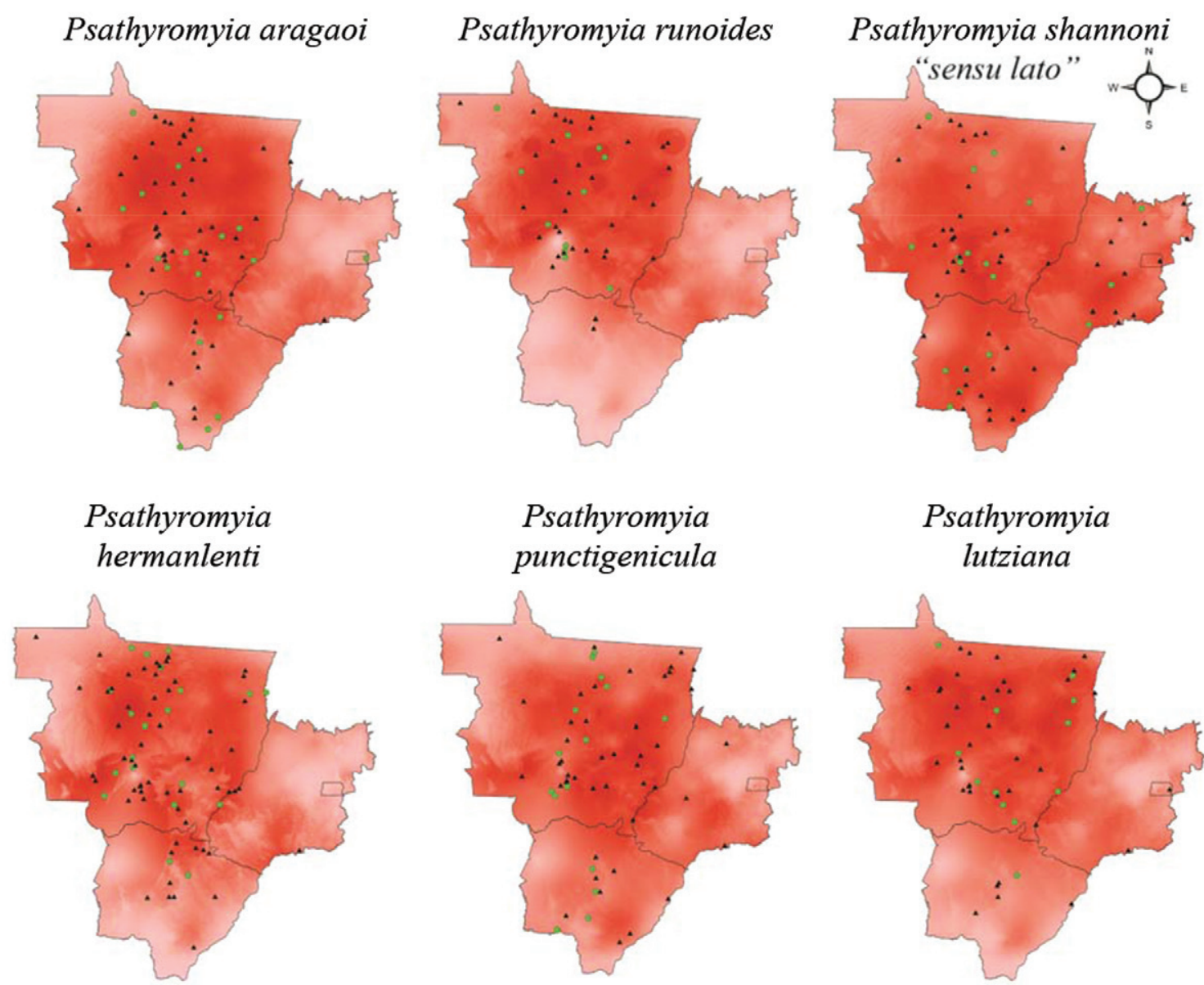

- Training points

Climatic suitability

- Test points
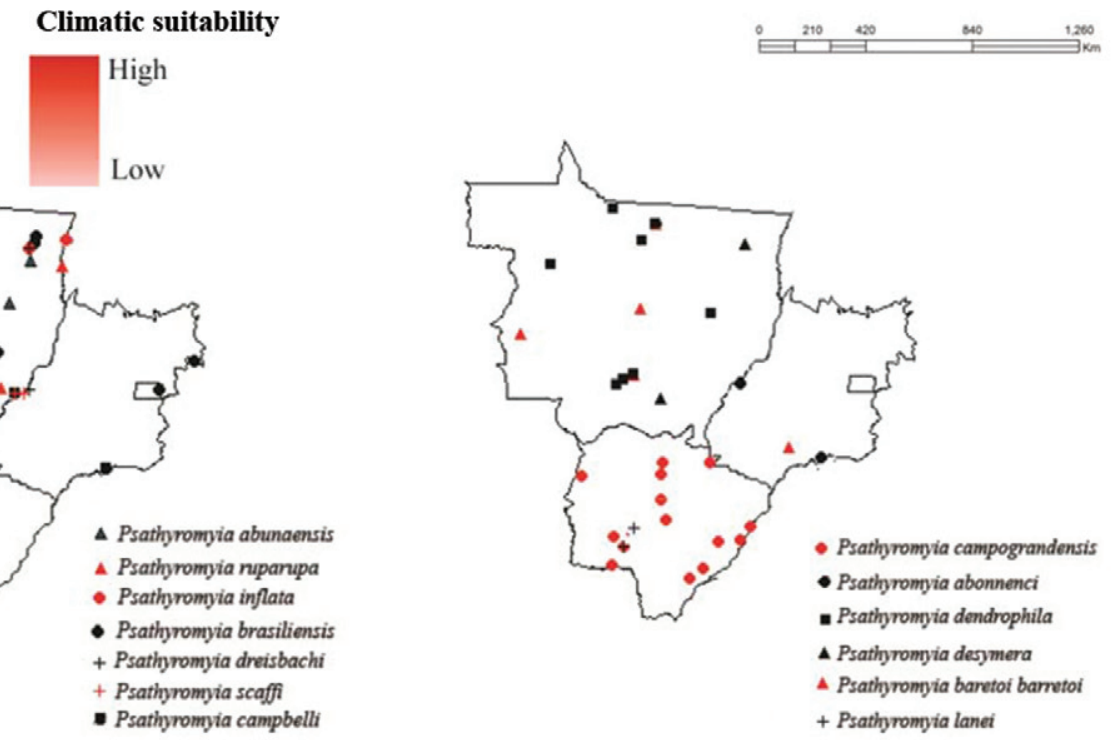

A Psathyromyia abunaensis

A Psathyromyia nupanup

- Psathyromyia inflata

- Psathyromyia brasiliensis

+ Psathyromyia dreisbachi

+ Psathynomyia scaffi

- Psathyromyia campbelli

+ Psathyromyia lanei 


\section{Psychodopygus complexus}

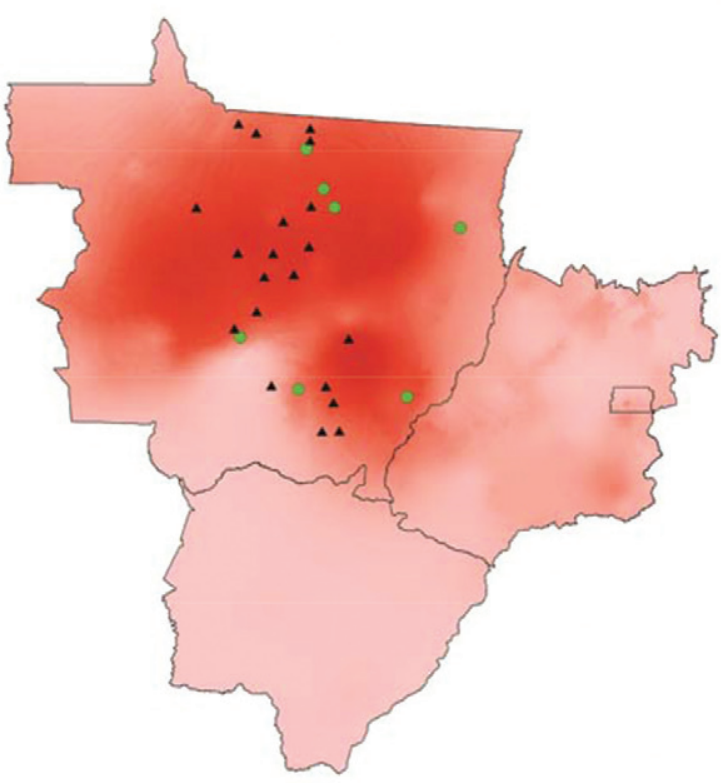

Psychodopygus davisi

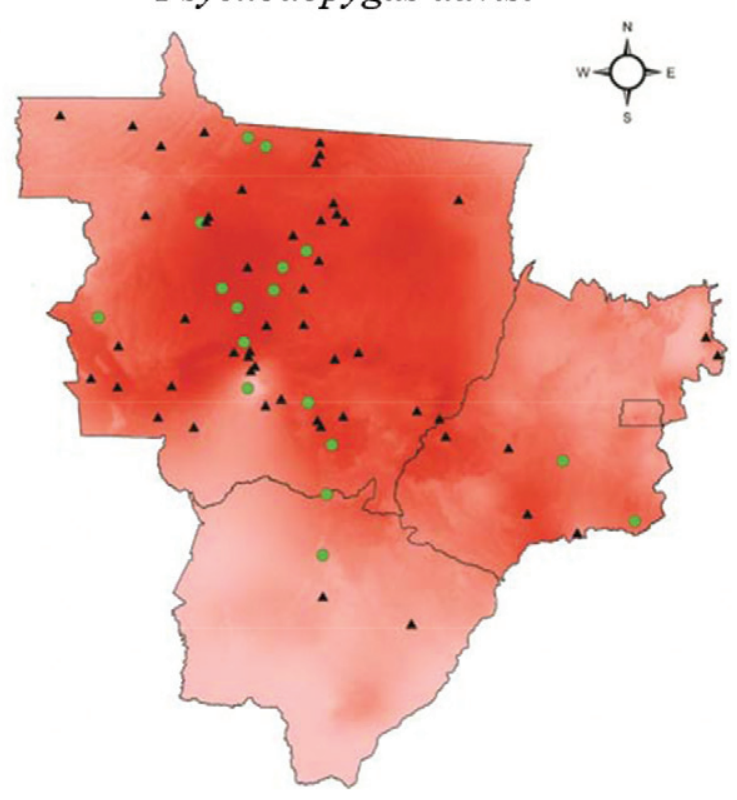

- Training points

- Test points
Climatic suitability

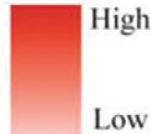

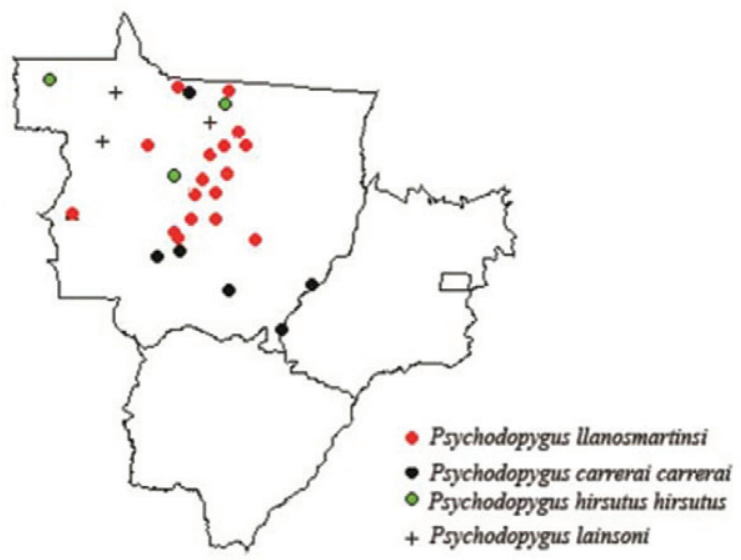

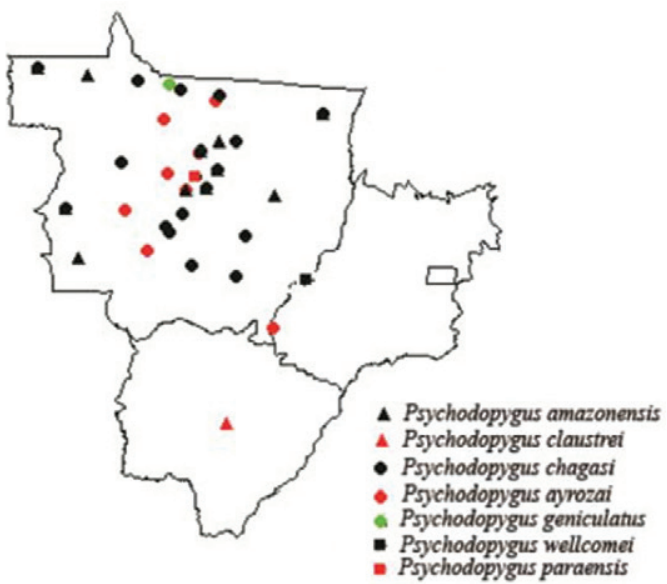




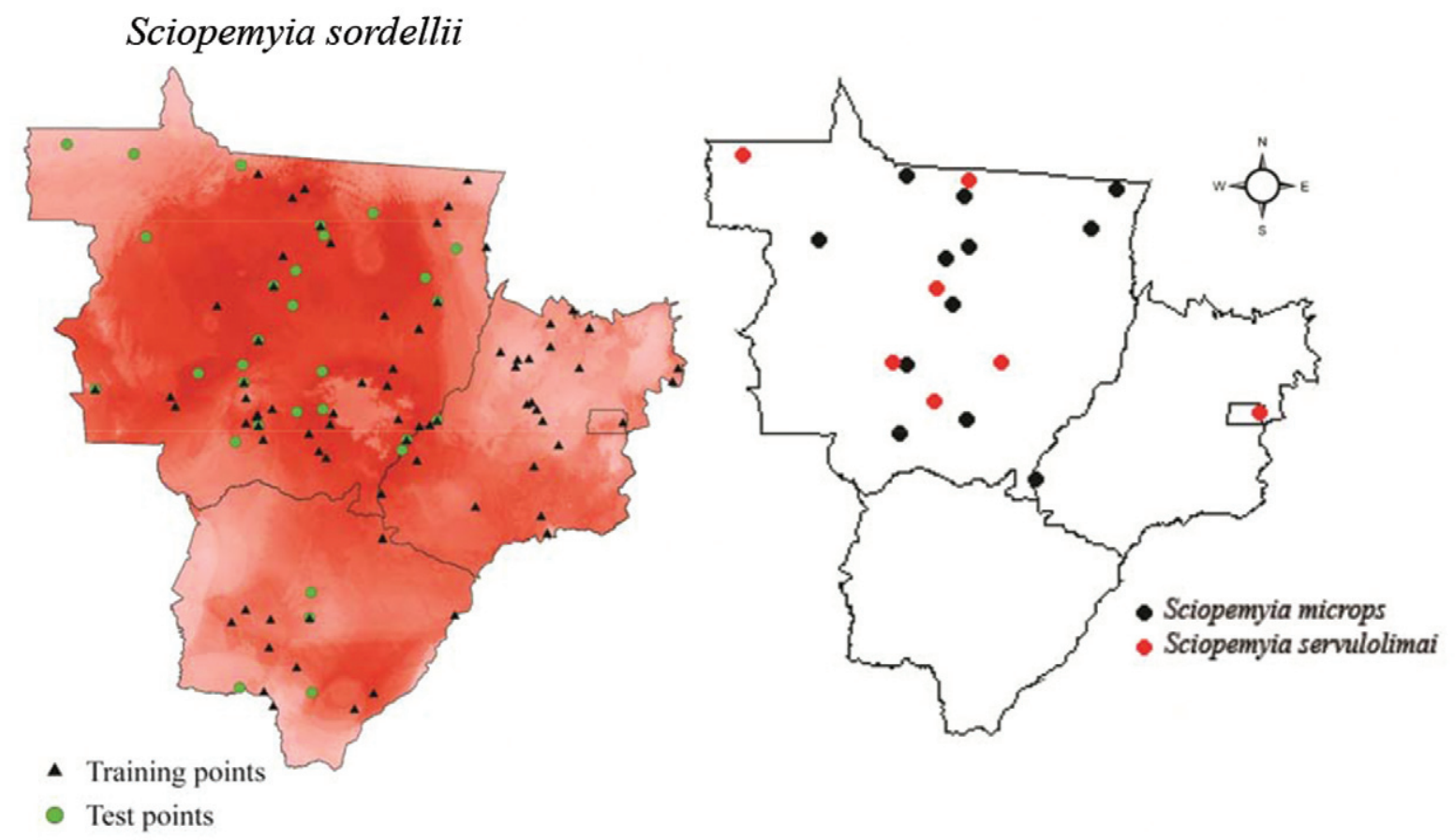

\section{Climatic suitability}

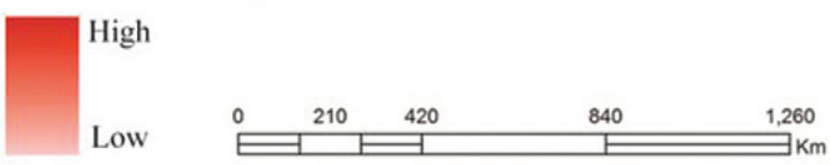




\section{Trychopygomyia dasypodogeton}
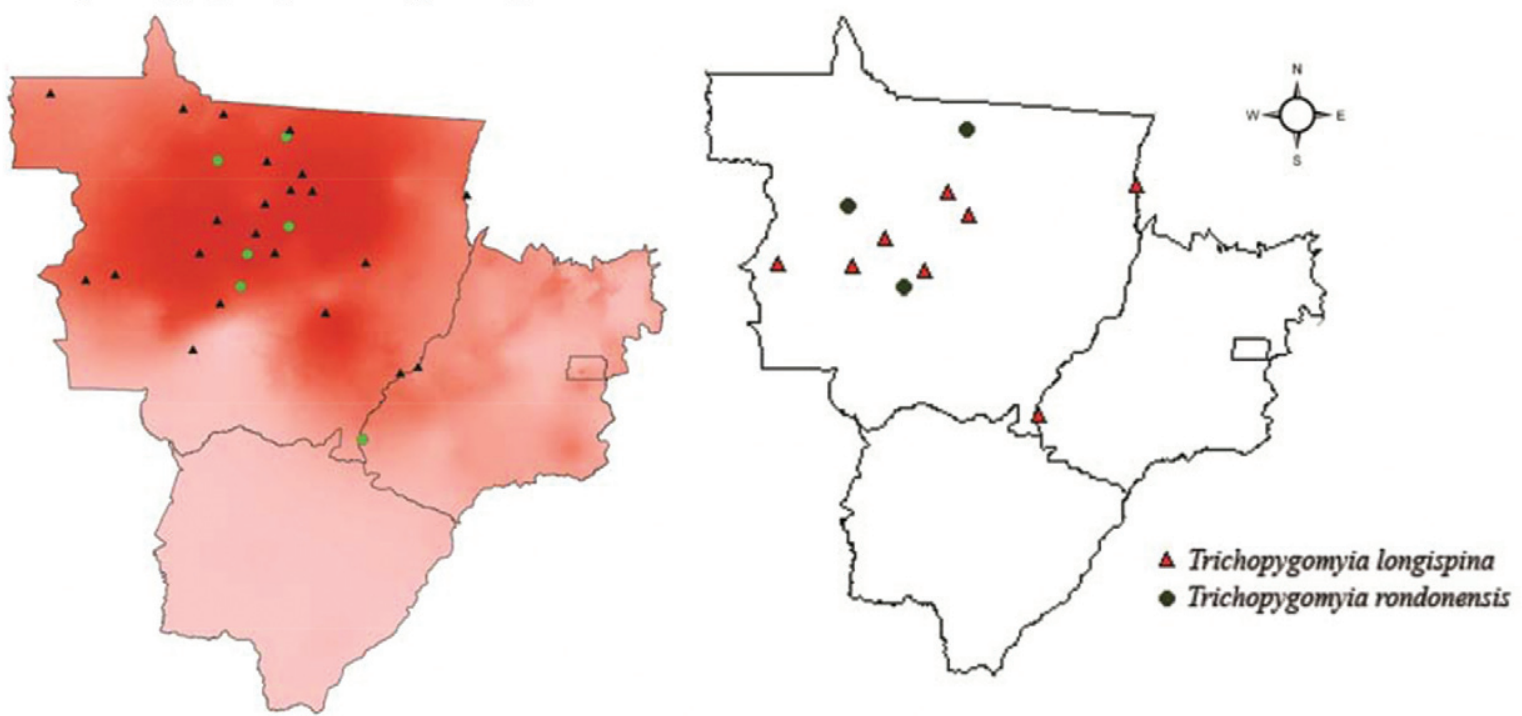

A Training points

- Test points

\section{Climatic suitability}

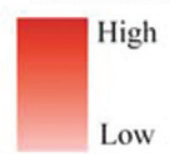

\begin{tabular}{lllll}
0 & 210 & 420 & 840 & 1,260 \\
\hline & & &
\end{tabular} 
- Training points

- Test points

Climatic suitability

High

Low

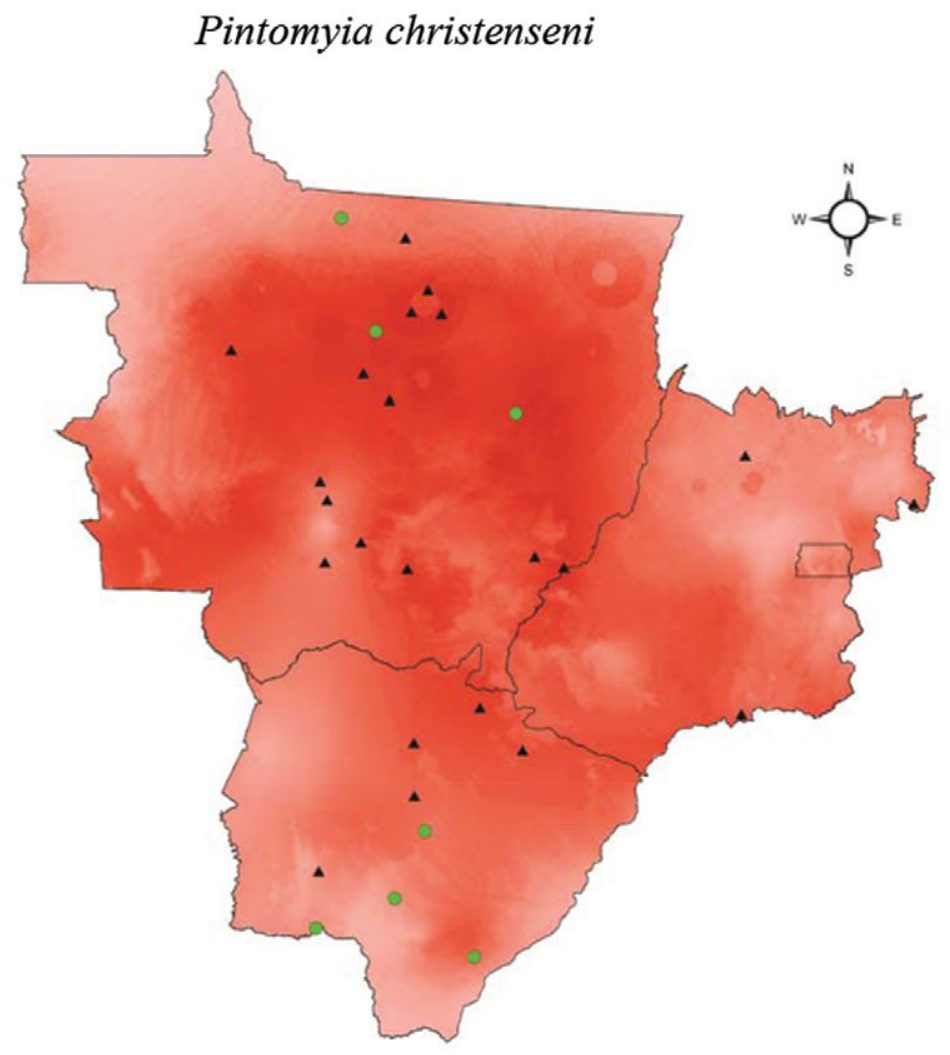

$\begin{array}{lll}187.5 & 375 & 750\end{array}$

$1,125 \quad 1,500$
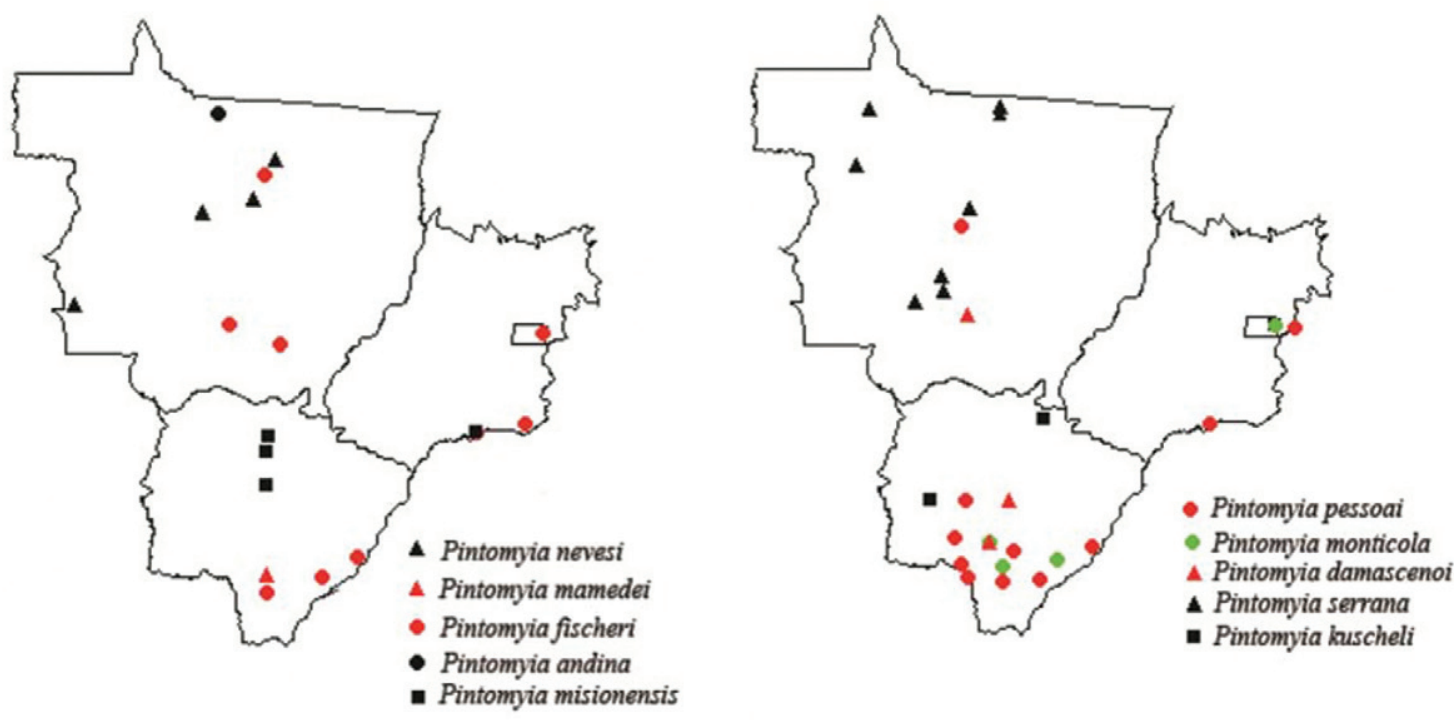
Viannamyia

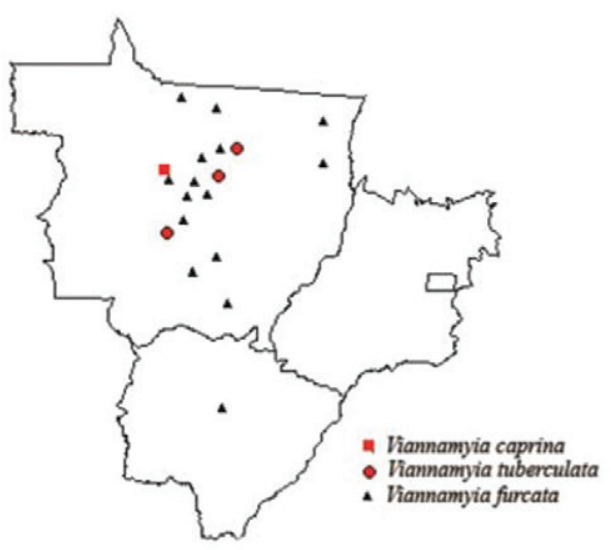

Martinsmyia

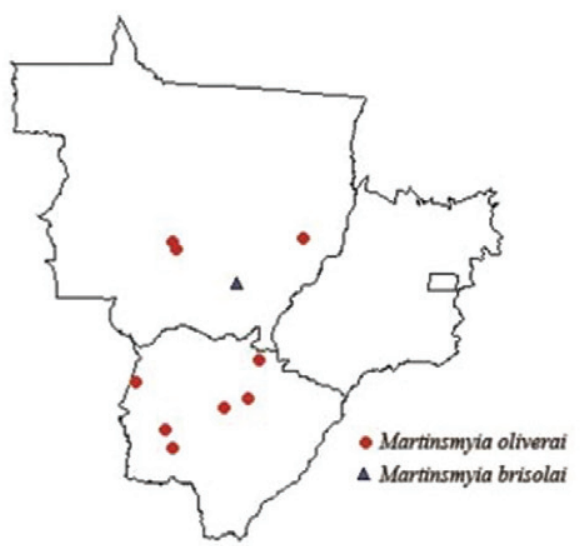

\section{Bichromomyia}

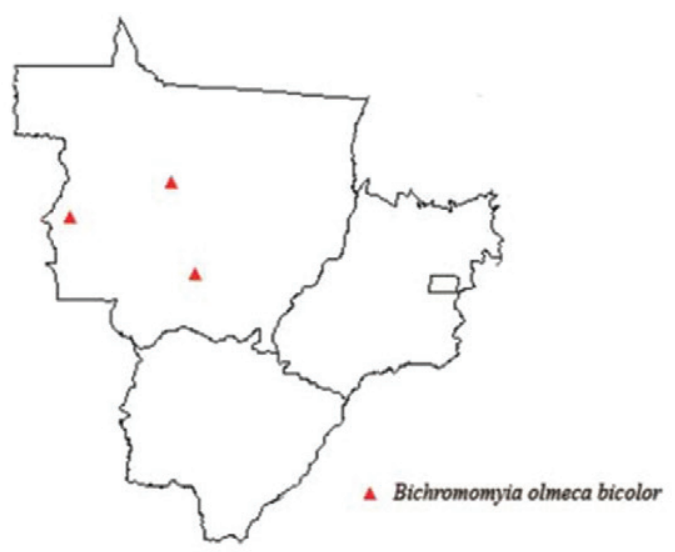

Nyssomyia

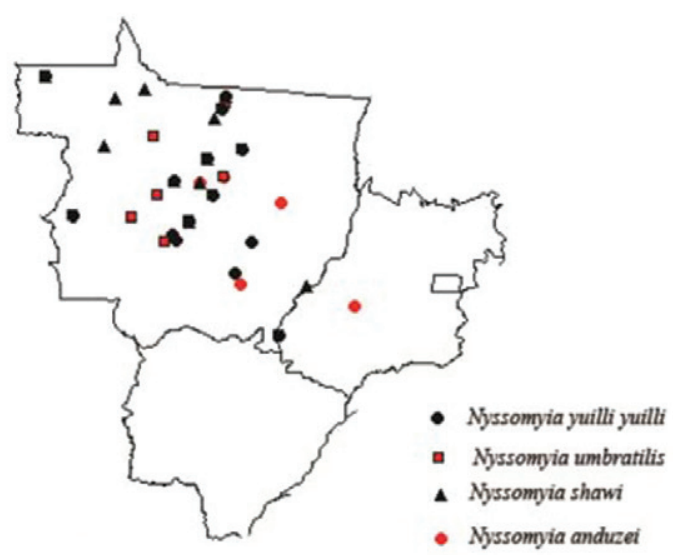

Trichophoromyia

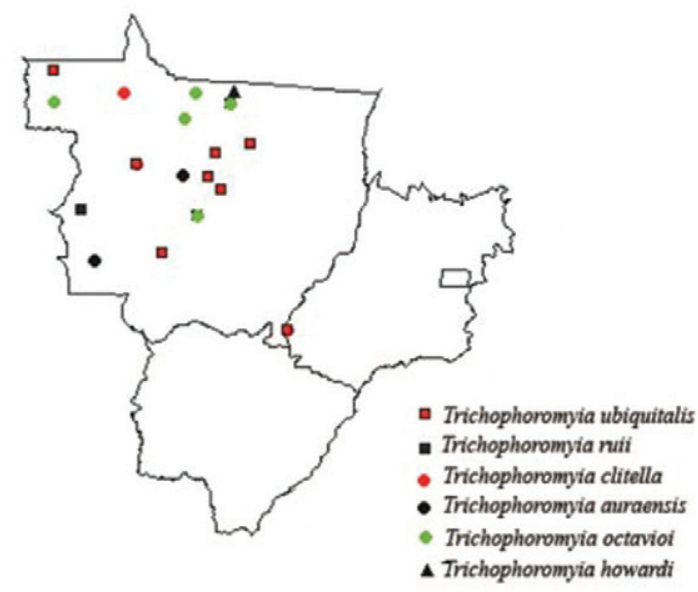




\section{Expapillata}

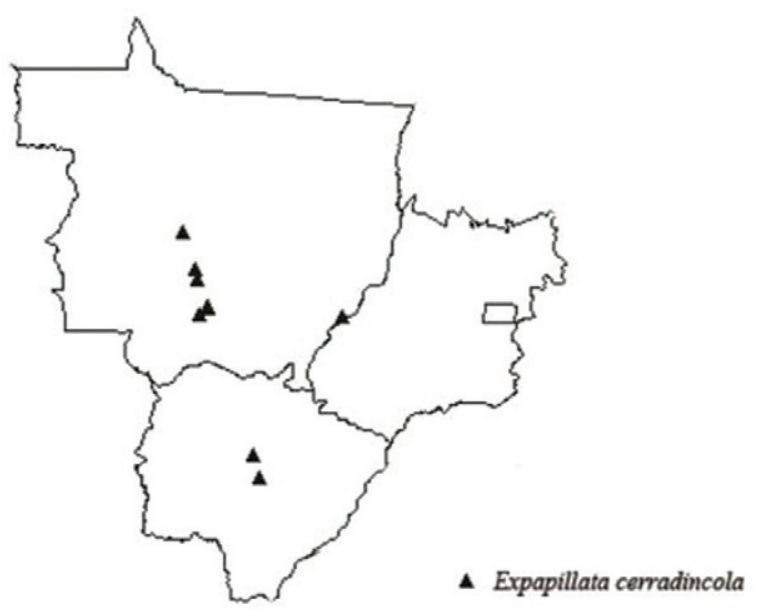

\section{Lutzomyia}

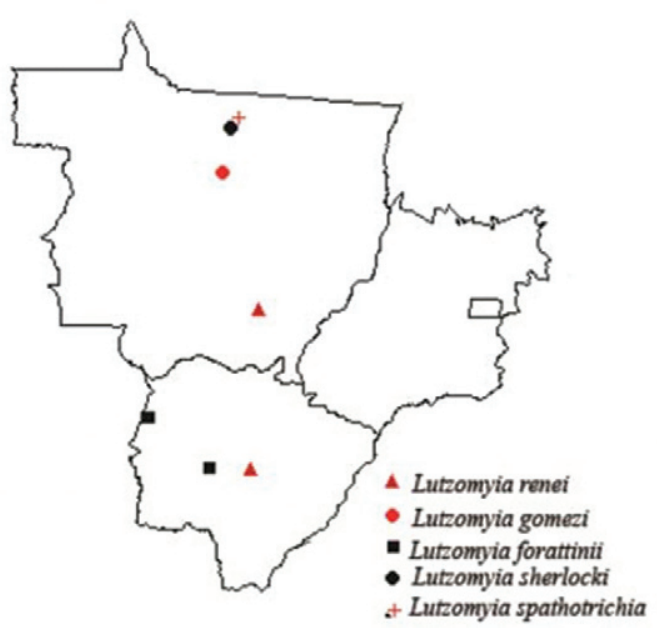

Pressatia

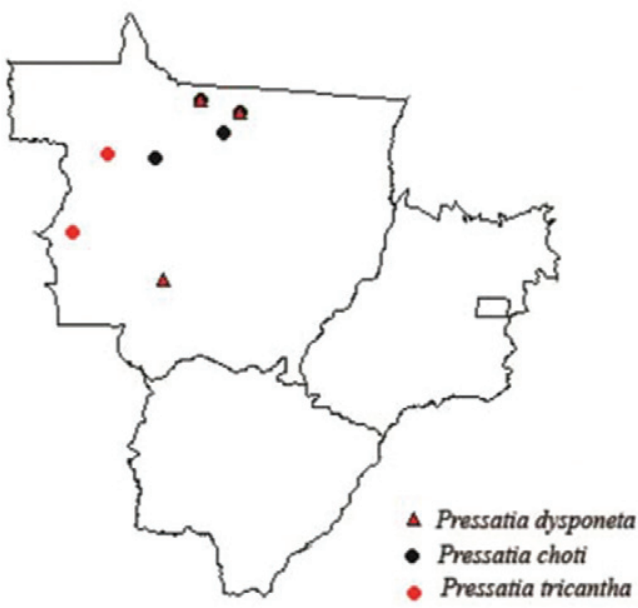

- Pressatia tricantha

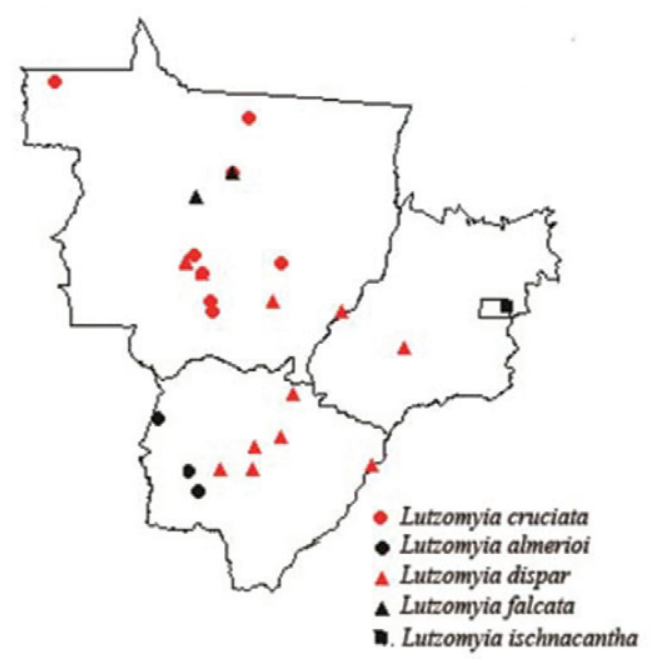

Geographic distribution maps of other phlebotomine species in Central-West Brazil. 
TABLE

Geographic coordinates used to produce ecological niche models of phlebotomine species in Central-West Brazil

\begin{tabular}{|c|c|c|c|c|}
\hline Sandfly species & State code & Municipalities & Longitude & Latitude \\
\hline Bichromomyia flaviscutellata & MT & Barra dos Bugres & -57.18 & -15.08 \\
\hline Bichromomyia flaviscutellata & MT & Varzea Grande & -56.13 & -15.65 \\
\hline Bichromomyia flaviscutellata & MT & Sapezal & -57.20 & -14.97 \\
\hline Bichromomyia flaviscutellata & MT & Brasnorte & -57.98 & -12.15 \\
\hline Bichromomyia flaviscutellata & MT & Nova Mutum & -56.08 & -13.83 \\
\hline Bichromomyia flaviscutellata & MT & Barra do Garças & -52.26 & -15.89 \\
\hline Bichromomyia flaviscutellata & MT & Água Boa & -52.16 & -14.05 \\
\hline Bichromomyia flaviscutellata & MT & Alta Floresta & -56.09 & -9.88 \\
\hline Bichromomyia flaviscutellata & MT & Apiacás & -57.45 & -9.54 \\
\hline Bichromomyia flaviscutellata & MT & Aripuanã & -60.63 & -9.17 \\
\hline Bichromomyia flaviscutellata & MT & Barão do Melgaço & -55.97 & -16.19 \\
\hline Bichromomyia flaviscutellata & MT & Campo Novo do Parecis & -57.88 & -13.67 \\
\hline Bichromomyia flaviscutellata & MT & Campo Verde & -55.16 & -15.54 \\
\hline Bichromomyia flaviscutellata & MT & Canabrava do Norte & -51.83 & -11.03 \\
\hline Bichromomyia flaviscutellata & MT & Chapada dos Guimarães & -55.75 & -15.46 \\
\hline Bichromomyia flaviscutellata & MT & Cláudia & -54.88 & -11.49 \\
\hline Bichromomyia flaviscutellata & MT & Colíder & -55.45 & -10.81 \\
\hline Bichromomyia flaviscutellata & MT & Colniza & -59.03 & -9.40 \\
\hline Bichromomyia flaviscutellata & MT & Comodoro & -59.79 & -13.66 \\
\hline Bichromomyia flaviscutellata & MT & Cuiabá & -56.10 & -15.60 \\
\hline Bichromomyia flaviscutellata & MT & Diamantino & -56.45 & -14.41 \\
\hline Bichromomyia flaviscutellata & MT & Feliz Natal & -54.92 & -12.39 \\
\hline Bichromomyia flaviscutellata & MT & Gaúcha do Norte & -53.08 & -13.24 \\
\hline Bichromomyia flaviscutellata & MT & General Carneiro & -52.76 & -15.71 \\
\hline Bichromomyia flaviscutellata & MT & Guarantã do Norte & -54.90 & -9.78 \\
\hline Bichromomyia flaviscutellata & MT & Itiquira & -54.14 & -17.21 \\
\hline Bichromomyia flaviscutellata & MT & Lucas do Rio Verde & -55.91 & -13.05 \\
\hline Bichromomyia flaviscutellata & MT & Mercelândia & -54.60 & -11.11 \\
\hline Bichromomyia flaviscutellata & MT & Nobres & -56.33 & -14.73 \\
\hline Bichromomyia flaviscutellata & MT & Nova Brasilândia & -54.57 & -14.57 \\
\hline Bichromomyia flaviscutellata & MT & Nova Lacerda & -59.35 & -14.28 \\
\hline Bichromomyia flaviscutellata & MT & Nova Maringá & -57.06 & -13.01 \\
\hline Bichromomyia flaviscutellata & MT & Nova Ubiratã & -55.26 & -13.01 \\
\hline Bichromomyia flaviscutellata & MT & Novo Horizonte do Norte & -57.35 & -11.41 \\
\hline Bichromomyia flaviscutellata & MT & Pontal do Araguaia & -52.01 & -15.85 \\
\hline Bichromomyia flaviscutellata & MT & Pontes de Lacerda & -59.37 & -15.20 \\
\hline Bichromomyia flaviscutellata & MT & Porto Alegre do Norte & -51.63 & -10.87 \\
\hline Bichromomyia flaviscutellata & MT & Poxoréo & -54.38 & -15.83 \\
\hline Bichromomyia flaviscutellata & MT & Primavera do Leste & -54.30 & -15.56 \\
\hline Bichromomyia flaviscutellata & MT & Ribeirão Cascalheira & -51.82 & -12.92 \\
\hline Bichromomyia flaviscutellata & MT & Rondonópolis & -54.64 & -16.47 \\
\hline Bichromomyia flaviscutellata & MT & Rosário Oeste & -56.43 & -14.84 \\
\hline Bichromomyia flaviscutellata & MT & Santa Rita do Trivelato & -55.26 & -13.81 \\
\hline Bichromomyia flaviscutellata & MT & São Felix do Araguaia & -50.67 & -11.61 \\
\hline Bichromomyia flaviscutellata & MT & Sapezal & -57.20 & -14.97 \\
\hline Bichromomyia flaviscutellata & MT & Sinop & -55.49 & -11.83 \\
\hline Bichromomyia flaviscutellata & MT & Sorriso & -55.71 & -12.55 \\
\hline Bichromomyia flaviscutellata & MT & Tangará da Serra & -57.50 & -14.62 \\
\hline Bichromomyia flaviscutellata & MT & União do Sul & -54.36 & -11.52 \\
\hline Bichromomyia flaviscutellata & MS & Aquidauana & -55.79 & -20.47 \\
\hline Bichromomyia flaviscutellata & MS & Bela Vista & -56.52 & -22.11 \\
\hline Bichromomyia flaviscutellata & MS & Campo Grande & -54.65 & -20.44 \\
\hline
\end{tabular}




\begin{tabular}{|c|c|c|c|c|}
\hline Sandfly species & State code & Municipalities & Longitude & Latitude \\
\hline Bichromomyia flaviscutellata & MS & São Gabriel do Oeste & -54.57 & -19.40 \\
\hline Bichromomyia flaviscutellata & MS & Santa Rita do Pardo & -52.83 & -21.30 \\
\hline Bichromomyia flaviscutellata & MS & Bonito & -56.47 & -21.13 \\
\hline Bichromomyia flaviscutellata & MS & Pedro Gomes & -54.55 & -18.10 \\
\hline Bichromomyia flaviscutellata & MS & Rio Verde de Mato Grosso & -54.84 & -18.92 \\
\hline Bichromomyia flaviscutellata & MS & Sonora & -54.75 & -17.57 \\
\hline Bichromomyia flaviscutellata & MS & Três Lagoas & -51.68 & -20.75 \\
\hline Bichromomyia flaviscutellata & $\mathrm{GO}$ & Cavalcante & -47.50 & -13.80 \\
\hline Bichromomyia flaviscutellata & $\mathrm{GO}$ & Porangatu & -49.15 & -13.44 \\
\hline Bichromomyia flaviscutellata & DF & Brasília & -47.42 & -15.78 \\
\hline Brumptomyia avellari & MS & Água Clara & -52.87 & -20.44 \\
\hline Brumptomyia avellari & MS & Alcinópolis & -53.70 & -18.31 \\
\hline Brumptomyia avellari & MS & Anastácio & -55.81 & -20.48 \\
\hline Brumptomyia avellari & MS & Aquidauana & -55.79 & -20.47 \\
\hline Brumptomyia avellari & MS & Bela Vista & -56.52 & -22.11 \\
\hline Brumptomyia avellari & MS & Bonito & -56.47 & -21.13 \\
\hline Brumptomyia avellari & MS & Brasilândia & -52.04 & -21.26 \\
\hline Brumptomyia avellari & MS & Camapuã & -54.04 & -19.53 \\
\hline Brumptomyia avellari & MS & Campo Grande & -54.65 & -20.44 \\
\hline Brumptomyia avellari & MS & Corumbá & -57.65 & -19.01 \\
\hline Brumptomyia avellari & MS & Corguinho & -54.83 & -19.83 \\
\hline Brumptomyia avellari & MS & Figueirão & -53.38 & -18.4 \\
\hline Brumptomyia avellari & MS & Fátima do Sul & -54.51 & -22.37 \\
\hline Brumptomyia avellari & MS & Ribas do Rio Pardo & -53.76 & -20.44 \\
\hline Brumptomyia avellari & MS & Maracajú & -55.17 & -21.61 \\
\hline Brumptomyia avellari & MS & Navirí & -54.22 & -23.13 \\
\hline Brumptomyia avellari & MS & Nioaque & -55.83 & -21.14 \\
\hline Brumptomyia avellari & MS & Nhecolânia & -57.07 & -17.27 \\
\hline Brumptomyia avellari & MS & Nova Andradina & -53.34 & -22.23 \\
\hline Brumptomyia avellari & MS & Novo Horizonte do Sul & -53.80 & -22.61 \\
\hline Brumptomyia avellari & MS & Rochedo & -54.89 & -19.95 \\
\hline Brumptomyia avellari & MS & São Gabriel do Oeste & -54.57 & -19.40 \\
\hline Brumptomyia avellari & MS & Dourados & -54.81 & -22.22 \\
\hline Brumptomyia avellari & MS & Guia Lopes da Laguna & -56.11 & -21.46 \\
\hline Brumptomyia avellari & MS & Santa Rita do Pardo & -52.83 & -21.30 \\
\hline Brumptomyia avellari & MS & Bonito & -56.47 & -21.13 \\
\hline Brumptomyia avellari & MS & Dois Irmãos do Buriti & -55.30 & -20.68 \\
\hline Brumptomyia avellari & MS & Paranhos & -55.43 & -23.89 \\
\hline Brumptomyia avellari & MS & Rio Verde de Mato Grosso & -54.84 & -18.92 \\
\hline Brumptomyia avellari & MS & Três Lagoas & -51.68 & -20.75 \\
\hline Brumptomyia avellari & $\mathrm{GO}$ & Itumbiara & -49.21 & -18.42 \\
\hline Brumptomyia avellari & $\mathrm{GO}$ & Sítio d' Abadia & -46.25 & -14.80 \\
\hline Brumptomyia avellari & DF & Brasília & -47.42 & -15.78 \\
\hline Brumptomyia brumpti & MT & Peixoto de Azevedo & -54.98 & -10.22 \\
\hline Brumptomyia brumpti & MT & Várzea Grande & -56.13 & -15.65 \\
\hline Brumptomyia brumpti & MT & Nova Mutum & -56.08 & -13.83 \\
\hline Brumptomyia brumpti & MT & Cuiabá & -56.10 & -15.60 \\
\hline Brumptomyia brumpti & MT & Barra do Garças & -52.26 & -15.89 \\
\hline Brumptomyia brumpti & MT & Água Boa & -52.16 & -14.05 \\
\hline Brumptomyia brumpti & MT & Alta Floresta & -56.09 & -9.88 \\
\hline Brumptomyia brumpti & MT & Alto do Paraguai & -56.48 & -14.51 \\
\hline Brumptomyia brumpti & MT & Araguainha & -53.08 & -16.82 \\
\hline Brumptomyia brumpti & MT & Arenápolis & -56.82 & -14.43 \\
\hline
\end{tabular}




\begin{tabular}{|c|c|c|c|c|}
\hline Sandfly species & State code & Municipalities & Longitude & Latitude \\
\hline Brumptomyia brumpti & MT & Barra dos Bugres & -57.18 & -15.08 \\
\hline Brumptomyia brumpti & MT & Barra do Garças & -52.26 & -15.89 \\
\hline Brumptomyia brumpti & MT & Chapada dos Guimarães & -55.75 & -15.46 \\
\hline Brumptomyia brumpti & MT & Cláudia & -54.88 & -11.49 \\
\hline Brumptomyia brumpti & MT & Denise & -57.05 & -14.73 \\
\hline Brumptomyia brumpti & MT & Diamantino & -56.45 & -14.41 \\
\hline Brumptomyia brumpti & MT & Itiquira & -54.14 & -17.21 \\
\hline Brumptomyia brumpti & MT & Jaciara & -54.95 & -15.92 \\
\hline Brumptomyia brumpti & MT & Novo Horizonte do Norte & -57.35 & -11.41 \\
\hline Brumptomyia brumpti & MT & Novo São Joaquim & -53.02 & -14.90 \\
\hline Brumptomyia brumpti & MT & Poconé & -56.62 & -16.26 \\
\hline Brumptomyia brumpti & MT & Pontal do Araguaia & -52.00 & -15.84 \\
\hline Brumptomyia brumpti & MT & Porto Alegre do Norte & -51.63 & -10.87 \\
\hline Brumptomyia brumpti & MT & Porto dos Gaúchos & -57.41 & -11.52 \\
\hline Brumptomyia brumpti & MT & Rondonópolis & -54.64 & -16.47 \\
\hline Brumptomyia brumpti & MT & Rosário Oeste & -56.43 & -14.84 \\
\hline Brumptomyia brumpti & MT & Santo Antônio do Leverger & -56.08 & -15.87 \\
\hline Brumptomyia brumpti & MT & São José dos 4 Marcos & -58.17 & -15.17 \\
\hline Brumptomyia brumpti & MT & Sinop & -55.49 & -11.83 \\
\hline Brumptomyia brumpti & MT & Tangará da Serra & -57.50 & -14.62 \\
\hline Brumptomyia brumpti & MT & Itanhangá & -56.64 & -12.23 \\
\hline Brumptomyia brumpti & MS & Água Clara & -52.87 & -20.44 \\
\hline Brumptomyia brumpti & MS & Alcinópolis & -53.70 & -18.31 \\
\hline Brumptomyia brumpti & MS & Antonio João & -55.95 & -22.19 \\
\hline Brumptomyia brumpti & MS & Bela Vista & -56.52 & -22.11 \\
\hline Brumptomyia brumpti & MS & Bonito & -56.47 & -21.13 \\
\hline Brumptomyia brumpti & MS & Bataguassu & -52.42 & -21.71 \\
\hline Brumptomyia brumpti & MS & Bodoquena & -56.72 & -20.54 \\
\hline Brumptomyia brumpti & MS & Brasilândia & -52.04 & -21.26 \\
\hline Brumptomyia brumpti & MS & Corumbá & -57.65 & -19.01 \\
\hline Brumptomyia brumpti & MS & Campo Grande & -54.65 & -20.44 \\
\hline Brumptomyia brumpti & MS & Fátima do Sul & -54.51 & -22.37 \\
\hline Brumptomyia brumpti & MS & Nova Andradina & -53.34 & -22.23 \\
\hline Brumptomyia brumpti & MS & São Gabriel do Oeste & -54.57 & -19.40 \\
\hline Brumptomyia brumpti & MS & Terenos & -54.86 & -20.44 \\
\hline Brumptomyia brumpti & MS & Costa Rica & -53.13 & -18.54 \\
\hline Brumptomyia brumpti & MS & Dourados & -54.81 & -22.22 \\
\hline Brumptomyia brumpti & MS & Guia Lopes da Laguna & -56.11 & -21.46 \\
\hline Brumptomyia brumpti & MS & Inocência & -51.93 & -19.73 \\
\hline Brumptomyia brumpti & MS & Jardim & -56.14 & -21.48 \\
\hline Brumptomyia brumpti & MS & Nioaque & -55.83 & -21.13 \\
\hline Brumptomyia brumpti & MS & Santa Rita do Pardo & -52.83 & -21.30 \\
\hline Brumptomyia brumpti & MS & Bonito & -41.27 & -11.97 \\
\hline Brumptomyia brumpti & MS & Rio Verde de Mato Grosso & -54.84 & -18.92 \\
\hline Brumptomyia brumpti & MS & Três Lagoas & -51.68 & -20.75 \\
\hline Evandromyia carmelinoi & MT & Várzea Grande & -56.13 & -15.65 \\
\hline Evandromyia carmelinoi & MT & Cuiabá & -56.10 & -15.60 \\
\hline Evandromyia carmelinoi & MT & Água Boa & -52.16 & -14.05 \\
\hline Evandromyia carmelinoi & MT & Alta Floresta & -56.09 & -9.88 \\
\hline Evandromyia carmelinoi & MT & Alto Araguaia & -53.22 & -17.31 \\
\hline Evandromyia carmelinoi & MT & Alto da Boa Vista & -51.38 & -11.66 \\
\hline Evandromyia carmelinoi & MT & Alto do Paraguai & -56.48 & -14.51 \\
\hline Evandromyia carmelinoi & MT & Araguaina & -51.83 & -15.73 \\
\hline
\end{tabular}




\begin{tabular}{|c|c|c|c|c|}
\hline Sandfly species & State code & Municipalities & Longitude & Latitude \\
\hline Evandromyia carmelinoi & MT & Araguainha & -53.08 & -16.82 \\
\hline Evandromyia carmelinoi & MT & Arenápolis & -56.82 & -14.43 \\
\hline Evandromyia carmelinoi & MT & Aripuanã & -60.63 & -9.17 \\
\hline Evandromyia carmelinoi & MT & Barão do Melgaço & -55.97 & -16.19 \\
\hline Evandromyia carmelinoi & MT & Barra dos Bugres & -57.18 & -15.08 \\
\hline Evandromyia carmelinoi & MT & Barra do Garças & -52.26 & -15.89 \\
\hline Evandromyia carmelinoi & MT & Brasnorte & -57.98 & -12.15 \\
\hline Evandromyia carmelinoi & MT & Campinápolis & -52.88 & -14.50 \\
\hline Evandromyia carmelinoi & MT & Campo Novo do Parecis & -57.88 & -13.67 \\
\hline Evandromyia carmelinoi & MT & Campo Verde & -55.16 & -15.54 \\
\hline Evandromyia carmelinoi & MT & Canabrava do Norte & -51.83 & -11.03 \\
\hline Evandromyia carmelinoi & MT & Canarana & -52.27 & -13.55 \\
\hline Evandromyia carmelinoi & MT & Chapada dos Guimarães & -55.75 & -15.46 \\
\hline Evandromyia carmelinoi & MT & Claúdia & -54.88 & -11.49 \\
\hline Evandromyia carmelinoi & MT & Colíder & -55.45 & -10.81 \\
\hline Evandromyia carmelinoi & MT & Comodoro & -59.79 & -13.66 \\
\hline Evandromyia carmelinoi & MT & Confresa & -51.56 & -10.64 \\
\hline Evandromyia carmelinoi & MT & Diamantino & -56.45 & -14.41 \\
\hline Evandromyia carmelinoi & MT & Feliz Natal & -54.92 & -12.39 \\
\hline Evandromyia carmelinoi & MT & Gaúcha do Norte & -53.08 & -13.24 \\
\hline Evandromyia carmelinoi & MT & Gerneral Carneiro & -52.76 & -15.71 \\
\hline Evandromyia carmelinoi & MT & Guarantã do Norte & -54.90 & -9.78 \\
\hline Evandromyia carmelinoi & MT & Jaciara & -54.95 & -15.92 \\
\hline Evandromyia carmelinoi & MT & Nossa S. do Livramento & -56.37 & -15.80 \\
\hline Evandromyia carmelinoi & MT & Nova Brasilândia & -54.57 & -14.57 \\
\hline Evandromyia carmelinoi & MT & Nova Canaã do Norte & -57.35 & -11.41 \\
\hline Evandromyia carmelinoi & MT & Nova Marilândia & -56.57 & -14.21 \\
\hline Evandromyia carmelinoi & MT & Nova Maringá & -57.06 & -13.01 \\
\hline Evandromyia carmelinoi & MT & Nova Mutum & -56.08 & -13.83 \\
\hline Evandromyia carmelinoi & MT & Nova Ubiratã & -55.26 & -13.01 \\
\hline Evandromyia carmelinoi & MT & Novo Horizonte do Norte & -57.35 & -11.41 \\
\hline Evandromyia carmelinoi & MT & Novo São Joaquim & -53.02 & -14.90 \\
\hline Evandromyia carmelinoi & MT & Paratinga & -54.05 & -14.43 \\
\hline Evandromyia carmelinoi & MT & Peixoto de Azevedo & -54.98 & -10.22 \\
\hline Evandromyia carmelinoi & MT & Planalto da Serra & -55.27 & -10.44 \\
\hline Evandromyia carmelinoi & MT & Poconé & -56.62 & -16.26 \\
\hline Evandromyia carmelinoi & MT & Pontal do Araguaia & -52.00 & -15.84 \\
\hline Evandromyia carmelinoi & MT & Ponte Branca & -56.78 & -16.08 \\
\hline Evandromyia carmelinoi & MT & Porto Alegre do Norte & -51.63 & -10.87 \\
\hline Evandromyia carmelinoi & MT & Porto dos Gaúchos & -57.41 & -11.52 \\
\hline Evandromyia carmelinoi & MT & Porto Estrela & -57.22 & -15.32 \\
\hline Evandromyia carmelinoi & MT & Poxoréo & -54.38 & -15.83 \\
\hline Evandromyia carmelinoi & MT & Primavera do Leste & -54.30 & -15.56 \\
\hline Evandromyia carmelinoi & MT & Querência & -52.11 & -12.35 \\
\hline Evandromyia carmelinoi & MT & Rondonópolis & -54.64 & -16.47 \\
\hline Evandromyia carmelinoi & MT & Rosário Oeste & -56.43 & -14.84 \\
\hline Evandromyia carmelinoi & MT & Santa Rita do Trivelato & -55.26 & -13.81 \\
\hline Evandromyia carmelinoi & MT & São José do Rio Claro & -56.72 & -13.44 \\
\hline Evandromyia carmelinoi & MT & Sinop & -55.49 & -11.83 \\
\hline Evandromyia carmelinoi & MT & Sorriso & -55.71 & -12.55 \\
\hline Evandromyia carmelinoi & MT & Taboporã & -56.62 & -10.80 \\
\hline Evandromyia carmelinoi & MT & Tangará da Serra & -57.50 & -14.62 \\
\hline Evandromyia carmelinoi & MT & Tapurah & -56.50 & -12.53 \\
\hline
\end{tabular}




\begin{tabular}{|c|c|c|c|c|}
\hline Sandfly species & State code & Municipalities & Longitude & Latitude \\
\hline Evandromyia carmelinoi & MT & União do Sul & -54.36 & -11.52 \\
\hline Evandromyia carmelinoi & MT & Vera & -55.19 & -12.18 \\
\hline Evandromyia carmelinoi & MT & Vila Rica & -51.11 & -10.01 \\
\hline Evandromyia carmelinoi & MT & Itanhangá & -56.64 & -12.23 \\
\hline Evandromyia carmelinoi & MT & Ipiranga do Norte & -56.05 & -15.35 \\
\hline Evandromyia carmelinoi & MS & Água Clara & -52.87 & -20.44 \\
\hline Evandromyia carmelinoi & MS & Antonio João & -55.95 & -22.19 \\
\hline Evandromyia carmelinoi & MS & Aparecida do Taboado & -51.09 & -20.09 \\
\hline Evandromyia carmelinoi & MS & Cassilândia & -51.73 & -19.11 \\
\hline Evandromyia carmelinoi & MS & Paranaíba & -51.19 & -19.68 \\
\hline Evandromyia carmelinoi & MS & Três Lagoas & -51.68 & -20.75 \\
\hline Evandromyia carmelinoi & MS & Novo horizonte do Sul & -53.80 & -22.61 \\
\hline Evandromyia carmelinoi & MS & Nova Andradina & -53.34 & -22.23 \\
\hline Evandromyia carmelinoi & MS & Paraíso das Águas & -52.97 & -19.05 \\
\hline Evandromyia carmelinoi & MS & Rio Verde de Mato Grosso & -54.84 & -18.92 \\
\hline Evandromyia evandroi & MT & Varzea Grande & -56.13 & -15.65 \\
\hline Evandromyia evandroi & MT & Cuiabá & -56.10 & -15.60 \\
\hline Evandromyia evandroi & MT & Barra do Garças & -52.26 & -15.89 \\
\hline Evandromyia evandroi & MT & Acorizal & -56.37 & -15.20 \\
\hline Evandromyia evandroi & MT & Água Boa & -52.16 & -14.05 \\
\hline Evandromyia evandroi & MT & Alta Floresta & -56.09 & -9.88 \\
\hline Evandromyia evandroi & MT & Alto da Boa Vista & -51.38 & -11.66 \\
\hline Evandromyia evandroi & MT & Alto do Paraguai & -56.48 & -14.51 \\
\hline Evandromyia evandroi & MT & Araguaina & -51.83 & -15.73 \\
\hline Evandromyia evandroi & MT & Aripuanã & -60.63 & -9.17 \\
\hline Evandromyia evandroi & MT & Barão do Melgaço & -55.97 & -16.19 \\
\hline Evandromyia evandroi & MT & Barra dos Bugres & -57.18 & -15.08 \\
\hline Evandromyia evandroi & MT & Brasnorte & -57.98 & -12.15 \\
\hline Evandromyia evandroi & MT & Campinápolis & -57.68 & -16.07 \\
\hline Evandromyia evandroi & MT & Campo Novo do Parecis & -57.88 & -13.67 \\
\hline Evandromyia evandroi & MT & Campo Verde & -55.16 & -15.54 \\
\hline Evandromyia evandroi & MT & Canabrava do Norte & -51.83 & -11.03 \\
\hline Evandromyia evandroi & MT & Canarana & -52.27 & -13.55 \\
\hline Evandromyia evandroi & MT & Carolina & -55.83 & -9.96 \\
\hline Evandromyia evandroi & MT & Chapada dos Guimarães & -55.75 & -15.46 \\
\hline Evandromyia evandroi & MT & Claúdia & -54.88 & -11.49 \\
\hline Evandromyia evandroi & MT & Colíder & -55.45 & -10.81 \\
\hline Evandromyia evandroi & MT & Colniza & -59.03 & -9.40 \\
\hline Evandromyia evandroi & MT & Confresa & -51.56 & -10.64 \\
\hline Evandromyia evandroi & MT & Denise & -57.05 & -14.73 \\
\hline Evandromyia evandroi & MT & Diamantino & -56.45 & -14.41 \\
\hline Evandromyia evandroi & MT & Dom Aquino & -54.55 & -15.48 \\
\hline Evandromyia evandroi & MT & Feliz Natal & -54.92 & -12.39 \\
\hline Evandromyia evandroi & MT & Gaúcha do Norte & -53.08 & -13.24 \\
\hline Evandromyia evandroi & MT & Gerneral Carneiro & -52.76 & -15.71 \\
\hline Evandromyia evandroi & MT & Guiratinga & -53.74 & -16.33 \\
\hline Evandromyia evandroi & MT & Itiquira & -54.14 & -17.21 \\
\hline Evandromyia evandroi & MT & Jaciara & -54.95 & -15.92 \\
\hline Evandromyia evandroi & MT & Jangada & -56.49 & -15.24 \\
\hline Evandromyia evandroi & MT & Juscimeira & -54.88 & -16.05 \\
\hline Evandromyia evandroi & MT & Lucas do Rio Verde & -55.91 & -13.05 \\
\hline Evandromyia evandroi & MT & Luciara & -50.57 & -10.75 \\
\hline Evandromyia evandroi & MT & Mercelândia & -54.60 & -11.11 \\
\hline
\end{tabular}




\begin{tabular}{|c|c|c|c|c|}
\hline Sandfly species & State code & Municipalities & Longitude & Latitude \\
\hline Evandromyia evandroi & MT & Matupá & -54.90 & -10.05 \\
\hline Evandromyia evandroi & MT & Mirassol d' Oeste & -58.05 & -15.40 \\
\hline Evandromyia evandroi & MT & Nobres & -56.33 & -14.73 \\
\hline Evandromyia evandroi & MT & Nossa S. do Livramento & -56.37 & -15.80 \\
\hline Evandromyia evandroi & MT & Nova Brasilândia & -54.57 & -14.57 \\
\hline Evandromyia evandroi & MT & Nova Maringá & -57.06 & -13.01 \\
\hline Evandromyia evandroi & MT & Nova Mutum & -56.08 & -13.83 \\
\hline Evandromyia evandroi & MT & Nova Santana Helena & -53.35 & -10.82 \\
\hline Evandromyia evandroi & MT & Nova Ubiratã & -55.26 & -13.01 \\
\hline Evandromyia evandroi & MT & Novo Horizonte do Norte & -57.35 & -11.41 \\
\hline Evandromyia evandroi & MT & Novo São Joaquim & -53.02 & -14.90 \\
\hline Evandromyia evandroi & MT & Paratinga & -54.05 & -14.43 \\
\hline Evandromyia evandroi & MT & Peixoto de Azevedo & -54.98 & -10.22 \\
\hline Evandromyia evandroi & MT & Planalto da Serra & -55.27 & -10.44 \\
\hline Evandromyia evandroi & MT & Pontal do Araguaia & -52.01 & -15.85 \\
\hline Evandromyia evandroi & MT & Ponte Branca & -56.78 & -16.08 \\
\hline Evandromyia evandroi & MT & Porto Alegre do Norte & -51.63 & -10.87 \\
\hline Evandromyia evandroi & MT & Porto Estrela & -57.22 & -15.32 \\
\hline Evandromyia evandroi & MT & Poxoréo & -54.38 & -15.83 \\
\hline Evandromyia evandroi & MT & Primavera do Leste & -54.30 & -15.56 \\
\hline Evandromyia evandroi & MT & Querência & -52.11 & -12.35 \\
\hline Evandromyia evandroi & MT & Ribeirão Cascalheira & -51.82 & -12.92 \\
\hline Evandromyia evandroi & MT & Rondonópolis & -54.64 & -16.47 \\
\hline Evandromyia evandroi & MT & Rosário Oeste & -56.43 & -14.84 \\
\hline Evandromyia evandroi & MT & Santa Rita do Trivelato & -55.26 & -13.81 \\
\hline Evandromyia evandroi & MT & Santo Antônio do Leverger & -56.08 & -15.87 \\
\hline Evandromyia evandroi & MT & São Felix do Araguaia & -50.67 & -11.61 \\
\hline Evandromyia evandroi & MT & São José do Povo & -54.25 & -16.46 \\
\hline Evandromyia evandroi & MT & São José dos 4 Marcos & -58.17 & -15.17 \\
\hline Evandromyia evandroi & MT & Sapezal & -57.20 & -14.97 \\
\hline Evandromyia evandroi & MT & Serra da Nova Dourado & -51.58 & -11.97 \\
\hline Evandromyia evandroi & MT & Sinop & -55.49 & -11.83 \\
\hline Evandromyia evandroi & MT & Sorriso & -55.71 & -12.55 \\
\hline Evandromyia evandroi & MT & Teorixoréu & -52.56 & -16.20 \\
\hline Evandromyia evandroi & MT & União do Sul & -54.36 & -11.52 \\
\hline Evandromyia evandroi & $\mathrm{GO}$ & Bom Jardim & -52.12 & -16.28 \\
\hline Evandromyia evandroi & $\mathrm{GO}$ & Campos Verdes & -49.66 & -14.26 \\
\hline Evandromyia evandroi & GO & Campestre de Goiás & -49.69 & -16.730 \\
\hline Evandromyia evandroi & $\mathrm{GO}$ & Mambaí & -46.11 & -14.49 \\
\hline Evandromyia evandroi & GO & Mutunópolis & -49.28 & -13.73 \\
\hline Evandromyia evandroi & $\mathrm{GO}$ & Niquelândia & -48.46 & -14.47 \\
\hline Evandromyia evandroi & $\mathrm{GO}$ & Nova Crixás & -50.33 & -14.10 \\
\hline Evandromyia evandroi & $\mathrm{GO}$ & Rio Verde & -50.93 & -17.80 \\
\hline Evandromyia evandroi & GO & São Domingos & -46.32 & -13.40 \\
\hline Evandromyia evandroi & $\mathrm{GO}$ & São João d'Aliança & -47.79 & -14.88 \\
\hline Evandromyia evandroi & $\mathrm{GO}$ & São Miguel da Araguaina & -50.22 & -13.32 \\
\hline Evandromyia evandroi & GO & Silvânia & 48.61 & -16.66 \\
\hline Evandromyia evandroi & GO & Uruaçu & -49.14 & -14.52 \\
\hline Evandromyia evandroi & $\mathrm{GO}$ & Amaro Leite & -49.15 & -13.97 \\
\hline Evandromyia evandroi & DF & Brasília & -47.42 & -15.78 \\
\hline Evandromyia evandroi & MS & Aparecida do Taboado & -51.09 & -20.09 \\
\hline Evandromyia evandroi & MS & Aquidauana & -55.79 & -20.47 \\
\hline Evandromyia evandroi & MS & Bela Vista & -56.52 & -22.11 \\
\hline
\end{tabular}




\begin{tabular}{|c|c|c|c|c|}
\hline Sandfly species & State code & Municipalities & Longitude & Latitude \\
\hline Evandromyia evandroi & MS & Bodoquena & -56.72 & -20.54 \\
\hline Evandromyia evandroi & MS & Coxim & -54.76 & -18.51 \\
\hline Evandromyia evandroi & MS & Rio Negro & -54.99 & -19.45 \\
\hline Evandromyia evandroi & MS & Selvíria & -51.42 & -20.37 \\
\hline Evandromyia evandroi & MS & Três Lagoas & -51.68 & -20.75 \\
\hline Evandromyia evandroi & MS & Jardim & -56.14 & -21.48 \\
\hline Evandromyia evandroi & MS & Três Lagoas & -51.68 & -20.75 \\
\hline Evandromyia lenti & MT & Varzea Grande & -56.13 & -15.65 \\
\hline Evandromyia lenti & MT & Nova Mutum & -56.08 & -13.83 \\
\hline Evandromyia lenti & MT & Cuiabá & -56.10 & -15.60 \\
\hline Evandromyia lenti & MT & Barra do Garças & -52.26 & -15.89 \\
\hline Evandromyia lenti & MT & Acorizal & -56.37 & -15.20 \\
\hline Evandromyia lenti & MT & Água Boa & -52.16 & -14.05 \\
\hline Evandromyia lenti & MT & Alta Floresta & -56.09 & -9.88 \\
\hline Evandromyia lenti & MT & Alto Araguaia & -53.22 & -17.31 \\
\hline Evandromyia lenti & MT & Alto da Boa Vista & -51.38 & -11.66 \\
\hline Evandromyia lenti & MT & Alto do Paraguai & -56.48 & -14.51 \\
\hline Evandromyia lenti & MT & Araguaina & -51.83 & -15.73 \\
\hline Evandromyia lenti & MT & Araguainha & -53.08 & -16.82 \\
\hline Evandromyia lenti & MT & Arenápolis & -56.82 & -14.43 \\
\hline Evandromyia lenti & MT & Barão do Melgaço & -55.97 & -16.19 \\
\hline Evandromyia lenti & MT & Barra dos Bugres & -57.18 & -15.08 \\
\hline Evandromyia lenti & MT & Brasnorte & -57.98 & -12.15 \\
\hline Evandromyia lenti & MT & Campo Novo do Parecis & -57.88 & -13.67 \\
\hline Evandromyia lenti & MT & Campo Verde & -55.16 & -15.54 \\
\hline Evandromyia lenti & MT & Canabrava do Norte & -51.83 & -11.03 \\
\hline Evandromyia lenti & MT & Canarana & -52.27 & -13.55 \\
\hline Evandromyia lenti & MT & Chapada dos Guimarães & -55.75 & -15.46 \\
\hline Evandromyia lenti & MT & Claúdia & -54.88 & -11.49 \\
\hline Evandromyia lenti & MT & Colíder & -55.45 & -10.81 \\
\hline Evandromyia lenti & MT & Comodoro & -59.79 & -13.66 \\
\hline Evandromyia lenti & MT & Confresa & -51.56 & -10.64 \\
\hline Evandromyia lenti & MT & Denise & -57.05 & -14.73 \\
\hline Evandromyia lenti & MT & Diamantino & -56.45 & -14.41 \\
\hline Evandromyia lenti & MT & Feliz Natal & -54.92 & -12.39 \\
\hline Evandromyia lenti & MT & Gaúcha do Norte & -53.08 & -13.24 \\
\hline Evandromyia lenti & MT & Gerneral Carneiro & -52.76 & -15.71 \\
\hline Evandromyia lenti & MT & Guarantã do Norte & -54.90 & -9.78 \\
\hline Evandromyia lenti & MT & Guiratinga & -53.74 & -16.33 \\
\hline Evandromyia lenti & MT & Itiquira & -54.14 & -17.21 \\
\hline Evandromyia lenti & MT & Jaciara & -54.95 & -15.92 \\
\hline Evandromyia lenti & MT & Jangada & -56.49 & -15.24 \\
\hline Evandromyia lenti & MT & Juscimeira & -54.88 & -16.05 \\
\hline Evandromyia lenti & MT & Lucas do Rio Verde & -55.91 & -13.05 \\
\hline Evandromyia lenti & MT & Luciara & -50.57 & -10.75 \\
\hline Evandromyia lenti & MT & Mercelândia & -54.60 & -11.11 \\
\hline Evandromyia lenti & MT & Matupá & -54.90 & -10.05 \\
\hline Evandromyia lenti & MT & Nobres & -56.33 & -14.73 \\
\hline Evandromyia lenti & MT & Nossa S. do Livramento & -56.37 & -15.80 \\
\hline Evandromyia lenti & MT & Nova Brasilândia & -54.57 & -14.57 \\
\hline Evandromyia lenti & MT & Nova Marilândia & -56.57 & -14.21 \\
\hline Evandromyia lenti & MT & Nova Maringá & -57.06 & -13.01 \\
\hline Evandromyia lenti & MT & Nova Santana Helena & -53.35 & -10.82 \\
\hline
\end{tabular}




\begin{tabular}{|c|c|c|c|c|}
\hline Sandfly species & State code & Municipalities & Longitude & Latitude \\
\hline Evandromyia lenti & MT & Nova Ubiratã & -55.26 & -13.01 \\
\hline Evandromyia lenti & MT & Novo Horizonte do Norte & -57.35 & -11.41 \\
\hline Evandromyia lenti & MT & Novo São Joaquim & -53.02 & -14.90 \\
\hline Evandromyia lenti & MT & Paranaíta & -56.48 & -9.67 \\
\hline Evandromyia lenti & MT & Paratinga & -54.05 & -14.43 \\
\hline Evandromyia lenti & MT & Peixoto de Azevedo & -54.98 & -10.22 \\
\hline Evandromyia lenti & MT & Planalto da Serra & -55.27 & -10.44 \\
\hline Evandromyia lenti & MT & Poconé & -56.62 & -16.26 \\
\hline Evandromyia lenti & MT & Pontal do Araguaia & -52.01 & -15.85 \\
\hline Evandromyia lenti & MT & Porto Alegre do Norte & -51.63 & -10.87 \\
\hline Evandromyia lenti & MT & Porto Esperidião & -58.47 & -15.85 \\
\hline Evandromyia lenti & MT & Poxoréo & -54.38 & -15.83 \\
\hline Evandromyia lenti & MT & Primavera do Leste & -54.30 & -15.56 \\
\hline Evandromyia lenti & MT & Ribeirão Cascalheira & -51.82 & -12.92 \\
\hline Evandromyia lenti & MT & Ribeirãozinho & -52.67 & -16.45 \\
\hline Evandromyia lenti & MT & Rondonópolis & -54.64 & -16.47 \\
\hline Evandromyia lenti & MT & Rosário Oeste & -56.43 & -14.84 \\
\hline Evandromyia lenti & MT & Santa Carmem & -54.52 & -11.35 \\
\hline Evandromyia lenti & MT & Santa Rita do Trivelato & -55.26 & -13.81 \\
\hline Evandromyia lenti & MT & Santo Antônio do Leverger & -56.08 & -15.87 \\
\hline Evandromyia lenti & MT & São Felix do Araguaia & -50.67 & -11.61 \\
\hline Evandromyia lenti & MT & São José dos 4 Marcos & -58.17 & -15.17 \\
\hline Evandromyia lenti & MT & Sapezal & -57.20 & -14.97 \\
\hline Evandromyia lenti & MT & Serra da Nova Dourado & -51.58 & -11.97 \\
\hline Evandromyia lenti & MT & Sinop & -55.49 & -11.83 \\
\hline Evandromyia lenti & MT & Sorriso & -55.71 & -12.55 \\
\hline Evandromyia lenti & MT & Taboporã & -56.62 & -10.80 \\
\hline Evandromyia lenti & MT & Tangará da Serra & -57.50 & -14.62 \\
\hline Evandromyia lenti & MT & Tapurah & -56.50 & -12.53 \\
\hline Evandromyia lenti & MT & Terra nova do Norte & -55.23 & -10.51 \\
\hline Evandromyia lenti & MT & Teorixoréu & -52.56 & -16.20 \\
\hline Evandromyia lenti & MT & União do Sul & -54.36 & -11.52 \\
\hline Evandromyia lenti & MT & Itanhangá & -56.64 & -12.23 \\
\hline Evandromyia lenti & MT & Ipiranga do Norte & -56.05 & -15.35 \\
\hline Evandromyia lenti & $\mathrm{GO}$ & Aragarças & -50.63 & -15.09 \\
\hline Evandromyia lenti & GO & Araguapaz & -50.63 & -15.09 \\
\hline Evandromyia lenti & $\mathrm{GO}$ & Bela Vista & -48.95 & -16.97 \\
\hline Evandromyia lenti & GO & Bom Jardim & -52.12 & -16.28 \\
\hline Evandromyia lenti & $\mathrm{GO}$ & Campos Verdes & -49.66 & -14.26 \\
\hline Evandromyia lenti & GO & Campestre de Goiás & -49.41 & -16.45 \\
\hline Evandromyia lenti & GO & Colinas do Sul & -48.08 & -14.15 \\
\hline Evandromyia lenti & $\mathrm{GO}$ & Cumari & -48.15 & -18.26 \\
\hline Evandromyia lenti & $\mathrm{GO}$ & Catalão & -47.95 & -18.17 \\
\hline Evandromyia lenti & GO & Crixás & -49.97 & -14.45 \\
\hline Evandromyia lenti & $\mathrm{GO}$ & Goiás & -50.14 & -15.93 \\
\hline Evandromyia lenti & $\mathrm{GO}$ & Goiatuba & -49.37 & -18.02 \\
\hline Evandromyia lenti & $\mathrm{GO}$ & Itumbiara & -49.22 & -18.42 \\
\hline Evandromyia lenti & GO & Guapó & -49.53 & -16.83 \\
\hline Evandromyia lenti & GO & Mambaí & -46.11 & -14.49 \\
\hline Evandromyia lenti & $\mathrm{GO}$ & Montividiu do Norte & -48.61 & -13.11 \\
\hline Evandromyia lenti & $\mathrm{GO}$ & Mutunópolis & -49.28 & -13.73 \\
\hline Evandromyia lenti & $\mathrm{GO}$ & Mundo Novo & -40.47 & -11.86 \\
\hline Evandromyia lenti & $\mathrm{GO}$ & Minaçu & -48.22 & -13.53 \\
\hline
\end{tabular}




\begin{tabular}{|c|c|c|c|c|}
\hline Sandfly species & State code & Municipalities & Longitude & Latitude \\
\hline Evandromyia lenti & $\mathrm{GO}$ & Niquelândia & -48.46 & -14.47 \\
\hline Evandromyia lenti & $\mathrm{GO}$ & Nova Crixás & -50.33 & -14.10 \\
\hline Evandromyia lenti & $\mathrm{GO}$ & Porangatu & -49.15 & -13.44 \\
\hline Evandromyia lenti & $\mathrm{GO}$ & Rio Verde & -50.93 & -17.80 \\
\hline Evandromyia lenti & $\mathrm{GO}$ & São Domingos & -46.32 & -13.40 \\
\hline Evandromyia lenti & $\mathrm{GO}$ & São Miguel da Araguaina & -50.22 & -13.32 \\
\hline Evandromyia lenti & $\mathrm{GO}$ & Silvânia & 48.61 & -16.66 \\
\hline Evandromyia lenti & $\mathrm{GO}$ & Uriapuru & -49.92 & -14.29 \\
\hline Evandromyia lenti & $\mathrm{GO}$ & Uruaçu & -49.14 & -14.52 \\
\hline Evandromyia lenti & $\mathrm{GO}$ & Sítio d'Abadia & -46.25 & -14.80 \\
\hline Evandromyia lenti & $\mathrm{GO}$ & Amaro Leite & -49.15 & -13.97 \\
\hline Evandromyia lenti & DF & Brasília & -47.42 & -15.78 \\
\hline Evandromyia lenti & MS & Água Clara & -52.87 & -20.44 \\
\hline Evandromyia lenti & MS & Alcinópolis & -53.71 & -18.32 \\
\hline Evandromyia lenti & MS & Anaurilândia & -52.43 & -22.10 \\
\hline Evandromyia lenti & MS & Anastácio & -55.81 & -20.48 \\
\hline Evandromyia lenti & MS & Antonio João & -55.95 & -22.19 \\
\hline Evandromyia lenti & MS & Aquidauana & -55.79 & -20.47 \\
\hline Evandromyia lenti & MS & Bela Vista & -56.52 & -22.11 \\
\hline Evandromyia lenti & MS & Bodoquena & -56.72 & -20.54 \\
\hline Evandromyia lenti & MS & Campo Grande & -54.65 & -20.44 \\
\hline Evandromyia lenti & MS & Canmapuã & -54.04 & -19.53 \\
\hline Evandromyia lenti & MS & Corguinho & -54.83 & -19.83 \\
\hline Evandromyia lenti & MS & Corumbá & -57.65 & -19.01 \\
\hline Evandromyia lenti & MS & Figueirão & -53.38 & -18.4 \\
\hline Evandromyia lenti & MS & Coxim & -54.76 & -18.51 \\
\hline Evandromyia lenti & MS & Ponta Porã & -55.73 & -22.54 \\
\hline Evandromyia lenti & MS & Rio Negro & -54.99 & -19.45 \\
\hline Evandromyia lenti & MS & Rochedo & -54.89 & -19.95 \\
\hline Evandromyia lenti & MS & São Gabriel do Oeste & -54.57 & -19.40 \\
\hline Evandromyia lenti & MS & Tacurú & -55.02 & -23.63 \\
\hline Evandromyia lenti & MS & Terenos & -54.86 & -20.44 \\
\hline Evandromyia lenti & MS & Três Lagoas & -51.68 & -20.75 \\
\hline Evandromyia lenti & MS & Dourados & -54.81 & -22.22 \\
\hline Evandromyia lenti & MS & Miranda & -56.38 & -20.24 \\
\hline Evandromyia lenti & MS & Guia Lopes da Laguna & -56.11 & -21.46 \\
\hline Evandromyia lenti & MS & Nova Andradina & -53.34 & -22.23 \\
\hline Evandromyia lenti & MS & Novo Horizonte & -53.80 & -22.61 \\
\hline Evandromyia lenti & MS & Nioaque & -55.83 & -21.14 \\
\hline Evandromyia lenti & MS & Santa Rita do Pardo & -52.83 & -21.30 \\
\hline Evandromyia lenti & MS & Bonito & -56.47 & -21.13 \\
\hline Evandromyia lenti & MS & Maracajú & -55.17 & -21.61 \\
\hline Evandromyia lenti & MS & Pedro Gomes & -54.55 & -18.10 \\
\hline Evandromyia lenti & MS & Rio Verde de Mato Grosso & -54.84 & -18.92 \\
\hline Evandromyia saulensis & MT & Barra dos Bugres & -57.18 & -15.08 \\
\hline Evandromyia saulensis & MT & Rosário d' Oeste & -56.43 & -14.84 \\
\hline Evandromyia saulensis & MT & Barra do Garças & -52.26 & -15.89 \\
\hline Evandromyia saulensis & MT & Varzea Grande & -56.13 & -15.65 \\
\hline Evandromyia saulensis & MT & Nova Mutum & -56.08 & -13.83 \\
\hline Evandromyia saulensis & MT & Cuiabá & -56.10 & -15.60 \\
\hline Evandromyia saulensis & MT & Alta Floresta & -56.09 & -9.88 \\
\hline Evandromyia saulensis & MT & Alto do Paraguai & -56.48 & -14.51 \\
\hline Evandromyia saulensis & MT & Alto Taquarí & -53.16 & -17.49 \\
\hline
\end{tabular}




\begin{tabular}{|c|c|c|c|c|}
\hline Sandfly species & State code & Municipalities & Longitude & Latitude \\
\hline Evandromyia saulensis & MT & Aripuanã & -60.63 & -9.17 \\
\hline Evandromyia saulensis & MT & Barão do Melgaço & -55.97 & -16.19 \\
\hline Evandromyia saulensis & MT & Brasnorte & -57.98 & -12.15 \\
\hline Evandromyia saulensis & MT & Campinápolis & -52.88 & -14.50 \\
\hline Evandromyia saulensis & MT & Campo Verde & -55.16 & -15.54 \\
\hline Evandromyia saulensis & MT & Canarana & -52.27 & -13.55 \\
\hline Evandromyia saulensis & MT & Carolina & -55.83 & -9.96 \\
\hline Evandromyia saulensis & MT & Chapada dos Guimarães & -55.75 & -15.46 \\
\hline Evandromyia saulensis & MT & Colíder & -55.45 & -10.81 \\
\hline Evandromyia saulensis & MT & Colniza & -59.03 & -9.40 \\
\hline Evandromyia saulensis & MT & Comodoro & -59.79 & -13.66 \\
\hline Evandromyia saulensis & MT & Diamantino & -59.79 & -13.66 \\
\hline Evandromyia saulensis & MT & Dom Aquino & -54.55 & -15.48 \\
\hline Evandromyia saulensis & MT & Feliz Natal & -54.92 & -12.39 \\
\hline Evandromyia saulensis & MT & Gaúcha do Norte & -53.08 & -13.24 \\
\hline Evandromyia saulensis & MT & Guarantã do Norte & -54.90 & -9.78 \\
\hline Evandromyia saulensis & MT & Itiquira & -56.64 & -12.23 \\
\hline Evandromyia saulensis & MT & Jaciara & -54.95 & -15.92 \\
\hline Evandromyia saulensis & MT & Jangada & -56.49 & -15.24 \\
\hline Evandromyia saulensis & MT & Juara & -54.95 & -15.92 \\
\hline Evandromyia saulensis & MT & Juína & -58.74 & -11.38 \\
\hline Evandromyia saulensis & MT & Lucas do Rio Verde & -55.91 & -13.05 \\
\hline Evandromyia saulensis & MT & Luciara & -50.57 & -10.75 \\
\hline Evandromyia saulensis & MT & Matupá & -54.90 & -10.05 \\
\hline Evandromyia saulensis & MT & Nobres & -56.33 & -14.73 \\
\hline Evandromyia saulensis & MT & Nortelândia & -56.80 & -14.42 \\
\hline Evandromyia saulensis & MT & Nossa S. do Livramento & -56.37 & -15.80 \\
\hline Evandromyia saulensis & MT & Nova Brasilândia & -54.57 & -14.57 \\
\hline Evandromyia saulensis & MT & Nova Canaã do Norte & -55.95 & -10.55 \\
\hline Evandromyia saulensis & MT & Novo Horizonte do Norte & -57.35 & -11.41 \\
\hline Evandromyia saulensis & MT & Paranaíta & -56.48 & -9.67 \\
\hline Evandromyia saulensis & MT & Paratinga & -54.05 & -14.43 \\
\hline Evandromyia saulensis & MT & Peixoto de Azevedo & -54.98 & -10.22 \\
\hline Evandromyia saulensis & MT & Planalto da Serra & -55.27 & -10.44 \\
\hline Evandromyia saulensis & MT & Pontes de Lacerda & -59.37 & -15.20 \\
\hline Evandromyia saulensis & MT & Porto Alegre do Norte & -51.63 & -10.87 \\
\hline Evandromyia saulensis & MT & Porto dos Gaúchos & -57.41 & -11.52 \\
\hline Evandromyia saulensis & MT & Porto Estrela & -57.22 & -15.32 \\
\hline Evandromyia saulensis & MT & Poxoréo & -54.38 & -15.83 \\
\hline Evandromyia saulensis & MT & Primavera do Leste & -54.30 & -15.56 \\
\hline Evandromyia saulensis & MT & Rondonópolis & -54.64 & -16.47 \\
\hline Evandromyia saulensis & MT & Santo Antônio do Leverger & -56.08 & -15.87 \\
\hline Evandromyia saulensis & MT & São Felix do Araguaia & -50.67 & -11.61 \\
\hline Evandromyia saulensis & MT & São José dos 4 Marcos & -58.17 & -15.17 \\
\hline Evandromyia saulensis & MT & Sinop & -55.49 & -11.83 \\
\hline Evandromyia saulensis & MT & Sorriso & -55.71 & -12.55 \\
\hline Evandromyia saulensis & MT & Terra nova do Norte & -55.23 & -10.51 \\
\hline Evandromyia saulensis & MT & União do Sul & -54.36 & -11.52 \\
\hline Evandromyia saulensis & $\mathrm{GO}$ & Itumbiara & -49.22 & -18.42 \\
\hline Evandromyia saulensis & $\mathrm{GO}$ & Catalão & -47.95 & -18.17 \\
\hline Evandromyia saulensis & DF & Brasília & -47.42 & -15.78 \\
\hline Evandromyia saulensis & MS & Alcinópolis & -53.70 & -18.31 \\
\hline Evandromyia saulensis & MS & Coxim & -54.76 & -18.51 \\
\hline
\end{tabular}




\begin{tabular}{|c|c|c|c|c|}
\hline Sandfly species & State code & Municipalities & Longitude & Latitude \\
\hline Evandromyia termitophila & MT & Varzea Grande & -56.13 & -15.65 \\
\hline Evandromyia termitophila & MT & Cuiabá & -56.10 & -15.60 \\
\hline Evandromyia termitophila & MT & Barra do Garças & -52.26 & -15.89 \\
\hline Evandromyia termitophila & MT & Acorizal & -56.37 & -15.20 \\
\hline Evandromyia termitophila & MT & Água Boa & -52.16 & -14.05 \\
\hline Evandromyia termitophila & MT & Alta Floresta & -56.09 & -9.88 \\
\hline Evandromyia termitophila & MT & Alto do Paraguai & -56.48 & -14.51 \\
\hline Evandromyia termitophila & MT & Araguaina & -51.83 & -15.73 \\
\hline Evandromyia termitophila & MT & Arenápolis & -56.82 & -14.43 \\
\hline Evandromyia termitophila & MT & Aripuanã & -60.63 & -9.17 \\
\hline Evandromyia termitophila & MT & Barão do Melgaço & -55.97 & -16.19 \\
\hline Evandromyia termitophila & MT & Brasnorte & -57.98 & -12.15 \\
\hline Evandromyia termitophila & MT & Campinápolis & -52.88 & -14.50 \\
\hline Evandromyia termitophila & MT & Campo Novo do Parecis & -57.88 & -13.67 \\
\hline Evandromyia termitophila & MT & Campo Verde & -55.16 & -15.54 \\
\hline Evandromyia termitophila & MT & Canabrava do Norte & -51.83 & -11.03 \\
\hline Evandromyia termitophila & MT & Canarana & -52.27 & -13.55 \\
\hline Evandromyia termitophila & MT & Carolina & -55.83 & -9.96 \\
\hline Evandromyia termitophila & MT & Chapada dos Guimarães & -55.75 & -15.46 \\
\hline Evandromyia termitophila & MT & Claúdia & -54.88 & -11.49 \\
\hline Evandromyia termitophila & MT & Colíder & -55.45 & -10.81 \\
\hline Evandromyia termitophila & MT & Colniza & -59.03 & -9.40 \\
\hline Evandromyia termitophila & MT & Comodoro & -59.79 & -13.66 \\
\hline Evandromyia termitophila & MT & Confresa & -51.56 & -10.64 \\
\hline Evandromyia termitophila & MT & Diamantino & -56.45 & -14.41 \\
\hline Evandromyia termitophila & MT & Dom Aquino & -54.55 & -15.48 \\
\hline Evandromyia termitophila & MT & Gaúcha do Norte & -53.08 & -13.24 \\
\hline Evandromyia termitophila & MT & General Carneiro & -52.76 & -15.71 \\
\hline Evandromyia termitophila & MT & Guarantã do Norte & -54.90 & -9.78 \\
\hline Evandromyia termitophila & MT & Guiratinga & -53.74 & -16.33 \\
\hline Evandromyia termitophila & MT & Itiquira & -54.14 & -17.21 \\
\hline Evandromyia termitophila & MT & Jaciara & -54.95 & -15.92 \\
\hline Evandromyia termitophila & MT & Jangada & -56.49 & -15.24 \\
\hline Evandromyia termitophila & MT & Juara & -57.52 & -11.55 \\
\hline Evandromyia termitophila & MT & Juína & -58.74 & -11.38 \\
\hline Evandromyia termitophila & MT & Juscimeira & -54.88 & -16.05 \\
\hline Evandromyia termitophila & MT & Mercelândia & -54.60 & -11.11 \\
\hline Evandromyia termitophila & MT & Matupá & -54.90 & -10.05 \\
\hline Evandromyia termitophila & MT & Nobres & -56.33 & -14.73 \\
\hline Evandromyia termitophila & MT & Nortelândia & -56.80 & -14.42 \\
\hline Evandromyia termitophila & MT & Nossa S. do Livramento & -56.37 & -15.80 \\
\hline Evandromyia termitophila & MT & Nova Bandeirantes & -57.86 & -9.95 \\
\hline Evandromyia termitophila & MT & Nova Brasilândia & -54.57 & -14.57 \\
\hline Evandromyia termitophila & MT & Nova Canaã do Norte & -55.95 & -10.55 \\
\hline Evandromyia termitophila & MT & Nova Marilândia & -56.57 & -14.21 \\
\hline Evandromyia termitophila & MT & Nova Maringá & -57.06 & -13.01 \\
\hline Evandromyia termitophila & MT & Nova Mutum & -56.08 & -13.83 \\
\hline Evandromyia termitophila & MT & Nova Santana Helena & -53.35 & -10.82 \\
\hline Evandromyia termitophila & MT & Nova Ubiratã & -55.26 & -13.01 \\
\hline Evandromyia termitophila & MT & Novo Horizonte do Norte & -57.35 & -11.41 \\
\hline Evandromyia termitophila & MT & Novo São Joaquim & -53.02 & -14.90 \\
\hline Evandromyia termitophila & MT & Paranaíta & -56.48 & -9.67 \\
\hline Evandromyia termitophila & MT & Paratinga & -54.05 & -14.43 \\
\hline
\end{tabular}




\begin{tabular}{|c|c|c|c|c|}
\hline Sandfly species & State code & Municipalities & Longitude & Latitude \\
\hline Evandromyia termitophila & MT & Peixoto de Azevedo & -54.98 & -10.22 \\
\hline Evandromyia termitophila & MT & Planalto da Serra & -55.27 & -10.44 \\
\hline Evandromyia termitophila & MT & Poconé & -56.62 & -16.26 \\
\hline Evandromyia termitophila & MT & Pontal do Araguaia & -52.00 & -15.84 \\
\hline Evandromyia termitophila & MT & Ponte Branca & -56.78 & -16.08 \\
\hline Evandromyia termitophila & MT & Porto Alegre do Norte & -51.63 & -10.87 \\
\hline Evandromyia termitophila & MT & Porto dos Gaúchos & -57.41 & -11.52 \\
\hline Evandromyia termitophila & MT & Poxoréo & -54.38 & -15.83 \\
\hline Evandromyia termitophila & MT & Primavera do Leste & -54.30 & -15.56 \\
\hline Evandromyia termitophila & MT & Ribeirãozinho & -52.67 & -16.45 \\
\hline Evandromyia termitophila & MT & Rondonópolis & -54.64 & -16.47 \\
\hline Evandromyia termitophila & MT & Rosário Oeste & -56.43 & -14.84 \\
\hline Evandromyia termitophila & MT & Santa Rita do Trivelato & -55.26 & -13.81 \\
\hline Evandromyia termitophila & MT & São Felix do Araguaia & -50.67 & -11.61 \\
\hline Evandromyia termitophila & MT & São José dos 4 Marcos & -58.17 & -15.17 \\
\hline Evandromyia termitophila & MT & Sapezal & -57.20 & -14.97 \\
\hline Evandromyia termitophila & MT & Serra da Nova Dourado & -51.58 & -11.97 \\
\hline Evandromyia termitophila & MT & Sinop & -55.49 & -11.83 \\
\hline Evandromyia termitophila & MT & Sorriso & -55.71 & -12.55 \\
\hline Evandromyia termitophila & MT & Tamboporã & -56.62 & -10.80 \\
\hline Evandromyia termitophila & MT & Tapurah & -56.50 & -12.53 \\
\hline Evandromyia termitophila & MT & Terra nova do Norte & -55.23 & -10.51 \\
\hline Evandromyia termitophila & MT & Teorixoréu & -52.56 & -16.20 \\
\hline Evandromyia termitophila & MT & União do Sul & -54.36 & -11.52 \\
\hline Evandromyia termitophila & MT & Vila Rica & -51.11 & -10.01 \\
\hline Evandromyia termitophila & GO & Bom Jardim & -52.12 & -16.28 \\
\hline Evandromyia termitophila & GO & Campos Verdes & -49.66 & -14.26 \\
\hline Evandromyia termitophila & $\mathrm{GO}$ & Doverlândia & -52.31 & -16.69 \\
\hline Evandromyia termitophila & $\mathrm{GO}$ & Goiás & -50.14 & -15.93 \\
\hline Evandromyia termitophila & $\mathrm{GO}$ & Mambaí & -46.11 & -14.49 \\
\hline Evandromyia termitophila & GO & Mutunópolis & -49.28 & -13.73 \\
\hline Evandromyia termitophila & GO & Mundo Novo & -40.47 & -11.86 \\
\hline Evandromyia termitophila & GO & Minaçu & -48.22 & -13.53 \\
\hline Evandromyia termitophila & GO & Niquelândia & -48.45 & -14.47 \\
\hline Evandromyia termitophila & $\mathrm{GO}$ & Rio Verde & -50.93 & -17.80 \\
\hline Evandromyia termitophila & $\mathrm{GO}$ & São Domingos & -46.32 & -13.40 \\
\hline Evandromyia termitophila & $\mathrm{GO}$ & Uruaçu & -49.14 & -14.52 \\
\hline Evandromyia termitophila & GO & Itumbiara & -49.22 & -18.42 \\
\hline Evandromyia termitophila & DF & Brasília & -47.42 & -15.78 \\
\hline Evandromyia termitophila & MS & Aquidauana & -55.79 & -20.47 \\
\hline Evandromyia termitophila & MS & Amambai & -55.22 & -23.08 \\
\hline Evandromyia termitophila & MS & Anaurilândia & -52.75 & -22.05 \\
\hline Evandromyia termitophila & MS & Bela Vista & -56.52 & -22.11 \\
\hline Evandromyia termitophila & MS & Bonito & -56.47 & -21.13 \\
\hline Evandromyia termitophila & MS & Brasilândia & -52.04 & -21.26 \\
\hline Evandromyia termitophila & MS & Camapuã & -54.04 & -19.53 \\
\hline Evandromyia termitophila & MS & Campo Grande & -54.65 & -20.44 \\
\hline Evandromyia termitophila & MS & Corguinho & -54.83 & -19.83 \\
\hline Evandromyia termitophila & MS & Paranaíba & -51.19 & -19.68 \\
\hline Evandromyia termitophila & MS & Ponta Porã & -55.73 & -22.54 \\
\hline Evandromyia termitophila & MS & Rochedo & -54.89 & -19.95 \\
\hline Evandromyia termitophila & MS & Selvíria & -51.42 & -20.37 \\
\hline Evandromyia termitophila & MS & Dourados & -54.81 & -22.22 \\
\hline
\end{tabular}




\begin{tabular}{|c|c|c|c|c|}
\hline Sandfly species & State code & Municipalities & Longitude & Latitude \\
\hline Evandromyia termitophila & MS & Itaporã & -54.90 & -22.02 \\
\hline Evandromyia termitophila & MS & Naviraí & -54.22 & -23.13 \\
\hline Evandromyia termitophila & MS & Nova Andradina & -53.34 & -22.23 \\
\hline Evandromyia termitophila & MS & Novo Horizonte do Sul & -53.80 & -22.61 \\
\hline Evandromyia termitophila & MS & Nioaque & -55.83 & -21.14 \\
\hline Evandromyia termitophila & MS & Bonito & -56.47 & -21.13 \\
\hline Evandromyia termitophila & MS & Maracajú & -55.17 & -21.61 \\
\hline Evandromyia termitophila & MS & São Gabriel do Oeste & -54.57 & -19.40 \\
\hline Evandromyia termitophila & MS & Rio Verde de Mato Grosso & -54.84 & -18.92 \\
\hline Evandromyia termitophila & MS & Três Lagoas & -51.68 & -20.75 \\
\hline Evandromyia teratodes & MT & Varzea Grande & -56.13 & -15.65 \\
\hline Evandromyia teratodes & MT & Barra do Garças & -52.26 & -15.89 \\
\hline Evandromyia teratodes & MT & Alto do Paraguai & -56.48 & -14.51 \\
\hline Evandromyia teratodes & MT & Alto Taquarí & -53.16 & -17.49 \\
\hline Evandromyia teratodes & MT & Araguaina & -51.83 & -15.73 \\
\hline Evandromyia teratodes & MT & Barão do Melgaço & -55.97 & -16.19 \\
\hline Evandromyia teratodes & MT & Campinápolis & -52.88 & -14.50 \\
\hline Evandromyia teratodes & MT & Canabrava do Norte & -51.83 & -11.03 \\
\hline Evandromyia teratodes & MT & Chapada dos Guimarães & -55.75 & -15.46 \\
\hline Evandromyia teratodes & MT & Cuiabá & -56.10 & -15.60 \\
\hline Evandromyia teratodes & MT & Diamantino & -56.45 & -14.41 \\
\hline Evandromyia teratodes & MT & Gaúcha do Norte & -53.08 & -13.24 \\
\hline Evandromyia teratodes & MT & Guarantã do Norte & -54.90 & -9.78 \\
\hline Evandromyia teratodes & MT & Jaciara & -54.95 & -15.92 \\
\hline Evandromyia teratodes & MT & Nobres & -56.33 & -14.73 \\
\hline Evandromyia teratodes & MT & Nossa S. do Livramento & -56.37 & -15.80 \\
\hline Evandromyia teratodes & MT & Nova Brasilândia & -54.57 & -14.57 \\
\hline Evandromyia teratodes & MT & Nova Maringá & -57.06 & -13.01 \\
\hline Evandromyia teratodes & MT & Nova Mutum & -56.08 & -13.83 \\
\hline Evandromyia teratodes & MT & Novo São Joaquim & -53.02 & -14.90 \\
\hline Evandromyia teratodes & MT & Peixoto de Azevedo & -54.98 & -10.22 \\
\hline Evandromyia teratodes & MT & Planalto da Serra & -55.27 & -10.44 \\
\hline Evandromyia teratodes & MT & Rondonópolis & -54.64 & -16.47 \\
\hline Evandromyia teratodes & MT & Poxoréo & -54.38 & -15.83 \\
\hline Evandromyia teratodes & MT & São Felix do Araguaia & -50.67 & -11.61 \\
\hline Evandromyia teratodes & MT & São José dos 4 Marcos & -50.67 & -11.61 \\
\hline Evandromyia teratodes & MT & Serra da Nova Dourado & -51.58 & -11.97 \\
\hline Evandromyia teratodes & MT & Tangará da Serra & -57.50 & -14.62 \\
\hline Evandromyia teratodes & MT & Teorixoréu & -52.56 & -16.20 \\
\hline Evandromyia teratodes & GO & Itumbiara & -49.22 & -18.42 \\
\hline Evandromyia teratodes & $\mathrm{GO}$ & Itapaci & -49.57 & -14.95 \\
\hline Evandromyia teratodes & DF & Brasília & -47.42 & -15.78 \\
\hline Evandromyia teratodes & MS & Campo Grande & -54.65 & -20.44 \\
\hline Evandromyia teratodes & MS & Corguinho & -54.83 & -19.83 \\
\hline Evandromyia teratodes & MS & Rio Negro & -54.99 & -19.45 \\
\hline Evandromyia teratodes & MS & Nova Andradina & -53.34 & -22.23 \\
\hline Evandromyia teratodes & MS & Rio Verde de Mato Grosso & -54.84 & -18.92 \\
\hline Evandromyia teratodes & MS & Bonito & -56.47 & -21.13 \\
\hline Evandromyia walkeri & MT & Barra dos Bugres & -57.18 & -15.08 \\
\hline Evandromyia walkeri & MT & Rosário d' Oeste & -56.43 & -14.84 \\
\hline Evandromyia walkeri & MT & Varzea Grande & -56.13 & -15.65 \\
\hline Evandromyia walkeri & MT & Nova Mutum & -56.08 & -13.83 \\
\hline Evandromyia walkeri & MT & Cuiabá & -56.10 & -15.60 \\
\hline
\end{tabular}




\begin{tabular}{|c|c|c|c|c|}
\hline Sandfly species & State code & Municipalities & Longitude & Latitude \\
\hline Evandromyia walkeri & MT & Barra do Garças & -52.26 & -15.89 \\
\hline Evandromyia walkeri & MT & Acorizal & -56.37 & -15.20 \\
\hline Evandromyia walkeri & MT & Alta Floresta & -56.09 & -9.88 \\
\hline Evandromyia walkeri & MT & Alto do Paraguai & -56.48 & -14.51 \\
\hline Evandromyia walkeri & MT & Alto Taquarí & -53.16 & -17.49 \\
\hline Evandromyia walkeri & MT & Araguaina & -51.83 & -15.73 \\
\hline Evandromyia walkeri & MT & Arenápolis & -56.82 & -14.43 \\
\hline Evandromyia walkeri & MT & Aripuanã & -60.63 & -9.17 \\
\hline Evandromyia walkeri & MT & Barão do Melgaço & -55.97 & -16.19 \\
\hline Evandromyia walkeri & MT & Campo Novo do Parecis & -57.88 & -13.67 \\
\hline Evandromyia walkeri & MT & Campos de Julio & -59.08 & -13.53 \\
\hline Evandromyia walkeri & MT & Canabrava do Norte & -51.83 & -11.03 \\
\hline Evandromyia walkeri & MT & Chapada dos Guimarães & -55.75 & -15.46 \\
\hline Evandromyia walkeri & MT & Claúdia & -54.88 & -11.49 \\
\hline Evandromyia walkeri & MT & Colíder & -55.45 & -10.81 \\
\hline Evandromyia walkeri & MT & Colniza & -59.03 & -9.40 \\
\hline Evandromyia walkeri & MT & Comodoro & -59.79 & -13.66 \\
\hline Evandromyia walkeri & MT & Confresa & -51.56 & -10.64 \\
\hline Evandromyia walkeri & MT & Diamantino & -56.45 & -14.41 \\
\hline Evandromyia walkeri & MT & Feliz Natal & -54.92 & -12.39 \\
\hline Evandromyia walkeri & MT & Gaúcha do Norte & -53.08 & -13.24 \\
\hline Evandromyia walkeri & MT & Gerneral Carneiro & -52.76 & -15.71 \\
\hline Evandromyia walkeri & MT & Guarantã do Norte & -54.90 & -9.78 \\
\hline Evandromyia walkeri & MT & Itiquira & -54.14 & -17.21 \\
\hline Evandromyia walkeri & MT & Jaciara & -54.95 & -15.92 \\
\hline Evandromyia walkeri & MT & Juína & -58.74 & -11.38 \\
\hline Evandromyia walkeri & MT & Lucas do Rio Verde & -55.91 & -13.05 \\
\hline Evandromyia walkeri & MT & Luciara & -50.57 & -10.75 \\
\hline Evandromyia walkeri & MT & Nobres & -56.33 & -14.73 \\
\hline Evandromyia walkeri & MT & Nova Maringá & -57.06 & -13.01 \\
\hline Evandromyia walkeri & MT & Nova Ubiratã & -55.26 & -13.01 \\
\hline Evandromyia walkeri & MT & Novo Horizonte do Norte & -57.35 & -11.41 \\
\hline Evandromyia walkeri & MT & Paranaíta & -56.48 & -9.67 \\
\hline Evandromyia walkeri & MT & Peixoto de Azevedo & -54.98 & -10.22 \\
\hline Evandromyia walkeri & MT & Planalto da Serra & -55.27 & -10.44 \\
\hline Evandromyia walkeri & MT & Pontal do Araguaia & -52.01 & -15.85 \\
\hline Evandromyia walkeri & MT & Pontes de Lacerda & -59.37 & -15.20 \\
\hline Evandromyia walkeri & MT & Porto Alegre do Norte & -51.63 & -10.87 \\
\hline Evandromyia walkeri & MT & Porto dos Gaúchos & -57.41 & -11.52 \\
\hline Evandromyia walkeri & MT & Porto Estrela & -57.22 & -15.32 \\
\hline Evandromyia walkeri & MT & Rondonópolis & -54.64 & -16.47 \\
\hline Evandromyia walkeri & MT & Santa Rita do Trivelato & -55.26 & -13.81 \\
\hline Evandromyia walkeri & MT & São Felix do Araguaia & -50.67 & -11.61 \\
\hline Evandromyia walkeri & MT & São José dos 4 Marcos & -58.17 & -15.17 \\
\hline Evandromyia walkeri & MT & Sinop & -55.49 & -11.83 \\
\hline Evandromyia walkeri & MT & Sorriso & -55.71 & -12.55 \\
\hline Evandromyia walkeri & MT & Tapurah & -56.50 & -12.53 \\
\hline Evandromyia walkeri & MT & Teorixoréu & -52.56 & -16.20 \\
\hline Evandromyia walkeri & MT & União do Sul & -54.36 & -11.52 \\
\hline Evandromyia walkeri & MT & Vera & -55.19 & -12.18 \\
\hline Evandromyia walkeri & MT & Vila Bela de S. Trindade & -59.95 & -15.00 \\
\hline Evandromyia walkeri & MT & Ipiranga do Norte & -56.05 & -15.35 \\
\hline Evandromyia walkeri & GO & Itumbiara & -49.22 & -18.42 \\
\hline
\end{tabular}




\begin{tabular}{|c|c|c|c|c|}
\hline Sandfly species & State code & Municipalities & Longitude & Latitude \\
\hline Evandromyia walkeri & MS & Terenos & -54.86 & -20.44 \\
\hline Evandromyia walkeri & MS & Nioaque & -55.83 & -21.14 \\
\hline Evandromyia walkeri & MS & Corumbá & -57.65 & -19.01 \\
\hline Evandromyia sallesi & MT & Rosário d' Oeste & -56.42 & -14.83 \\
\hline Evandromyia sallesi & MT & Varzea Grande & -56.13 & -15.65 \\
\hline Evandromyia sallesi & MT & Nova Mutum & -56.08 & -13.83 \\
\hline Evandromyia sallesi & MT & Cuiabá & -56.10 & -15.60 \\
\hline Evandromyia sallesi & MT & Barra do Garças & -52.26 & -15.89 \\
\hline Evandromyia sallesi & MT & Acorizal & -56.37 & -15.20 \\
\hline Evandromyia sallesi & MT & Alta Floresta & -56.09 & -9.88 \\
\hline Evandromyia sallesi & MT & Alto da Boa Vista & -51.38 & -11.66 \\
\hline Evandromyia sallesi & MT & Alto do Paraguai & -56.48 & -14.51 \\
\hline Evandromyia sallesi & MT & Alto Taquarí & -53.16 & -17.49 \\
\hline Evandromyia sallesi & MT & Araguaina & -51.83 & -15.73 \\
\hline Evandromyia sallesi & MT & Barão do Melgaço & -55.97 & -16.19 \\
\hline Evandromyia sallesi & MT & Barra do Garças & -52.26 & -15.89 \\
\hline Evandromyia sallesi & MT & Campinápolis & -52.88 & -14.50 \\
\hline Evandromyia sallesi & MT & Canabrava do Norte & -51.83 & -11.03 \\
\hline Evandromyia sallesi & MT & Canarana & -52.27 & -13.55 \\
\hline Evandromyia sallesi & MT & Chapada dos Guimarães & -55.75 & -15.46 \\
\hline Evandromyia sallesi & MT & Confresa & -51.56 & -10.64 \\
\hline Evandromyia sallesi & MT & Diamantino & -56.45 & -14.41 \\
\hline Evandromyia sallesi & MT & Gaúcha do Norte & -53.08 & -13.24 \\
\hline Evandromyia sallesi & MT & Gerneral Carneiro & -52.76 & -15.71 \\
\hline Evandromyia sallesi & MT & Itiquira & -54.14 & -17.21 \\
\hline Evandromyia sallesi & MT & Jaciara & -54.95 & -15.92 \\
\hline Evandromyia sallesi & MT & Jangada & -56.49 & -15.24 \\
\hline Evandromyia sallesi & MT & Juscimeira & -54.88 & -16.05 \\
\hline Evandromyia sallesi & MT & Mercelândia & -54.60 & -11.11 \\
\hline Evandromyia sallesi & MT & Mirassol d' Oeste & -58.05 & -15.40 \\
\hline Evandromyia sallesi & MT & Nobres & -56.33 & -14.73 \\
\hline Evandromyia sallesi & MT & Nossa S. do Livramento & -56.37 & -15.80 \\
\hline Evandromyia sallesi & MT & Nova Brasilândia & -54.57 & -14.57 \\
\hline Evandromyia sallesi & MT & Nova Marilândia & -56.57 & -14.21 \\
\hline Evandromyia sallesi & MT & Nova Maringá & -57.06 & -13.01 \\
\hline Evandromyia sallesi & MT & Novo São Joaquim & -53.02 & -14.90 \\
\hline Evandromyia sallesi & MT & Paratinga & -54.05 & -14.43 \\
\hline Evandromyia sallesi & MT & Pedra Preta & -54.47 & -16.62 \\
\hline Evandromyia sallesi & MT & Peixoto de Azevedo & -54.98 & -10.22 \\
\hline Evandromyia sallesi & MT & Planalto da Serra & -55.27 & -10.44 \\
\hline Evandromyia sallesi & MT & Poconé & -56.62 & -16.26 \\
\hline Evandromyia sallesi & MT & Pontal do Araguaia & -52.01 & -15.85 \\
\hline Evandromyia sallesi & MT & Ponte Branca & -56.78 & -16.08 \\
\hline Evandromyia sallesi & MT & Porto Alegre do Norte & -51.63 & -10.87 \\
\hline Evandromyia sallesi & MT & Poxoréo & -54.38 & -15.83 \\
\hline Evandromyia sallesi & MT & Primavera do Leste & -54.30 & -15.56 \\
\hline Evandromyia sallesi & MT & Ribeirão Cascalheira & -51.82 & -12.92 \\
\hline Evandromyia sallesi & MT & Rondonópolis & -54.64 & -16.47 \\
\hline Evandromyia sallesi & MT & Santo Antônio do Leste & -53.62 & -14.83 \\
\hline Evandromyia sallesi & MT & Santo Antônio do Leverger & -56.08 & -15.87 \\
\hline Evandromyia sallesi & MT & São Felix do Araguaia & -50.67 & -11.61 \\
\hline Evandromyia sallesi & MT & São José dos 4 Marcos & -58.17 & -15.17 \\
\hline Evandromyia sallesi & MT & Sinop & -55.49 & -11.83 \\
\hline
\end{tabular}




\begin{tabular}{|c|c|c|c|c|}
\hline Sandfly species & State code & Municipalities & Longitude & Latitude \\
\hline Evandromyia sallesi & MT & Sorriso & -55.71 & -12.55 \\
\hline Evandromyia sallesi & MT & Tangará da Serra & -57.50 & -14.62 \\
\hline Evandromyia sallesi & MT & Teorixoréu & -52.56 & -16.20 \\
\hline Evandromyia sallesi & MT & União do Sul & -54.36 & -11.52 \\
\hline Evandromyia sallesi & MT & Vera & -55.19 & -12.18 \\
\hline Evandromyia sallesi & MT & Vila Bela de S. Trindade & -59.95 & -15.00 \\
\hline Evandromyia sallesi & MT & Vila Rica & -51.11 & -10.01 \\
\hline Evandromyia sallesi & GO & Campos do Rio Verde & -49.70 & -15.35 \\
\hline Evandromyia sallesi & $\mathrm{GO}$ & Campos Verdes & -49.66 & -14.26 \\
\hline Evandromyia sallesi & GO & Campestre de Goiás & -49.69 & -16.73 \\
\hline Evandromyia sallesi & GO & Cumari & -48.15 & -18.26 \\
\hline Evandromyia sallesi & GO & Crixás & -49.97 & -14.45 \\
\hline Evandromyia sallesi & GO & Doverlândia & -52.31 & -16.69 \\
\hline Evandromyia sallesi & GO & Goiatuba & -49.37 & -18.02 \\
\hline Evandromyia sallesi & GO & Guapó & -49.53 & -16.83 \\
\hline Evandromyia sallesi & GO & Mambaí & -46.11 & -14.49 \\
\hline Evandromyia sallesi & GO & Montividiu do Norte & -48.61 & -13.11 \\
\hline Evandromyia sallesi & GO & Mundo Novo & -40.47 & -11.86 \\
\hline Evandromyia sallesi & GO & Minaçu & -48.22 & -13.53 \\
\hline Evandromyia sallesi & GO & Niquelândia & -48.46 & -14.47 \\
\hline Evandromyia sallesi & GO & Nova Crixás & -50.33 & -14.10 \\
\hline Evandromyia sallesi & GO & Porangatu & -49.15 & -13.44 \\
\hline Evandromyia sallesi & GO & Rio Verde & -50.93 & -17.80 \\
\hline Evandromyia sallesi & GO & São Domingos & -46.32 & -13.40 \\
\hline Evandromyia sallesi & GO & Silvânia & -48.61 & -16.66 \\
\hline Evandromyia sallesi & GO & Uriapuru & -49.92 & -14.29 \\
\hline Evandromyia sallesi & GO & Itumbiara & -49.22 & -18.42 \\
\hline Evandromyia sallesi & GO & Sítio d' Abadia & -46.25 & -14.80 \\
\hline Evandromyia sallesi & GO & Amaro Leite & -49.15 & -13.97 \\
\hline Evandromyia sallesi & GO & Ceres & -49.59 & -15.30 \\
\hline Evandromyia sallesi & GO & Rianópolis & -49.47 & -15.48 \\
\hline Evandromyia sallesi & GO & Anápolis & -48.95 & -16.32 \\
\hline Evandromyia sallesi & GO & Jaraguá & -49.33 & -15.75 \\
\hline Evandromyia sallesi & $\mathrm{DF}$ & Brasília & -47.42 & -15.78 \\
\hline Evandromyia sallesi & MS & Água Clara & -52.87 & -20.44 \\
\hline Evandromyia sallesi & MS & Alcinópolis & -53.70 & -18.31 \\
\hline Evandromyia sallesi & MS & Anastácio & -55.81 & -20.48 \\
\hline Evandromyia sallesi & MS & Antonio João & -55.95 & -22.19 \\
\hline Evandromyia sallesi & MS & Aquidauana & -55.79 & -20.47 \\
\hline Evandromyia sallesi & MS & Bodoquena & -56.72 & -20.54 \\
\hline Evandromyia sallesi & MS & Camapuã & -54.04 & -19.53 \\
\hline Evandromyia sallesi & MS & Corumbá & -57.65 & -19.01 \\
\hline Evandromyia sallesi & MS & Ponta Porã & -55.73 & -22.54 \\
\hline Evandromyia sallesi & MS & São Gabriel do Oeste & -54.57 & -19.40 \\
\hline Evandromyia sallesi & MS & Selvíria & -51.42 & -20.37 \\
\hline Evandromyia sallesi & MS & Terenos & -54.86 & -20.44 \\
\hline Evandromyia sallesi & MS & Costa Rica & -53.13 & -18.54 \\
\hline Evandromyia sallesi & MS & Guia Lopes da Laguna & -56.11 & -21.46 \\
\hline Evandromyia sallesi & MS & Jaraguarí & -54.40 & -20.14 \\
\hline Evandromyia sallesi & MS & Nova Andradina & -53.34 & -22.23 \\
\hline Evandromyia sallesi & MS & Novo Horizonte do Sul & -53.80 & -22.61 \\
\hline Evandromyia sallesi & MS & Nioaque & -55.83 & -21.13 \\
\hline Evandromyia sallesi & MS & Bonito & -56.47 & -21.13 \\
\hline
\end{tabular}




\begin{tabular}{|c|c|c|c|c|}
\hline Sandfly species & State code & Municipalities & Longitude & Latitude \\
\hline Evandromyia sallesi & MS & Maracajú & -55.17 & -21.61 \\
\hline Evandromyia sallesi & MS & Miranda & -56.38 & -20.24 \\
\hline Evandromyia bacula & $\mathrm{GO}$ & Itumbiara & -49.22 & -18.42 \\
\hline Evandromyia bacula & MT & Caúdia & -54.88 & -11.49 \\
\hline Evandromyia bacula & MT & Chapada dos Guimarães & -55.75 & -15.46 \\
\hline Evandromyia bacula & MT & Carolina & -55.83 & -9.96 \\
\hline Evandromyia bacula & MT & Colíder & -55.45 & -10.81 \\
\hline Evandromyia bacula & MT & Cotriguaçu & -58.41 & -9.85 \\
\hline Evandromyia bacula & MT & Gaúcha do Norte & -53.08 & -13.24 \\
\hline Evandromyia bacula & MT & Guarantã do Norte & -54.90 & -9.78 \\
\hline Evandromyia bacula & MT & Juína & -58.74 & -11.38 \\
\hline Evandromyia bacula & MT & Mercelândia & -54.60 & -11.11 \\
\hline Evandromyia bacula & MT & Nova Bandeirantes & -57.86 & -9.95 \\
\hline Evandromyia bacula & MT & Nova Brasilândia & -54.97 & -14.96 \\
\hline Evandromyia bacula & MT & Nova Canaã do Norte & -55.95 & -10.55 \\
\hline Evandromyia bacula & MT & Nova Mutum & -56.08 & -13.83 \\
\hline Evandromyia bacula & MT & Paranaíta & -57.41 & -11.52 \\
\hline Evandromyia bacula & MT & Sinop & -55.49 & -11.83 \\
\hline Evandromyia bacula & MT & Tapurah & -56.50 & -12.53 \\
\hline Evandromyia bacula & MT & União do Sul & -54.36 & -11.52 \\
\hline Evandromyia bacula & MT & Várzea Grande & -56.13 & -15.65 \\
\hline Evandromyia bacula & MS & Santa Rita do Pardo & -52.83 & -21.30 \\
\hline Evandromyia bacula & DF & Brasília & -47.42 & -15.78 \\
\hline Lutzomyia cruzi & MS & Alcinópolis & -53.70 & -18.31 \\
\hline Lutzomyia cruzi & MS & Bonito & -56.47 & -21.13 \\
\hline Lutzomyia cruzi & MS & Camapuã & -54.04 & -19.53 \\
\hline Lutzomyia cruzi & MS & Cassilândia & -51.73 & -19.11 \\
\hline Lutzomyia cruzi & MS & Corumbá & -57.65 & -19.01 \\
\hline Lutzomyia cruzi & MS & Campo Grande & -54.65 & -20.44 \\
\hline Lutzomyia cruzi & MS & Paraíso das Águas & -52.97 & -19.05 \\
\hline Lutzomyia cruzi & MS & Ladário & -57.60 & -19.00 \\
\hline Lutzomyia cruzi & MS & São Gabriel d Oeste & -54.57 & -19.40 \\
\hline Lutzomyia cruzi & MT & Alto Araguaia & -53.22 & -17.31 \\
\hline Lutzomyia cruzi & MT & Araguaiana & -51.83 & -15.73 \\
\hline Lutzomyia cruzi & MT & Barra dos Bugres & -57.18 & -15.08 \\
\hline Lutzomyia cruzi & MT & Barra do Garças & -52.26 & -15.89 \\
\hline Lutzomyia cruzi & MT & Barão de Melgaço & -55.97 & -16.19 \\
\hline Lutzomyia cruzi & MT & Canarana & -52.27 & -13.55 \\
\hline Lutzomyia cruzi & MT & Colider & -55.45 & -10.81 \\
\hline Lutzomyia cruzi & MT & Cáceres & -57.68 & -16.07 \\
\hline Lutzomyia cruzi & MT & Chapada dos Guimarães & -55.75 & -15.46 \\
\hline Lutzomyia cruzi & MT & Cuiabá & -56.10 & -15.60 \\
\hline Lutzomyia cruzi & MT & Dom Aquino & -54.92 & -15.81 \\
\hline Lutzomyia cruzi & MT & Sapezal & -58.76 & -12.98 \\
\hline Lutzomyia cruzi & MT & Feliz Natal & -54.92 & -12.39 \\
\hline Lutzomyia cruzi & MT & General Carneiro & -52.76 & -15.71 \\
\hline Lutzomyia cruzi & MT & Guiratinga & -53.76 & -16.35 \\
\hline Lutzomyia cruzi & MT & Jaciara & -54.97 & -15.97 \\
\hline Lutzomyia cruzi & MT & Juína & -58.74 & -11.38 \\
\hline Lutzomyia cruzi & MT & Itiquira & -54.15 & -17.21 \\
\hline Lutzomyia cruzi & MT & Juscimeira & -54.88 & -16.05 \\
\hline Lutzomyia cruzi & MT & Mirasol D' Oeste & -58.05 & -15.40 \\
\hline Lutzomyia cruzi & MT & Nobres & -56.33 & -14.72 \\
\hline
\end{tabular}




\begin{tabular}{|c|c|c|c|c|}
\hline Sandfly species & State code & Municipalities & Longitude & Latitude \\
\hline Lutzomyia cruzi & MT & Nova Brasilândia & -54.97 & -14.96 \\
\hline Lutzomyia cruzi & MT & Nova Canaã do Norte & -55.95 & -10.56 \\
\hline Lutzomyia cruzi & MT & Nova Ubiratã & -55.25 & -12.99 \\
\hline Lutzomyia cruzi & MT & Novo São Joaquim & -53.02 & -14.90 \\
\hline Lutzomyia cruzi & MT & Pedra Preta & -54.47 & -16.62 \\
\hline Lutzomyia cruzi & MT & Paranatinga & -54.05 & -14.43 \\
\hline Lutzomyia cruzi & MT & Poconé & -56.62 & -16.26 \\
\hline Lutzomyia cruzi & MT & Pontal do Araguaia & -52.01 & -15.85 \\
\hline Lutzomyia cruzi & MT & Poxoréo & -54.39 & -15.84 \\
\hline Lutzomyia cruzi & MT & Primavera do Leste & -54.30 & -15.56 \\
\hline Lutzomyia cruzi & MT & Reserva do Cabaçal & -58.43 & -15.08 \\
\hline Lutzomyia cruzi & MT & Rondolândia & -55.42 & -14.50 \\
\hline Lutzomyia cruzi & MT & Rondonópolis & -54.64 & -16.47 \\
\hline Lutzomyia cruzi & MT & Rosário Oeste & -56.43 & -14.84 \\
\hline Lutzomyia cruzi & MT & Santa Rita do Trivelato & -55.26 & -13.81 \\
\hline Lutzomyia cruzi & MT & Santo Antônio do Leverger & -56.08 & -15.87 \\
\hline Lutzomyia cruzi & MT & São José dos 4 Marcos & -58.18 & -15.62 \\
\hline Lutzomyia cruzi & MT & Sorriso & -55.71 & -12.55 \\
\hline Lutzomyia cruzi & MT & Sinop & -55.50 & -11.86 \\
\hline Lutzomyia cruzi & MT & Torixoreu & -52.56 & -16.20 \\
\hline Lutzomyia cruzi & MT & União do Sul & -54.35 & -11.53 \\
\hline Lutzomyia cruzi & $\mathrm{GO}$ & Paraúna & -50.43 & -17.03 \\
\hline Lutzomyia longipalpis & MT & Água Boa & -52.16 & -14.05 \\
\hline Lutzomyia longipalpis & MT & Alta Floresta & -56.09 & -9.88 \\
\hline Lutzomyia longipalpis & MT & Peixoto de Azevedo & -54.98 & -10.22 \\
\hline Lutzomyia longipalpis & MT & Colider & -55.45 & -10.81 \\
\hline Lutzomyia longipalpis & MT & Comodoro & -59.79 & -13.66 \\
\hline Lutzomyia longipalpis & MT & Alto Araguaia & -53.22 & -17.31 \\
\hline Lutzomyia longipalpis & MT & Mirassol D`Oeste & -58.05 & -15.40 \\
\hline Lutzomyia longipalpis & MT & Diamantino & -56.45 & -14.41 \\
\hline Lutzomyia longipalpis & MT & Alto Paraguai & -56.48 & -14.51 \\
\hline Lutzomyia longipalpis & MT & Chapada dos Guimarães & -55.75 & -15.46 \\
\hline Lutzomyia longipalpis & MT & Feliz Natal & -54.92 & -12.39 \\
\hline Lutzomyia longipalpis & MT & Jangada & -56.49 & -15.24 \\
\hline Lutzomyia longipalpis & MT & Várzea Grande & -56.13 & -15.65 \\
\hline Lutzomyia longipalpis & MT & Nossa S. do Livramento & -56.35 & -15.78 \\
\hline Lutzomyia longipalpis & MT & Nobres & -56.33 & -14.72 \\
\hline Lutzomyia longipalpis & MT & Juína & -58.74 & -11.38 \\
\hline Lutzomyia longipalpis & MT & Santo Antônio do Leverger & -56.08 & -15.87 \\
\hline Lutzomyia longipalpis & MT & Rondonópolis & -54.64 & -16.47 \\
\hline Lutzomyia longipalpis & MT & Sorriso & -55.71 & -12.55 \\
\hline Lutzomyia longipalpis & MT & Itanhangá & -56.64 & -12.23 \\
\hline Lutzomyia longipalpis & MT & União do Sul & -54.36 & -11.52 \\
\hline Lutzomyia longipalpis & MT & Terra Nova do Norte & -55.23 & -10.51 \\
\hline Lutzomyia longipalpis & MT & Tapurah & -56.50 & -12.53 \\
\hline Lutzomyia longipalpis & MT & Itiquira & -54.12 & -17.20 \\
\hline Lutzomyia longipalpis & MT & Planalto da Serra & -55.27 & -10.44 \\
\hline Lutzomyia longipalpis & MT & Nova Mutum & -56.08 & -13.83 \\
\hline Lutzomyia longipalpis & MT & Nova Ubiratã & -55.26 & -13.01 \\
\hline Lutzomyia longipalpis & MT & Nova Xavantina & -52.34 & -14.66 \\
\hline Lutzomyia longipalpis & MT & Paranaíta & -56.48 & -9.67 \\
\hline Lutzomyia longipalpis & MT & Porto Alegre do Norte & -51.63 & -10.87 \\
\hline Lutzomyia longipalpis & MT & Sinop & -55.49 & -11.83 \\
\hline
\end{tabular}




\begin{tabular}{|c|c|c|c|c|}
\hline Sandfly species & State code & Municipalities & Longitude & Latitude \\
\hline Lutzomyia longipalpis & MT & Santa Rita do Trivelato & -55.26 & -13.81 \\
\hline Lutzomyia longipalpis & MT & Gaúcha do Norte & -53.08 & -13.24 \\
\hline Lutzomyia longipalpis & MT & Marcelândia & -54.60 & -11.11 \\
\hline Lutzomyia longipalpis & MT & General Carneiro & -52.76 & -15.71 \\
\hline Lutzomyia longipalpis & MT & Paranatinga & -54.05 & -14.43 \\
\hline Lutzomyia longipalpis & MT & Nova Brasilândia & -54.97 & -14.96 \\
\hline Lutzomyia longipalpis & MT & Rosário D’ Oeste & -56.43 & -14.84 \\
\hline Lutzomyia longipalpis & MT & Pontal do Araguaia & -52.01 & -15.85 \\
\hline Lutzomyia longipalpis & MT & Primavera do Leste & -54.30 & -15.56 \\
\hline Lutzomyia longipalpis & MT & Poconé & -56.62 & -16.26 \\
\hline Lutzomyia longipalpis & MT & Lucas do Rio Verde & -55.91 & -13.05 \\
\hline Lutzomyia longipalpis & MT & Barra dos Bugres & -57.18 & -15.08 \\
\hline Lutzomyia longipalpis & MT & Barra do Garças & -52.26 & -15.89 \\
\hline Lutzomyia longipalpis & MT & Barão de Melgaço & -55.97 & -16.19 \\
\hline Lutzomyia longipalpis & MT & Poxoréu & -54.38 & -15.83 \\
\hline Lutzomyia longipalpis & MT & Araguaiana & -51.83 & -15.73 \\
\hline Lutzomyia longipalpis & MT & Arenápolis & -56.82 & -14.43 \\
\hline Lutzomyia longipalpis & MT & Cuiabá & -56.10 & -15.60 \\
\hline Lutzomyia longipalpis & MT & Barra do Garças & -52.26 & -15.89 \\
\hline Lutzomyia longipalpis & MT & Cáceres & -57.68 & -16.07 \\
\hline Lutzomyia longipalpis & MT & Campinápolis & -52.88 & -14.50 \\
\hline Lutzomyia longipalpis & MT & Canabrava do Norte & -51.83 & -11.03 \\
\hline Lutzomyia longipalpis & MT & Canarana & -52.27 & -13.55 \\
\hline Lutzomyia longipalpis & MT & Cláudia & -54.88 & -11.49 \\
\hline Lutzomyia longipalpis & MT & Àgua Clara & -52.87 & -20.44 \\
\hline Lutzomyia longipalpis & MS & Alcinópolis & -53.70 & -18.31 \\
\hline Lutzomyia longipalpis & MS & Anastácio & -55.81 & -20.48 \\
\hline Lutzomyia longipalpis & MS & Antonio João & -55.95 & -22.19 \\
\hline Lutzomyia longipalpis & MS & Aparecida do Taboado & -51.09 & -20.09 \\
\hline Lutzomyia longipalpis & MS & Aquidauana & -55.79 & -20.47 \\
\hline Lutzomyia longipalpis & MS & Bataguassu & -52.42 & -21.71 \\
\hline Lutzomyia longipalpis & MS & Bela Vista & -56.52 & -22.11 \\
\hline Lutzomyia longipalpis & MS & Bodoquena & -56.72 & -20.54 \\
\hline Lutzomyia longipalpis & MS & Brasilândia & -52.04 & -21.26 \\
\hline Lutzomyia longipalpis & MS & Campo Grande & -54.65 & -20.44 \\
\hline Lutzomyia longipalpis & MS & Camapuã & -54.04 & -19.53 \\
\hline Lutzomyia longipalpis & MS & Corguinho & -54.83 & -19.83 \\
\hline Lutzomyia longipalpis & MS & Caracol & -57.02 & -22.01 \\
\hline Lutzomyia longipalpis & MS & Coxim & -54.76 & -18.51 \\
\hline Lutzomyia longipalpis & MS & Paranaíba & -51.19 & -19.68 \\
\hline Lutzomyia longipalpis & MS & Ponta Porã & -55.73 & -22.54 \\
\hline Lutzomyia longipalpis & MS & Porto Murtinho & -57.88 & -21.70 \\
\hline Lutzomyia longipalpis & MS & Ribas do Rio Pardo & -53.76 & -20.44 \\
\hline Lutzomyia longipalpis & MS & Rio Negro & -54.99 & -19.45 \\
\hline Lutzomyia longipalpis & MS & Rochedo & -54.89 & -19.95 \\
\hline Lutzomyia longipalpis & MS & Selvíria & -51.42 & -20.37 \\
\hline Lutzomyia longipalpis & MS & Sidrolândia & -54.96 & -20.93 \\
\hline Lutzomyia longipalpis & MS & Terenos & -54.86 & -20.44 \\
\hline Lutzomyia longipalpis & MS & Três Lagoas & -51.68 & -20.75 \\
\hline Lutzomyia longipalpis & MS & Costa Rica & -53.13 & -18.54 \\
\hline Lutzomyia longipalpis & MS & Dourados & -54.81 & -22.22 \\
\hline Lutzomyia longipalpis & MS & Guia Lopes da Laguna & -56.11 & -21.46 \\
\hline Lutzomyia longipalpis & MS & Inocência & -51.93 & -19.73 \\
\hline
\end{tabular}




\begin{tabular}{|c|c|c|c|c|}
\hline Sandfly species & State code & Municipalities & Longitude & Latitude \\
\hline Lutzomyia longipalpis & MS & Jaraguarí & -54.40 & -20.14 \\
\hline Lutzomyia longipalpis & MS & Jardim & -56.14 & -21.48 \\
\hline Lutzomyia longipalpis & MS & Nova Alvorada do Sul & -54.38 & -21.47 \\
\hline Lutzomyia longipalpis & MS & Nioaque & -55.83 & -21.14 \\
\hline Lutzomyia longipalpis & MS & Santa Rita do Pardo & -52.83 & -21.30 \\
\hline Lutzomyia longipalpis & MS & Bonito & -56.47 & -21.13 \\
\hline Lutzomyia longipalpis & MS & Dois Irmãos do Buriti & -55.30 & -20.68 \\
\hline Lutzomyia longipalpis & MS & Maracajú & -55.17 & -21.61 \\
\hline Lutzomyia longipalpis & MS & Miranda & -56.38 & -20.24 \\
\hline Lutzomyia longipalpis & MS & Pedro Gomes & -54.55 & -18.10 \\
\hline Lutzomyia longipalpis & MS & Paraíso das Águas & -52.97 & -19.05 \\
\hline Lutzomyia longipalpis & MS & Paranhos & -55.43 & -23.89 \\
\hline Lutzomyia longipalpis & MS & Rio Verde de Mato Grosso & -54.84 & -18.92 \\
\hline Lutzomyia longipalpis & GO & Amaro Leite & -49.15 & -13.97 \\
\hline Lutzomyia longipalpis & GO & Araguapaz & -50.63 & -15.09 \\
\hline Lutzomyia longipalpis & GO & Campos Verdes & -49.66 & -14.26 \\
\hline Lutzomyia longipalpis & GO & Crixás & -49.97 & -14.45 \\
\hline Lutzomyia longipalpis & GO & Padre Bernado & -48.50 & -13.35 \\
\hline Lutzomyia longipalpis & GO & Cavalcante & -47.50 & -13.80 \\
\hline Lutzomyia longipalpis & GO & Campestre & -49.69 & -16.73 \\
\hline Lutzomyia longipalpis & GO & Colinas do Sul & -48.08 & -14.15 \\
\hline Lutzomyia longipalpis & GO & Cumari & -48.15 & -18.26 \\
\hline Lutzomyia longipalpis & GO & Catalão & -47.95 & -18.17 \\
\hline Lutzomyia longipalpis & GO & Flores de Goiás & -47.05 & -14.45 \\
\hline Lutzomyia longipalpis & GO & Goiás & -50.14 & -15.93 \\
\hline Lutzomyia longipalpis & GO & Guapó & -49.53 & -16.83 \\
\hline Lutzomyia longipalpis & GO & Mambaí & -46.11 & -14.49 \\
\hline Lutzomyia longipalpis & GO & Montividiu do Norte & -48.61 & -13.11 \\
\hline Lutzomyia longipalpis & GO & Mutunópolis & -49.28 & -13.73 \\
\hline Lutzomyia longipalpis & GO & Mundo Novo & -40.47 & -11.86 \\
\hline Lutzomyia longipalpis & GO & Minaçu & -48.22 & -13.53 \\
\hline Lutzomyia longipalpis & GO & Morro Agudo de Goiás & -50.06 & -15.32 \\
\hline Lutzomyia longipalpis & GO & Niquelândia & -48.46 & -14.47 \\
\hline Lutzomyia longipalpis & GO & Porangatu & -49.15 & -13.44 \\
\hline Lutzomyia longipalpis & GO & Posse & -46.37 & -14.09 \\
\hline Lutzomyia longipalpis & GO & Rio Verde & -50.93 & -17.80 \\
\hline Lutzomyia longipalpis & GO & São Miguel do Araguaia & -50.22 & -13.32 \\
\hline Lutzomyia longipalpis & GO & São Domingos & -46.32 & -13.40 \\
\hline Lutzomyia longipalpis & GO & Silvânia & -48.61 & -16.66 \\
\hline Lutzomyia longipalpis & GO & Uriapuru & -49.92 & -14.29 \\
\hline Lutzomyia longipalpis & GO & Paraúna & -50.44 & -16.94 \\
\hline Lutzomyia longipalpis & GO & Sítio d' Abadia & -46.25 & -14.80 \\
\hline Lutzomyia longipalpis & GO & Itumbiara & -49.21 & -18.42 \\
\hline Lutzomyia longipalpis & GO & Uruaçu & -49.14 & -14.52 \\
\hline Lutzomyia longipalpis & $\mathrm{DF}$ & Brasília & -47.42 & -15.78 \\
\hline Micropygomyia villelai & MT & Cuiabá & -56.10 & -15.60 \\
\hline Micropygomyia villelai & MT & Barra do Garças & -52.26 & -15.89 \\
\hline Micropygomyia villelai & MT & Alto do Paraguai & -56.48 & -14.51 \\
\hline Micropygomyia villelai & MT & Araguaina & -51.83 & -15.73 \\
\hline Micropygomyia villelai & MT & Arenápolis & -56.82 & -14.43 \\
\hline Micropygomyia villelai & MT & Itiquira & -54.14 & -17.21 \\
\hline Micropygomyia villelai & MT & Juína & -58.74 & -11.38 \\
\hline Micropygomyia villelai & MT & Nobres & -56.33 & -14.73 \\
\hline
\end{tabular}




\begin{tabular}{|c|c|c|c|c|}
\hline Sandfly species & State code & Municipalities & Longitude & Latitude \\
\hline Micropygomyia villelai & MT & Nossa S. do Livramento & -56.37 & -15.80 \\
\hline Micropygomyia villelai & MT & Nova Bandeirantes & -57.86 & -9.95 \\
\hline Micropygomyia villelai & MT & Primavera do Leste & -54.30 & -15.56 \\
\hline Micropygomyia villelai & MT & Rondonópolis & -54.64 & -16.47 \\
\hline Micropygomyia villelai & MT & Rosário Oeste & -56.43 & -14.84 \\
\hline Micropygomyia villelai & MT & Santo Antônio do Leverger & -56.08 & -15.87 \\
\hline Micropygomyia villelai & MT & Vera & -55.19 & -12.18 \\
\hline Micropygomyia villelai & MT & Vila Bela de S. Trindade & -59.95 & -15.00 \\
\hline Micropygomyia villelai & MS & Aquidauana & -55.79 & -20.47 \\
\hline Micropygomyia villelai & MS & Corumbá & -57.65 & -19.01 \\
\hline Micropygomyia villelai & $\mathrm{GO}$ & Campos Verdes & -49.66 & -14.26 \\
\hline Micropygomyia villelai & $\mathrm{GO}$ & Minaçu & -48.22 & -13.53 \\
\hline Micropygomyia villelai & $\mathrm{GO}$ & Porangatu & -49.15 & -13.44 \\
\hline Micropygomyia villelai & $\mathrm{GO}$ & Rio Verde & -50.93 & -17.80 \\
\hline Micropygomyia villelai & GO & Sítio d' Abadia & -46.25 & -14.80 \\
\hline Micropygomyia villelai & $\mathrm{GO}$ & Amaro Leite & -49.15 & -13.97 \\
\hline Micropygomyia villelai & $\mathrm{GO}$ & Cavalcante & -47.50 & -13.80 \\
\hline Micropygomyia longipennis & MT & Varzea Grande & -56.13 & -15.65 \\
\hline Micropygomyia longipennis & MT & Nova Mutum & -56.08 & -13.83 \\
\hline Micropygomyia longipennis & MT & Barra do Garças & -52.26 & -15.89 \\
\hline Micropygomyia longipennis & MT & Alta Floresta & -56.09 & -9.88 \\
\hline Micropygomyia longipennis & MT & Alto Araguaia & -53.22 & -17.31 \\
\hline Micropygomyia longipennis & MT & Alto do Paraguai & -56.48 & -14.51 \\
\hline Micropygomyia longipennis & MT & Apiacás & -57.45 & -9.54 \\
\hline Micropygomyia longipennis & MT & Araguaina & -53.08 & -16.82 \\
\hline Micropygomyia longipennis & MT & Arenápolis & -56.82 & -14.43 \\
\hline Micropygomyia longipennis & MT & Barão do Melgaço & -55.97 & -16.19 \\
\hline Micropygomyia longipennis & MT & Barra dos Bugres & -57.18 & -15.08 \\
\hline Micropygomyia longipennis & MT & Campinápolis & -52.88 & -14.50 \\
\hline Micropygomyia longipennis & MT & Canabrava do Norte & -51.83 & -11.03 \\
\hline Micropygomyia longipennis & MT & Chapada dos Guimarães & -55.75 & -15.46 \\
\hline Micropygomyia longipennis & MT & Confresa & -51.56 & -10.64 \\
\hline Micropygomyia longipennis & MT & Diamantino & -56.45 & -14.41 \\
\hline Micropygomyia longipennis & MT & Feliz Natal & -54.92 & -12.39 \\
\hline Micropygomyia longipennis & MT & Gerneral Carneiro & -52.76 & -15.71 \\
\hline Micropygomyia longipennis & MT & Jaciara & -54.95 & -15.92 \\
\hline Micropygomyia longipennis & MT & Juara & -57.52 & -11.55 \\
\hline Micropygomyia longipennis & MT & Juína & -58.74 & -11.38 \\
\hline Micropygomyia longipennis & MT & Juscimeira & -54.88 & -16.05 \\
\hline Micropygomyia longipennis & MT & Lucas do Rio Verde & -55.91 & -13.05 \\
\hline Micropygomyia longipennis & MT & Luciara & -50.57 & -10.75 \\
\hline Micropygomyia longipennis & MT & Nobres & -56.33 & -14.73 \\
\hline Micropygomyia longipennis & MT & Nova Bandeirantes & -57.86 & -9.95 \\
\hline Micropygomyia longipennis & MT & Nova Ubiratã & -55.26 & -13.01 \\
\hline Micropygomyia longipennis & MT & Novo Horizonte do Norte & -57.35 & -11.41 \\
\hline Micropygomyia longipennis & MT & Paratinga & -54.05 & -14.43 \\
\hline Micropygomyia longipennis & MT & Peixoto de Azevedo & -54.98 & -10.22 \\
\hline Micropygomyia longipennis & MT & Planalto da Serra & -55.27 & -10.44 \\
\hline Micropygomyia longipennis & MT & Poconé & -56.62 & -16.26 \\
\hline Micropygomyia longipennis & MT & Pontal do Araguaia & -52.01 & -15.85 \\
\hline Micropygomyia longipennis & MT & Porto Alegre do Norte & -51.63 & -10.87 \\
\hline Micropygomyia longipennis & MT & Porto dos Gaúchos & -57.41 & -11.52 \\
\hline Micropygomyia longipennis & MT & Porto Esperidião & -58.47 & -15.85 \\
\hline
\end{tabular}




\begin{tabular}{|c|c|c|c|c|}
\hline Sandfly species & State code & Municipalities & Longitude & Latitude \\
\hline Micropygomyia longipennis & MT & Poxoréo & -54.38 & -15.83 \\
\hline Micropygomyia longipennis & MT & Primavera do Leste & -54.30 & -15.56 \\
\hline Micropygomyia longipennis & MT & Rondonópolis & -54.64 & -16.47 \\
\hline Micropygomyia longipennis & MT & Rosário Oeste & -56.43 & -14.84 \\
\hline Micropygomyia longipennis & MT & Santa Carmem & -54.52 & -11.35 \\
\hline Micropygomyia longipennis & MT & São José dos 4 Marcos & -58.17 & -15.17 \\
\hline Micropygomyia longipennis & MT & Sinop & -55.49 & -11.83 \\
\hline Micropygomyia longipennis & MT & Sorriso & -55.71 & -12.55 \\
\hline Micropygomyia longipennis & MT & Taboporã & -56.62 & -10.80 \\
\hline Micropygomyia longipennis & MT & Tapurah & -56.50 & -12.53 \\
\hline Micropygomyia longipennis & MT & União do Sul & -54.36 & -11.52 \\
\hline Micropygomyia longipennis & MT & Vila Rica & -51.11 & -10.01 \\
\hline Micropygomyia longipennis & MT & Ipiranga do Norte & -56.05 & -15.35 \\
\hline Micropygomyia longipennis & MS & Aquidauana & -55.79 & -20.47 \\
\hline Micropygomyia longipennis & MS & Campo Grande & -54.65 & -20.44 \\
\hline Micropygomyia longipennis & MS & Camapuã & -54.04 & -19.53 \\
\hline Micropygomyia longipennis & MS & Corguinho & -54.83 & -19.83 \\
\hline Micropygomyia longipennis & MS & Itaquiraí & -54.11 & -23.28 \\
\hline Micropygomyia longipennis & GO & Sítio d' Abadia & -46.25 & -14.80 \\
\hline Micropygomyia longipennis & $\mathrm{GO}$ & Amaro Leite & -49.15 & -13.97 \\
\hline Micropygomyia longipennis & $\mathrm{GO}$ & Corumbá de Goiás & -48.80 & -15.92 \\
\hline Micropygomyia longipennis & $\mathrm{GO}$ & Ceres & -49.59 & -15.30 \\
\hline Micropygomyia longipennis & $\mathrm{GO}$ & Goiania & -49.25 & -16.67 \\
\hline Micropygomyia longipennis & $\mathrm{GO}$ & Rianópolis & -49.47 & -15.48 \\
\hline Micropygomyia longipennis & $\mathrm{GO}$ & Uruaçú & -49.14 & -14.52 \\
\hline Micropygomyia longipennis & DF & Brasília & -47.42 & -15.78 \\
\hline Micropygomyia quinquefer & MT & Barra dos Bugres & -57.18 & -15.08 \\
\hline Micropygomyia quinquefer & MT & Alto Araguaia & -53.22 & -17.31 \\
\hline Micropygomyia quinquefer & MT & Cáceres & -57.68 & -16.07 \\
\hline Micropygomyia quinquefer & MT & Chapada dos Guimarães & -55.75 & -15.46 \\
\hline Micropygomyia quinquefer & MT & Gaúcha do Norte & -53.08 & -13.24 \\
\hline Micropygomyia quinquefer & MT & Jaciara & -54.95 & -15.92 \\
\hline Micropygomyia quinquefer & MT & Juína & -58.74 & -11.38 \\
\hline Micropygomyia quinquefer & MT & Nova Brasilândia & -54.97 & -14.96 \\
\hline Micropygomyia quinquefer & MT & Pontes de Lacerda & -59.37 & -15.20 \\
\hline Micropygomyia quinquefer & MT & Primavera do Leste & -54.30 & -15.56 \\
\hline Micropygomyia quinquefer & MT & Sorriso & -55.71 & -12.55 \\
\hline Micropygomyia quinquefer & $\mathrm{GO}$ & Itumbiara & -49.22 & -18.42 \\
\hline Micropygomyia quinquefer & $\mathrm{GO}$ & Sítio d'Abadia & -46.25 & -14.80 \\
\hline Micropygomyia quinquefer & $\mathrm{GO}$ & Amaro Leite & -49.15 & -13.97 \\
\hline Micropygomyia quinquefer & MS & Água Clara & -52.87 & -20.44 \\
\hline Micropygomyia quinquefer & MS & Alcinópolis & -53.70 & -18.31 \\
\hline Micropygomyia quinquefer & MS & Aquidauana & -55.79 & -20.47 \\
\hline Micropygomyia quinquefer & MS & Camapuã & -54.04 & -19.53 \\
\hline Micropygomyia quinquefer & MS & Campo Grande & -54.65 & -20.44 \\
\hline Micropygomyia quinquefer & MS & Corguinho & -54.83 & -19.83 \\
\hline Micropygomyia quinquefer & MS & Bonito & -56.47 & -21.13 \\
\hline Micropygomyia quinquefer & MS & Miranda & -56.38 & -20.24 \\
\hline Micropygomyia acanthopharynx & MT & Diamantino & -56.45 & -14.41 \\
\hline Micropygomyia acanthopharynx & MT & Peixoto de Azevedo & -54.98 & -10.22 \\
\hline Micropygomyia acanthopharynx & MT & Barra do Garças & -52.26 & -15.89 \\
\hline Micropygomyia acanthopharynx & MT & Água Boa & -52.16 & -14.05 \\
\hline Micropygomyia acanthopharynx & MT & Alto do Paraguai & -56.48 & -14.51 \\
\hline
\end{tabular}




\begin{tabular}{|c|c|c|c|c|}
\hline Sandfly species & State code & Municipalities & Longitude & Latitude \\
\hline Micropygomyia acanthopharynx & MT & Araguaina & -51.83 & -15.73 \\
\hline Micropygomyia acanthopharynx & MT & Araguainha & -53.08 & -16.82 \\
\hline Micropygomyia acanthopharynx & MT & Aripuanã & -60.63 & -9.17 \\
\hline Micropygomyia acanthopharynx & MT & Campinápolis & -52.88 & -14.50 \\
\hline Micropygomyia acanthopharynx & MT & Canabrava do Norte & -51.83 & -11.03 \\
\hline Micropygomyia acanthopharynx & MT & Canarana & -52.27 & -13.55 \\
\hline Micropygomyia acanthopharynx & MT & Chapada dos Guimarães & -55.75 & -15.46 \\
\hline Micropygomyia acanthopharynx & MT & Cotriguaçu & -58.41 & -9.85 \\
\hline Micropygomyia acanthopharynx & MT & Cuiabá & -56.10 & -15.60 \\
\hline Micropygomyia acanthopharynx & MT & Gaúcha do Norte & -53.08 & -13.24 \\
\hline Micropygomyia acanthopharynx & MT & Jaciara & -54.95 & -15.92 \\
\hline Micropygomyia acanthopharynx & MT & Juína & -58.74 & -11.38 \\
\hline Micropygomyia acanthopharynx & MT & Mercelândia & -54.60 & -11.11 \\
\hline Micropygomyia acanthopharynx & MT & Nobres & -56.33 & -14.73 \\
\hline Micropygomyia acanthopharynx & MT & Nossa S. do Livramento & -56.37 & -15.80 \\
\hline Micropygomyia acanthopharynx & MT & Nova Bandeirantes & -57.86 & -9.95 \\
\hline Micropygomyia acanthopharynx & MT & Nova Brasilândia & -54.57 & -14.57 \\
\hline Micropygomyia acanthopharynx & MT & Nova Santana Helena & -53.35 & -10.82 \\
\hline Micropygomyia acanthopharynx & MT & Novo São Joaquim & -53.02 & -14.90 \\
\hline Micropygomyia acanthopharynx & MT & Paranaíta & -56.48 & -9.67 \\
\hline Micropygomyia acanthopharynx & MT & Paratinga & -54.05 & -14.43 \\
\hline Micropygomyia acanthopharynx & MT & Planalto da Serra & -55.27 & -10.44 \\
\hline Micropygomyia acanthopharynx & MT & Poconé & -56.62 & -16.26 \\
\hline Micropygomyia acanthopharynx & MT & Ponte Branca & -56.78 & -16.08 \\
\hline Micropygomyia acanthopharynx & MT & Porto Esperidião & -58.47 & -15.85 \\
\hline Micropygomyia acanthopharynx & MT & Ribeirão Cascalheira & -51.82 & -12.92 \\
\hline Micropygomyia acanthopharynx & MT & Ribeirãozinho & -52.67 & -16.45 \\
\hline Micropygomyia acanthopharynx & MT & Rondonópolis & -54.64 & -16.47 \\
\hline Micropygomyia acanthopharynx & MT & Rosário Oeste & -56.43 & -14.84 \\
\hline Micropygomyia acanthopharynx & MT & Santo Antônio do Leverger & -56.08 & -15.87 \\
\hline Micropygomyia acanthopharynx & MT & Terra nova do Norte & -55.23 & -10.51 \\
\hline Micropygomyia acanthopharynx & MT & Várzea Grande & -56.13 & -15.65 \\
\hline Micropygomyia acanthopharynx & $\mathrm{GO}$ & Itumbiara & -49.21 & -18.42 \\
\hline Micropygomyia acanthopharynx & Go & Amaro Leite & -49.15 & -13.97 \\
\hline Micropygomyia acanthopharynx & Go & Formoso & -47.33 & -15.53 \\
\hline Micropygomyia acanthopharynx & $\mathrm{GO}$ & Paraúna & -50.44 & -16.94 \\
\hline Micropygomyia acanthopharynx & $\mathrm{GO}$ & Caldas Novas & -48.62 & -17.74 \\
\hline Micropygomyia acanthopharynx & DF & Brasília & -47.42 & -15.78 \\
\hline Micropygomyia acanthopharynx & MS & Aquidauana & -55.79 & -20.47 \\
\hline Micropygomyia acanthopharynx & MS & Corguinho & -54.83 & -19.83 \\
\hline Micropygomyia acanthopharynx & MS & Rio Negro & -54.99 & -19.45 \\
\hline Micropygomyia acanthopharynx & MS & Bonito & -56.47 & -21.13 \\
\hline Micropygomyia acanthopharynx & MS & Camapuã & -54.04 & -19.53 \\
\hline Micropygomyia acanthopharynx & MS & Rio Verde de Mato Grosso & -54.84 & -18.92 \\
\hline Migonemyia migonei & MT & Peixoto de Azevedo & -54.98 & -10.22 \\
\hline Migonemyia migonei & MT & Apiacás & -57.45 & -9.54 \\
\hline Migonemyia migonei & MT & Aripuanã & -60.63 & -9.17 \\
\hline Migonemyia migonei & MT & Claúdia & -54.88 & -11.49 \\
\hline Migonemyia migonei & MT & Comodoro & -59.79 & -13.66 \\
\hline Migonemyia migonei & MT & Feliz Natal & -54.92 & -12.39 \\
\hline Migonemyia migonei & MT & Guarantã do Norte & -54.90 & -9.78 \\
\hline Migonemyia migonei & MT & Guiratinga & -53.74 & -16.33 \\
\hline Migonemyia migonei & MT & Juína & -58.74 & -11.38 \\
\hline
\end{tabular}




\begin{tabular}{|c|c|c|c|c|}
\hline Sandfly species & State code & Municipalities & Longitude & Latitude \\
\hline Migonemyia migonei & MT & Lucas do Rio Verde & -55.91 & -13.05 \\
\hline Migonemyia migonei & MT & Mercelândia & -54.60 & -11.11 \\
\hline Migonemyia migonei & MT & Nova Guarita & -55.40 & -10.30 \\
\hline Migonemyia migonei & MT & Nova Ubiratã & -55.26 & -13.01 \\
\hline Migonemyia migonei & MT & Paranaíta & -56.48 & -9.67 \\
\hline Migonemyia migonei & MT & Sinop & -55.49 & -11.83 \\
\hline Migonemyia migonei & MT & Sorriso & -55.71 & -12.55 \\
\hline Migonemyia migonei & MT & Tangará da Serra & -57.50 & -14.62 \\
\hline Migonemyia migonei & MT & Terra nova do Norte & -55.23 & -10.51 \\
\hline Migonemyia migonei & MT & União do Sul & -54.36 & -11.52 \\
\hline Migonemyia migonei & MT & Ipiranga do Norte & -56.05 & -15.35 \\
\hline Migonemyia migonei & $\mathrm{GO}$ & Maurilândia & -50.31 & -18.00 \\
\hline Migonemyia migonei & $\mathrm{GO}$ & Niquelândia & -48.45 & -14.47 \\
\hline Migonemyia migonei & $\mathrm{GO}$ & Silvânia & -48.61 & -16.66 \\
\hline Migonemyia migonei & $\mathrm{GO}$ & Uriapuru & -49.92 & -14.29 \\
\hline Migonemyia migonei & MS & Antonio João & -55.95 & -22.19 \\
\hline Migonemyia migonei & MS & Bodoquena & -56.72 & -20.54 \\
\hline Migonemyia migonei & MS & Campo Grande & -54.65 & -20.44 \\
\hline Migonemyia migonei & MS & Dourados & -54.81 & -22.22 \\
\hline Migonemyia migonei & MS & Jardim & -56.14 & -21.48 \\
\hline Migonemyia migonei & MS & Rio Brilhante & -54.54 & -21.80 \\
\hline Migonemyia migonei & MS & Rio Verde de Mato Grosso & -54.84 & -18.92 \\
\hline Migonemyia migonei & MS & Bataguassu & -52.42 & -21.71 \\
\hline Migonemyia migonei & MS & Caarapó & -54.80 & -22.63 \\
\hline Nyssomyia whitmani & MT & Claúdia & -54.88 & -11.49 \\
\hline Nyssomyia whitmani & MT & Confresa & -51.56 & -10.64 \\
\hline Nyssomyia whitmani & MT & Canabrava do Norte & -51.83 & -11.03 \\
\hline Nyssomyia whitmani & MT & Chapada dos Guimarães & -55.75 & -15.46 \\
\hline Nyssomyia whitmani & MT & Cláudia & -54.88 & -11.49 \\
\hline Nyssomyia whitmani & MT & Feliz Natal & -54.92 & -12.39 \\
\hline Nyssomyia whitmani & MT & Jangada & -56.49 & -15.24 \\
\hline Nyssomyia whitmani & MT & Itiquira & -54.14 & -17.21 \\
\hline Nyssomyia whitmani & MT & Várzea Grande & -56.13 & -15.65 \\
\hline Nyssomyia whitmani & MT & Nova Mutum & -56.08 & -13.83 \\
\hline Nyssomyia whitmani & MT & Novo São Joaquim & -53.02 & -14.90 \\
\hline Nyssomyia whitmani & MT & Nova Brasilândia & -54.57 & -14.57 \\
\hline Nyssomyia whitmani & MT & Nova Bandeirantes & -57.86 & -9.95 \\
\hline Nyssomyia whitmani & MT & Nortelândia & -56.80 & -14.42 \\
\hline Nyssomyia whitmani & MT & Nova Guarita & -55.40 & -10.30 \\
\hline Nyssomyia whitmani & MT & Nova Ubiratã & -55.26 & -13.01 \\
\hline Nyssomyia whitmani & MT & Novo Horizonte do Norte & -57.35 & -11.41 \\
\hline Nyssomyia whitmani & MT & Nova Canaã do Norte & -55.95 & -10.55 \\
\hline Nyssomyia whitmani & MT & Nova Maringá & -57.06 & -13.01 \\
\hline Nyssomyia whitmani & MT & Nossa S. do Livramento & -56.37 & -15.80 \\
\hline Nyssomyia whitmani & MT & Nova Santa Helena & -53.35 & -10.82 \\
\hline Nyssomyia whitmani & MT & Nova Xavantina & -52.34 & -14.66 \\
\hline Nyssomyia whitmani & MT & Nobres & -56.33 & -14.73 \\
\hline Nyssomyia whitmani & MT & Juscimeira & -54.88 & -16.05 \\
\hline Nyssomyia whitmani & MT & Juara & -57.52 & -11.55 \\
\hline Nyssomyia whitmani & MT & Jaciara & -54.95 & -15.92 \\
\hline Nyssomyia whitmani & MT & Juína & -58.74 & -11.38 \\
\hline Nyssomyia whitmani & MT & São Pedro da Cipa & -54.91 & -15.99 \\
\hline Nyssomyia whitmani & MT & Santo Antonio do Leste & -53.62 & -14.83 \\
\hline
\end{tabular}




\begin{tabular}{|c|c|c|c|c|}
\hline Sandfly species & State code & Municipalities & Longitude & Latitude \\
\hline Nyssomyia whitmani & MT & Santo Antônio do Leverger & -56.08 & -15.87 \\
\hline Nyssomyia whitmani & MT & Rondonópolis & -54.64 & -16.47 \\
\hline Nyssomyia whitmani & MT & Sinop & -55.49 & -11.83 \\
\hline Nyssomyia whitmani & MT & Sapezal & -57.20 & -14.97 \\
\hline Nyssomyia whitmani & MT & Sorriso & -55.71 & -12.55 \\
\hline Nyssomyia whitmani & MT & Guiratinga & -53.74 & -16.33 \\
\hline Nyssomyia whitmani & MT & Gaúcha do Norte & -53.08 & -13.24 \\
\hline Nyssomyia whitmani & MT & Guarantã do Norte & -54.90 & -9.78 \\
\hline Nyssomyia whitmani & MT & Matupá & -54.90 & -10.05 \\
\hline Nyssomyia whitmani & MT & Marcelândia & -54.60 & -11.11 \\
\hline Nyssomyia whitmani & MT & General Carneiro & -52.76 & -15.71 \\
\hline Nyssomyia whitmani & MT & Planalto da Serra & -55.27 & -10.44 \\
\hline Nyssomyia whitmani & MT & Pontes de Lacerda & -59.37 & -15.20 \\
\hline Nyssomyia whitmani & MT & Paranatinga & -54.05 & -14.43 \\
\hline Nyssomyia whitmani & MT & Nova Brasilândia & -54.97 & -14.96 \\
\hline Nyssomyia whitmani & MT & Tambaporã & -56.62 & -10.80 \\
\hline Nyssomyia whitmani & MT & Tangará da Serra & -57.50 & -14.62 \\
\hline Nyssomyia whitmani & MT & Tapurah & -56.50 & -12.53 \\
\hline Nyssomyia whitmani & MT & Serra Nova Dourada & -51.58 & -11.97 \\
\hline Nyssomyia whitmani & MT & São José dos 4 Marcos & -58.17 & -15.17 \\
\hline Nyssomyia whitmani & MT & São José do Povo & -54.25 & -16.46 \\
\hline Nyssomyia whitmani & MT & São Félix do Araguaia & -50.67 & -11.61 \\
\hline Nyssomyia whitmani & MT & Paranaíta & -56.48 & -9.67 \\
\hline Nyssomyia whitmani & MT & Porto dos Gaúchos & -57.41 & -11.52 \\
\hline Nyssomyia whitmani & MT & Porto Esperidião & -58.47 & -15.85 \\
\hline Nyssomyia whitmani & MT & Santa Rita do Trivelato & -55.26 & -13.81 \\
\hline Nyssomyia whitmani & MT & Vila Rica & -51.11 & -10.01 \\
\hline Nyssomyia whitmani & MT & Vila Bela da S. Trindade & -59.95 & -15.00 \\
\hline Nyssomyia whitmani & MT & Itanguá & -56.64 & -12.23 \\
\hline Nyssomyia whitmani & MT & União do Sul & -54.36 & -11.52 \\
\hline Nyssomyia whitmani & MT & Ribeirão Cascalheira & -51.82 & -12.92 \\
\hline Nyssomyia whitmani & MT & Ribeirãozinho & -52.67 & -16.45 \\
\hline Nyssomyia whitmani & MT & Rosário D' Oeste & -56.43 & -14.84 \\
\hline Nyssomyia whitmani & MT & Pontal do Araguaia & -52.01 & -15.85 \\
\hline Nyssomyia whitmani & MT & Peixoto de Azevedo & -54.98 & -10.22 \\
\hline Nyssomyia whitmani & MT & Pedra Preta & -54.47 & -16.62 \\
\hline Nyssomyia whitmani & MT & Ponte Branca & -56.78 & -16.08 \\
\hline Nyssomyia whitmani & MT & Porto Alegre do Norte & -51.63 & -10.87 \\
\hline Nyssomyia whitmani & MT & Vera & -55.31 & -12.30 \\
\hline Nyssomyia whitmani & MT & Primavera do Leste & -54.30 & -15.56 \\
\hline Nyssomyia whitmani & MT & Pontal do Araguaia & -52.00 & -15.84 \\
\hline Nyssomyia whitmani & MT & Poconé & -56.62 & -16.26 \\
\hline Nyssomyia whitmani & MT & Terra Nova do Norte & -55.23 & -10.51 \\
\hline Nyssomyia whitmani & MT & Lucas do Rio Verde & -55.91 & -13.05 \\
\hline Nyssomyia whitmani & MT & Luciara & -50.57 & -10.75 \\
\hline Nyssomyia whitmani & MT & Barra dos Bugres & -57.18 & -15.08 \\
\hline Nyssomyia whitmani & MT & Brasnorte & -57.98 & -12.15 \\
\hline Nyssomyia whitmani & MT & Barra do Garças & -52.26 & -15.89 \\
\hline Nyssomyia whitmani & MT & Barão de Melgaço & -55.97 & -16.19 \\
\hline Nyssomyia whitmani & MT & Poxoréo & -54.38 & -15.83 \\
\hline Nyssomyia whitmani & MT & Araguaiana & -51.83 & -15.73 \\
\hline Nyssomyia whitmani & MT & Araguainha & -53.08 & -16.82 \\
\hline Nyssomyia whitmani & MT & Cuiabá & -56.10 & -15.60 \\
\hline
\end{tabular}




\begin{tabular}{|c|c|c|c|c|}
\hline Sandfly species & State code & Municipalities & Longitude & Latitude \\
\hline Nyssomyia whitmani & MT & Cáceres & -57.68 & -16.07 \\
\hline Nyssomyia whitmani & MT & Campinápolis & -52.88 & -14.50 \\
\hline Nyssomyia whitmani & MS & Amambai & -55.22 & -23.08 \\
\hline Nyssomyia whitmani & MS & Anastácio & -55.81 & -20.48 \\
\hline Nyssomyia whitmani & MS & Antonio João & -55.95 & -22.19 \\
\hline Nyssomyia whitmani & MS & Alcinópolis & -53.70 & -18.31 \\
\hline Nyssomyia whitmani & MS & Aparecida do Taboado & -51.09 & -20.09 \\
\hline Nyssomyia whitmani & MS & Aquidauana & -55.79 & -20.47 \\
\hline Nyssomyia whitmani & MS & Bela Vista & -56.52 & -22.11 \\
\hline Nyssomyia whitmani & MS & Bataguassú & -52.42 & -21.71 \\
\hline Nyssomyia whitmani & MS & Bodoquena & -56.72 & -20.54 \\
\hline Nyssomyia whitmani & MS & Brasilândia & -52.04 & -21.26 \\
\hline Nyssomyia whitmani & MS & Camapuã & -54.04 & -19.53 \\
\hline Nyssomyia whitmani & MS & Campo Grande & -54.65 & -20.44 \\
\hline Nyssomyia whitmani & MS & Caarapó & -54.80 & -22.63 \\
\hline Nyssomyia whitmani & MS & Corguinho & -54.83 & -19.83 \\
\hline Nyssomyia whitmani & MS & Caracol & -57.02 & -22.01 \\
\hline Nyssomyia whitmani & MS & Coxim & -54.76 & -18.51 \\
\hline Nyssomyia whitmani & MS & Ponta Porã & -55.73 & -22.54 \\
\hline Nyssomyia whitmani & MS & Porto Murtinho & -57.88 & -21.70 \\
\hline Nyssomyia whitmani & MS & Ribas do Rio Pardo & -53.76 & -20.44 \\
\hline Nyssomyia whitmani & MS & Rio Negro & -54.99 & -19.45 \\
\hline Nyssomyia whitmani & MS & Rochedo & -54.89 & -19.95 \\
\hline Nyssomyia whitmani & MS & São Gabriel d' Oeste & -54.57 & -19.40 \\
\hline Nyssomyia whitmani & MS & Selvíria & -51.42 & -20.37 \\
\hline Nyssomyia whitmani & MS & Sidrolândia & -54.96 & -20.93 \\
\hline Nyssomyia whitmani & MS & Terenos & -54.86 & -20.44 \\
\hline Nyssomyia whitmani & MS & Três Lagoas & -51.68 & -20.75 \\
\hline Nyssomyia whitmani & MS & Costa Rica & -53.13 & -18.54 \\
\hline Nyssomyia whitmani & MS & Dourados & -54.81 & -22.22 \\
\hline Nyssomyia whitmani & MS & Guia Lopes da Laguna & -56.11 & -21.46 \\
\hline Nyssomyia whitmani & MS & Inocência & -51.93 & -19.73 \\
\hline Nyssomyia whitmani & MS & Jaraguarí & -54.40 & -20.14 \\
\hline Nyssomyia whitmani & MS & Jardim & -56.14 & -21.48 \\
\hline Nyssomyia whitmani & MS & Nhecolândia & -57.07 & -19.27 \\
\hline Nyssomyia whitmani & MS & Novo Horizonte do Sul & -53.80 & -22.61 \\
\hline Nyssomyia whitmani & MS & Nova Alvorada Do Sul & -54.38 & -21.47 \\
\hline Nyssomyia whitmani & MS & Laguna Carapã & -55.02 & -22.45 \\
\hline Nyssomyia whitmani & MS & Naviraí & -54.22 & -23.13 \\
\hline Nyssomyia whitmani & MS & Nioaque & -55.83 & -21.14 \\
\hline Nyssomyia whitmani & MS & Santa Rita do Pardo & -52.83 & -21.30 \\
\hline Nyssomyia whitmani & MS & Bonito & -56.47 & -21.13 \\
\hline Nyssomyia whitmani & MS & Dois Irmãos do Buriti & -55.30 & -20.68 \\
\hline Nyssomyia whitmani & MS & Maracajú & -55.17 & -21.61 \\
\hline Nyssomyia whitmani & MS & Miranda & -56.38 & -20.24 \\
\hline Nyssomyia whitmani & MS & Pedro Gomes & -54.55 & -18.10 \\
\hline Nyssomyia whitmani & MS & Paranhos & -55.43 & -23.89 \\
\hline Nyssomyia whitmani & MS & Rio Brilhante & -54.54 & -21.80 \\
\hline Nyssomyia whitmani & MS & Rio Verde de Mato Grosso & -54.84 & -18.92 \\
\hline Nyssomyia whitmani & $\mathrm{GO}$ & Anápolis & -48.95 & -16.32 \\
\hline Nyssomyia whitmani & $\mathrm{GO}$ & Aragarças & -52.25 & -15.92 \\
\hline Nyssomyia whitmani & $\mathrm{GO}$ & Araguapaz & -50.63 & -15.09 \\
\hline Nyssomyia whitmani & GO & Bela Vista & -48.95 & -16.97 \\
\hline
\end{tabular}




\begin{tabular}{|c|c|c|c|c|}
\hline Sandfly species & State code & Municipalities & Longitude & Latitude \\
\hline Nyssomyia whitmani & $\mathrm{GO}$ & Carmo do Rio Verde & -49.70 & -15.35 \\
\hline Nyssomyia whitmani & $\mathrm{GO}$ & Campos Verdes & -49.66 & -14.26 \\
\hline Nyssomyia whitmani & $\mathrm{GO}$ & Campestre & -49.69 & -16.73 \\
\hline Nyssomyia whitmani & $\mathrm{GO}$ & Colinas do Sul & -48.08 & -14.15 \\
\hline Nyssomyia whitmani & $\mathrm{GO}$ & Formosa & -47.33 & -15.53 \\
\hline Nyssomyia whitmani & $\mathrm{GO}$ & Israelândia & -50.90 & -16.35 \\
\hline Nyssomyia whitmani & $\mathrm{GO}$ & Padre Bernado & -48.50 & -13.35 \\
\hline Nyssomyia whitmani & $\mathrm{GO}$ & Doverlândia & -52.31 & -16.69 \\
\hline Nyssomyia whitmani & $\mathrm{GO}$ & Crixás & -49.97 & -14.45 \\
\hline Nyssomyia whitmani & $\mathrm{GO}$ & Cumari & -48.15 & -18.26 \\
\hline Nyssomyia whitmani & $\mathrm{GO}$ & Catalão & -47.95 & -18.17 \\
\hline Nyssomyia whitmani & $\mathrm{GO}$ & Colinas do Sul & -48.07 & -14.14 \\
\hline Nyssomyia whitmani & $\mathrm{GO}$ & Carmo do Rio Verde & -49.70 & -15.35 \\
\hline Nyssomyia whitmani & $\mathrm{GO}$ & Goiás & -50.14 & -15.93 \\
\hline Nyssomyia whitmani & $\mathrm{GO}$ & Goiatuba & -49.37 & -18.02 \\
\hline Nyssomyia whitmani & $\mathrm{GO}$ & Itumbiara & -49.22 & -18.42 \\
\hline Nyssomyia whitmani & $\mathrm{GO}$ & Cavalcante & -47.50 & -13.80 \\
\hline Nyssomyia whitmani & $\mathrm{GO}$ & Jataí & -51.72 & -17.88 \\
\hline Nyssomyia whitmani & $\mathrm{GO}$ & Guapó & -49.53 & -16.83 \\
\hline Nyssomyia whitmani & $\mathrm{GO}$ & Mineiros & -50.73 & -16.54 \\
\hline Nyssomyia whitmani & $\mathrm{GO}$ & Mambaí & -46.11 & -14.49 \\
\hline Nyssomyia whitmani & $\mathrm{GO}$ & Maurilândia & -50.31 & -18.00 \\
\hline Nyssomyia whitmani & $\mathrm{GO}$ & Montividiu do Norte & -48.61 & -13.11 \\
\hline Nyssomyia whitmani & $\mathrm{GO}$ & Mutunópolis & -49.28 & -13.73 \\
\hline Nyssomyia whitmani & $\mathrm{GO}$ & Mundo Novo & -40.47 & -11.86 \\
\hline Nyssomyia whitmani & $\mathrm{GO}$ & Minaçu & -48.22 & -13.53 \\
\hline Nyssomyia whitmani & $\mathrm{GO}$ & Morro Agudo & -50.06 & -15.32 \\
\hline Nyssomyia whitmani & $\mathrm{GO}$ & Niquelândia & -48.46 & -14.47 \\
\hline Nyssomyia whitmani & $\mathrm{GO}$ & Nova Crixás & -50.33 & -14.10 \\
\hline Nyssomyia whitmani & $\mathrm{GO}$ & Porangatu & -49.15 & -13.44 \\
\hline Nyssomyia whitmani & $\mathrm{GO}$ & Posse & -46.37 & -14.09 \\
\hline Nyssomyia whitmani & $\mathrm{GO}$ & Rianópolis & -49.47 & -15.48 \\
\hline Nyssomyia whitmani & $\mathrm{GO}$ & Quirinópolis & -50.50 & -18.53 \\
\hline Nyssomyia whitmani & $\mathrm{GO}$ & Rio Verde & -50.93 & -17.80 \\
\hline Nyssomyia whitmani & $\mathrm{GO}$ & São Domingos & -46.32 & -13.40 \\
\hline Nyssomyia whitmani & $\mathrm{GO}$ & São João d'Aliança & -47.79 & -14.88 \\
\hline Nyssomyia whitmani & $\mathrm{GO}$ & Sítio d'Abadia & -46.25 & -14.80 \\
\hline Nyssomyia whitmani & $\mathrm{GO}$ & São Miguel da Araguaina & -50.22 & -13.32 \\
\hline Nyssomyia whitmani & $\mathrm{GO}$ & Silvânia & 48.61 & -16.66 \\
\hline Nyssomyia whitmani & $\mathrm{GO}$ & Uriapuru & -49.92 & -14.29 \\
\hline Nyssomyia whitmani & $\mathrm{GO}$ & Uruaçu & -49.14 & -14.52 \\
\hline Nyssomyia whitmani & DF & Brasília & -47.42 & -15.78 \\
\hline Nyssomyia whitmani & MS & Água Clara & -52.87 & -20.44 \\
\hline Nyssomyia neivai & MS & Água Clara & -52.87 & -20.44 \\
\hline Nyssomyia neivai & MS & Aquidauana & -55.79 & -20.47 \\
\hline Nyssomyia neivai & MS & Bataguassu & -52.42 & -21.71 \\
\hline Nyssomyia neivai & MS & Bodoquena & -56.72 & -20.54 \\
\hline Nyssomyia neivai & MS & Brasilândia & -52.04 & -21.26 \\
\hline Nyssomyia neivai & MS & Rochedo & -54.89 & -19.95 \\
\hline Nyssomyia neivai & MS & Terenos & -54.86 & -20.44 \\
\hline Nyssomyia neivai & MS & Três Lagoas & -51.68 & -20.75 \\
\hline Nyssomyia neivai & MS & Dourados & -54.81 & -22.22 \\
\hline Nyssomyia neivai & MS & Guia Lopes da Laguna & -56.11 & -21.46 \\
\hline
\end{tabular}




\begin{tabular}{|c|c|c|c|c|}
\hline Sandfly species & State code & Municipalities & Longitude & Latitude \\
\hline Nyssomyia neivai & MS & Itaquiraí & -54.11 & -23.28 \\
\hline Nyssomyia neivai & MS & Naviraí & -54.22 & -23.13 \\
\hline Nyssomyia neivai & MS & Nova Andradina & -53.34 & -22.23 \\
\hline Nyssomyia neivai & MS & Novo Horizonte do Sul & -53.80 & -22.61 \\
\hline Nyssomyia neivai & MS & Santa Rita do Pardo & -52.83 & -21.30 \\
\hline Nyssomyia neivai & MS & Bonito & -56.47 & -21.13 \\
\hline Nyssomyia neivai & MS & Mundo Novo & -54.27 & -23.94 \\
\hline Nyssomyia neivai & MS & Rio Verde de Mato Grosso & -54.84 & -18.92 \\
\hline Nyssomyia neivai & $\mathrm{GO}$ & Santa Helena de Goiás & -50.58 & -17.72 \\
\hline Nyssomyia neivai & $\mathrm{GO}$ & Corumbaíba & -48.57 & -18.15 \\
\hline Nyssomyia neivai & $\mathrm{GO}$ & Itumbiara & -49.21 & -18.42 \\
\hline Nyssomyia neivai & $\mathrm{DF}$ & Brasília & -47.42 & -15.78 \\
\hline Nyssomyia intermedia & MT & Cuiabá & -56.10 & -15.60 \\
\hline Nyssomyia intermedia & MT & Gaúcha do Norte & -53.08 & -13.24 \\
\hline Nyssomyia intermedia & MT & Rosário do Oeste & -56.42 & -14.83 \\
\hline Nyssomyia intermedia & $\mathrm{GO}$ & Aragarças & -52.25 & 15.92 \\
\hline Nyssomyia intermedia & GO & Araguapaz & -50.63 & -15.09 \\
\hline Nyssomyia intermedia & $\mathrm{GO}$ & Carmo do Rio Verde & -49.70 & -15.35 \\
\hline Nyssomyia intermedia & $\mathrm{GO}$ & Campestre de Goiás & -49.69 & -16.73 \\
\hline Nyssomyia intermedia & $\mathrm{GO}$ & Cumari & -48.15 & -18.26 \\
\hline Nyssomyia intermedia & GO & Catalão & -47.95 & -18.17 \\
\hline Nyssomyia intermedia & $\mathrm{GO}$ & Crixás & -49.97 & -14.45 \\
\hline Nyssomyia intermedia & GO & Flores de Goiás & -47.05 & -14.45 \\
\hline Nyssomyia intermedia & GO & Goiás & -50.14 & -15.93 \\
\hline Nyssomyia intermedia & $\mathrm{GO}$ & Goiatuba & -49.37 & -18.02 \\
\hline Nyssomyia intermedia & GO & Itumbiara & -49.22 & -18.42 \\
\hline Nyssomyia intermedia & GO & Guapó & -49.53 & -16.83 \\
\hline Nyssomyia intermedia & $\mathrm{GO}$ & Mambaí & -46.11 & -14.49 \\
\hline Nyssomyia intermedia & GO & Maurilândia & -50.31 & -18.00 \\
\hline Nyssomyia intermedia & GO & Mutunópolis & -49.28 & -13.73 \\
\hline Nyssomyia intermedia & $\mathrm{GO}$ & Mundo Novo & -40.47 & -11.86 \\
\hline Nyssomyia intermedia & $\mathrm{GO}$ & Minaçu & -48.22 & -13.53 \\
\hline Nyssomyia intermedia & GO & Morro Agudo & -50.06 & -15.32 \\
\hline Nyssomyia intermedia & GO & Niquelândia & -48.46 & -14.47 \\
\hline Nyssomyia intermedia & GO & Posse & -46.37 & -14.09 \\
\hline Nyssomyia intermedia & GO & Quirinópolis & -50.50 & -18.53 \\
\hline Nyssomyia intermedia & GO & São Domingos & -46.32 & -13.40 \\
\hline Nyssomyia intermedia & GO & São Miguel da Araguaina & -50.22 & -13.32 \\
\hline Nyssomyia intermedia & GO & Silvânia & -48.61 & -16.66 \\
\hline Nyssomyia intermedia & GO & Sitio d'Abadia & -46.25 & -14.80 \\
\hline Nyssomyia intermedia & GO & Mineiros & -50.73 & -16.54 \\
\hline Nyssomyia intermedia & $\mathrm{GO}$ & Corumbaíba & -48.56 & -18.14 \\
\hline Nyssomyia intermedia & $\mathrm{GO}$ & Cavalcate & -47.50 & -13.80 \\
\hline Nyssomyia intermedia & GO & Formosa & -47.33 & -15.53 \\
\hline Nyssomyia intermedia & GO & Padre Bernado & -48.50 & -15.35 \\
\hline Nyssomyia intermedia & GO & Pontalina & -49.44 & -17.52 \\
\hline Nyssomyia antunesi & MT & Diamantino & -56.45 & -14.41 \\
\hline Nyssomyia antunesi & MT & Barra dos Bugres & -57.18 & -15.08 \\
\hline Nyssomyia antunesi & MT & Barra do Garças & -52.26 & -15.89 \\
\hline Nyssomyia antunesi & MT & Peixoto de Azevedo & -54.98 & -10.22 \\
\hline Nyssomyia antunesi & MT & Nova Mutum & -56.08 & -13.83 \\
\hline Nyssomyia antunesi & MT & Alto Araguaia & -53.22 & -17.31 \\
\hline Nyssomyia antunesi & MT & Apiacás & -57.45 & -9.54 \\
\hline
\end{tabular}




\begin{tabular}{|c|c|c|c|c|}
\hline Sandfly species & State code & Municipalities & Longitude & Latitude \\
\hline Nyssomyia antunesi & MT & Alta Floresta & -56.09 & -9.88 \\
\hline Nyssomyia antunesi & MT & Alto Taquarí & -53.16 & -17.49 \\
\hline Nyssomyia antunesi & MT & Aripuanã & -60.63 & -9.17 \\
\hline Nyssomyia antunesi & MT & Barão do Melgaço & -55.97 & -16.19 \\
\hline Nyssomyia antunesi & MT & Brasnorte & -57.98 & -12.15 \\
\hline Nyssomyia antunesi & MT & Cáceres & -57.68 & -16.07 \\
\hline Nyssomyia antunesi & MT & Campo Novo do Parecis & -57.88 & -13.67 \\
\hline Nyssomyia antunesi & MT & Campos de Julio & -59.08 & -13.53 \\
\hline Nyssomyia antunesi & MT & Canabrava do Norte & -51.83 & -11.03 \\
\hline Nyssomyia antunesi & MT & Canarana & -52.27 & -13.55 \\
\hline Nyssomyia antunesi & MT & Carlinda & -55.83 & -9.96 \\
\hline Nyssomyia antunesi & MT & Chapada dos Guimarães & -55.75 & -15.46 \\
\hline Nyssomyia antunesi & MT & Claúdia & -54.88 & -11.49 \\
\hline Nyssomyia antunesi & MT & Colíder & -55.45 & -10.81 \\
\hline Nyssomyia antunesi & MT & Colniza & -59.03 & -9.40 \\
\hline Nyssomyia antunesi & MT & Comodoro & -59.79 & -13.66 \\
\hline Nyssomyia antunesi & MT & Cotriguaçu & -58.41 & -9.85 \\
\hline Nyssomyia antunesi & MT & Feliz Natal & -54.92 & -12.39 \\
\hline Nyssomyia antunesi & MT & Gaúcha do Norte & -53.08 & -13.24 \\
\hline Nyssomyia antunesi & MT & Gerneral Carneiro & -52.76 & -15.71 \\
\hline Nyssomyia antunesi & MT & Guarantã do Norte & -54.90 & -9.78 \\
\hline Nyssomyia antunesi & MT & Itiquira & -54.14 & -17.21 \\
\hline Nyssomyia antunesi & MT & Jaciara & -54.95 & -15.92 \\
\hline Nyssomyia antunesi & MT & Juara & -57.52 & -11.55 \\
\hline Nyssomyia antunesi & MT & Juína & -58.74 & -11.38 \\
\hline Nyssomyia antunesi & MT & Lucas do Rio Verde & -55.91 & -13.05 \\
\hline Nyssomyia antunesi & MT & Mercelândia & -54.60 & -11.11 \\
\hline Nyssomyia antunesi & MT & Matupá & -54.90 & -10.05 \\
\hline Nyssomyia antunesi & MT & Nobres & -56.33 & -14.73 \\
\hline Nyssomyia antunesi & MT & Notelândia & -56.80 & -14.42 \\
\hline Nyssomyia antunesi & MT & Nova Bandeirante & -57.86 & -9.95 \\
\hline Nyssomyia antunesi & MT & Nova Canaã do Norte & -55.95 & -10.55 \\
\hline Nyssomyia antunesi & MT & Nova Guarita & -55.40 & -10.30 \\
\hline Nyssomyia antunesi & MT & Nova Lacerda & -59.35 & -14.28 \\
\hline Nyssomyia antunesi & MT & Nova Marilândia & -56.57 & -14.21 \\
\hline Nyssomyia antunesi & MT & Nova Maringá & -57.06 & -13.01 \\
\hline Nyssomyia antunesi & MT & Nova Ubiratã & -55.26 & -13.01 \\
\hline Nyssomyia antunesi & MT & Novo Horizonte do Norte & -57.35 & -11.41 \\
\hline Nyssomyia antunesi & MT & Paranaíta & -56.48 & -9.67 \\
\hline Nyssomyia antunesi & MT & Peixoto de Azevedo & -54.98 & -10.22 \\
\hline Nyssomyia antunesi & MT & Pontes de Lacerda & -59.37 & -15.20 \\
\hline Nyssomyia antunesi & MT & Porto Alegre do Norte & -51.63 & -10.87 \\
\hline Nyssomyia antunesi & MT & Porto dos Gaúchos & -57.41 & -11.52 \\
\hline Nyssomyia antunesi & MT & Porto Esperidião & -58.47 & -15.85 \\
\hline Nyssomyia antunesi & MT & Poxoréo & -54.38 & -15.83 \\
\hline Nyssomyia antunesi & MT & Querência & -52.11 & -12.35 \\
\hline Nyssomyia antunesi & MT & Ribeirãozinho & -52.67 & -16.45 \\
\hline Nyssomyia antunesi & MT & Rondonópolis & -54.64 & -16.47 \\
\hline Nyssomyia antunesi & MT & Santa Rita do Trivelato & -55.26 & -13.81 \\
\hline Nyssomyia antunesi & MT & São Felix do Araguaia & -50.67 & -11.61 \\
\hline Nyssomyia antunesi & MT & São José dos 4 Marcos & -58.17 & -15.17 \\
\hline Nyssomyia antunesi & MT & Sapezal & -57.20 & -14.97 \\
\hline Nyssomyia antunesi & MT & Sorriso & -55.71 & -12.55 \\
\hline
\end{tabular}




\begin{tabular}{|c|c|c|c|c|}
\hline Sandfly species & State code & Municipalities & Longitude & Latitude \\
\hline Nyssomyia antunesi & MT & Tapurah & -56.50 & -12.53 \\
\hline Nyssomyia antunesi & MT & Terra Nova do Norte & -55.23 & -10.51 \\
\hline Nyssomyia antunesi & MT & União do Sul & -54.36 & -11.52 \\
\hline Nyssomyia antunesi & MT & Várzea Grande & -56.13 & -15.65 \\
\hline Nyssomyia antunesi & MT & Vera & -55.19 & -12.18 \\
\hline Nyssomyia antunesi & MT & Vila Bela de S. Trindade & -59.95 & -15.00 \\
\hline Nyssomyia antunesi & MT & Itanhangá & -56.64 & -12.23 \\
\hline Nyssomyia antunesi & MT & Ipiranga do Norte & -56.05 & -15.35 \\
\hline Nyssomyia antunesi & MS & Coxim & -54.76 & -18.51 \\
\hline Nyssomyia antunesi & MS & Rio Verde de Mato Grosso & -54.84 & -18.92 \\
\hline Nyssomyia antunesi & MS & Campo Grande & -54.65 & -20.44 \\
\hline Pintomyia christenseni & MS & Alcinópolis & -53.70 & -18.31 \\
\hline Pintomyia christenseni & MS & Bela Vista & -56.52 & -22.11 \\
\hline Pintomyia christenseni & MS & Campo Grande & -54.65 & -20.44 \\
\hline Pintomyia christenseni & MS & Corguinho & -54.83 & -19.83 \\
\hline Pintomyia christenseni & MS & Novo Horizonte do Sul & -53.80 & -22.61 \\
\hline Pintomyia christenseni & MS & Bonito & -56.47 & -21.13 \\
\hline Pintomyia christenseni & MS & Maracajú & -55.17 & -21.61 \\
\hline Pintomyia christenseni & MS & Paraíso das Águas & -52.97 & -19.05 \\
\hline Pintomyia christenseni & MS & Rio Verde de Mato Grosso & -54.84 & -18.92 \\
\hline Pintomyia christenseni & $\mathrm{GO}$ & Itumbiara & -49.21 & -18.42 \\
\hline Pintomyia christenseni & $\mathrm{GO}$ & Sítio d’ Abadia & -46.25 & -14.80 \\
\hline Pintomyia christenseni & $\mathrm{GO}$ & Amaro Leite & -49.15 & -13.97 \\
\hline Pintomyia christenseni & MT & Diamantino & -56.45 & -14.41 \\
\hline Pintomyia christenseni & MT & Alta Floresta & -56.09 & -9.88 \\
\hline Pintomyia christenseni & MT & Barra do Garças & -52.26 & -15.89 \\
\hline Pintomyia christenseni & MT & Brasnorte & -57.98 & -12.15 \\
\hline Pintomyia christenseni & MT & Chapada dos Guimarães & -55.75 & -15.46 \\
\hline Pintomyia christenseni & MT & Claúdia & -54.88 & -11.49 \\
\hline Pintomyia christenseni & MT & Gaúcha do Norte & -53.08 & -13.24 \\
\hline Pintomyia christenseni & MT & General Carneiro & -52.76 & -15.71 \\
\hline Pintomyia christenseni & MT & Jaciara & -54.95 & 15.92 \\
\hline Pintomyia christenseni & MT & Mercelândia & -54.60 & -11.11 \\
\hline Pintomyia christenseni & MT & Nobres & -56.33 & -14.73 \\
\hline Pintomyia christenseni & MT & Nossa S. do Livramento & -56.37 & -15.80 \\
\hline Pintomyia christenseni & MT & Nova Ubiratã & -55.26 & -13.01 \\
\hline Pintomyia christenseni & MT & Peixoto de Azevedo & -54.98 & -10.22 \\
\hline Pintomyia christenseni & MT & Sinop & -55.49 & -11.83 \\
\hline Pintomyia christenseni & MT & Sorriso & -55.71 & -12.55 \\
\hline Pintomyia christenseni & MT & União do Sul & -54.36 & -11.52 \\
\hline Psathyromyia shannoni "sensu lato" & MT & Diamantino & -56.45 & -14.41 \\
\hline Psathyromyia shannoni"sensu lato" & MT & Barra dos Bugres & -57.18 & -15.08 \\
\hline Psathyromyia shannoni "sensu lato" & MT & Rosário d' Oeste & -56.43 & -14.84 \\
\hline Psathyromyia shannoni "sensu lato" & MT & Barra do Garças & -52.26 & -15.89 \\
\hline Psathyromyia shannoni "sensu lato" & MT & Nova Mutum & -56.08 & -13.83 \\
\hline Psathyromyia shannoni "sensu lato" & MT & Alta Floresta & -56.09 & -9.88 \\
\hline Psathyromyia shannoni "sensu lato" & MT & Apiacás & -57.45 & -9.54 \\
\hline Psathyromyia shannoni "sensu lato" & MT & Arenápolis & -56.82 & -14.43 \\
\hline Psathyromyia shannoni "sensu lato" & MT & Barão do Melgaço & -55.97 & -16.19 \\
\hline Psathyromyia shannoni "sensu lato" & MT & Chapada dos Guimarães & -55.75 & -15.46 \\
\hline Psathyromyia shannoni "sensu lato" & MT & Cuiabá & -56.10 & -15.60 \\
\hline Psathyromyia shannoni "sensu lato" & MT & Gaúcha do Norte & -53.08 & -13.24 \\
\hline Psathyromyia shannoni "sensu lato" & MT & Jaciara & -54.95 & -15.92 \\
\hline
\end{tabular}




\begin{tabular}{|c|c|c|c|c|}
\hline Sandfly species & State code & Municipalities & Longitude & Latitude \\
\hline Psathyromyia shannoni "sensu lato" & MT & Juína & -58.74 & -11.38 \\
\hline Psathyromyia shannoni "sensu lato" & MT & Luciara & -50.57 & -10.75 \\
\hline Psathyromyia shannoni "sensu lato" & MT & Mercelândia & -54.60 & -11.11 \\
\hline Psathyromyia shannoni "sensu lato" & MT & Nobres & -56.33 & -14.73 \\
\hline Psathyromyia shannoni "sensu lato" & MT & Nossa S. do Livramento & -56.37 & -15.80 \\
\hline Psathyromyia shannoni "sensu lato" & MT & Nova Bandeirante & -57.86 & -9.95 \\
\hline Psathyromyia shannoni "sensu lato" & MT & Nova Brasilândia & -54.57 & -14.57 \\
\hline Psathyromyia shannoni "sensu lato" & MT & Nova Canaã do Norte & -55.95 & -10.55 \\
\hline Psathyromyia shannoni "sensu lato" & MT & Nova Guarita & -55.40 & -10.30 \\
\hline Psathyromyia shannoni "sensu lato" & MT & Paranaíta & -56.48 & -9.67 \\
\hline Psathyromyia shannoni "sensu lato" & MT & Paratinga & -54.05 & -14.43 \\
\hline Psathyromyia shannoni "sensu lato" & MT & Peixoto de Azevedo & -54.98 & -10.22 \\
\hline Psathyromyia shannoni "sensu lato" & MT & Poconé & -56.62 & -16.26 \\
\hline Psathyromyia shannoni "sensu lato" & MT & Porto Alegre do Norte & -51.63 & -10.87 \\
\hline Psathyromyia shannoni "sensu lato" & MT & Poxoréo & -54.38 & -15.83 \\
\hline Psathyromyia shannoni "sensu lato" & MT & Primavera do Leste & -54.30 & -15.56 \\
\hline Psathyromyia shannoni "sensu lato" & MT & Rondonópolis & -54.64 & -16.47 \\
\hline Psathyromyia shannoni "sensu lato" & MT & Rosário Oeste & -56.43 & -14.84 \\
\hline Psathyromyia shannoni "sensu lato" & MT & Santo Antônio do Leverger & -56.08 & -15.87 \\
\hline Psathyromyia shannoni "sensu lato" & MT & São José dos 4 Marcos & -58.17 & -15.17 \\
\hline Psathyromyia shannoni "sensu lato" & MT & Sinop & -55.49 & -11.83 \\
\hline Psathyromyia shannoni "sensu lato" & MT & Sorriso & -55.71 & -12.55 \\
\hline Psathyromyia shannoni "sensu lato" & MT & Várzea Grande & -56.13 & -15.65 \\
\hline Psathyromyia shannoni "sensu lato" & $\mathrm{GO}$ & Colinas do Sul & -48.08 & -14.15 \\
\hline Psathyromyia shannoni "sensu lato" & $\mathrm{GO}$ & Divinópolis & -46.39 & -13.29 \\
\hline Psathyromyia shannoni "sensu lato" & $\mathrm{GO}$ & Goiás & -50.13 & -15.93 \\
\hline Psathyromyia shannoni "sensu lato" & $\mathrm{GO}$ & Goiatuba & -49.35 & -18.01 \\
\hline Psathyromyia shannoni "sensu lato" & $\mathrm{GO}$ & Maurilândia & -50.33 & -17.97 \\
\hline Psathyromyia shannoni "sensu lato" & $\mathrm{GO}$ & Minaçu & -48.22 & -13.53 \\
\hline Psathyromyia shannoni "sensu lato" & $\mathrm{GO}$ & Quirinópolis & -50.50 & -18.53 \\
\hline Psathyromyia shannoni "sensu lato" & $\mathrm{GO}$ & Itumbiara & -49.22 & -18.42 \\
\hline Psathyromyia shannoni "sensu lato" & $\mathrm{GO}$ & Sítio d'Abadia & -46.25 & -14.80 \\
\hline Psathyromyia shannoni "sensu lato" & $\mathrm{GO}$ & Amaro Leite & -49.15 & -13.97 \\
\hline Psathyromyia shannoni "sensu lato" & $\mathrm{GO}$ & Itapaci & -49.57 & -14.95 \\
\hline Psathyromyia shannoni "sensu lato" & $\mathrm{GO}$ & Mineiros & -49.53 & -16.83 \\
\hline Psathyromyia shannoni "sensu lato" & GO & Corumbaíba & -48.56 & -18.14 \\
\hline Psathyromyia shannoni "sensu lato" & $\mathrm{GO}$ & Anápolis & -48.95 & -16.32 \\
\hline Psathyromyia shannoni "sensu lato" & $\mathrm{GO}$ & Maurilândia & -50.31 & -18.00 \\
\hline Psathyromyia shannoni "sensu lato" & DF & Brasília & -47.42 & -15.78 \\
\hline Psathyromyia shannoni "sensu lato" & MS & Alcinópolis & -52.87 & -20.44 \\
\hline Psathyromyia shannoni "sensu lato" & MS & Anastácio & -55.81 & -20.48 \\
\hline Psathyromyia shannoni "sensu lato" & MS & Antonio João & -55.95 & -22.19 \\
\hline Psathyromyia shannoni "sensu lato" & MS & Aquidauana & -55.79 & -20.47 \\
\hline Psathyromyia shannoni "sensu lato" & MS & Bela Vista & -56.52 & -22.11 \\
\hline Psathyromyia shannoni "sensu lato" & MS & Bodoquena & -56.72 & -20.54 \\
\hline Psathyromyia shannoni "sensu lato" & MS & Campo Grande & -54.65 & -20.44 \\
\hline Psathyromyia shannoni "sensu lato" & MS & Caarapó & -54.80 & -22.63 \\
\hline Psathyromyia shannoni "sensu lato" & MS & Camapuã & -54.04 & -19.53 \\
\hline Psathyromyia shannoni "sensu lato" & MS & Corguinho & -54.83 & -19.83 \\
\hline Psathyromyia shannoni "sensu lato" & MS & Corumbá & -57.65 & -19.01 \\
\hline Psathyromyia shannoni "sensu lato" & MS & Dourados & -54.81 & -22.22 \\
\hline Psathyromyia shannoni "sensu lato" & MS & Guia Lopes da Laguna & -56.11 & -21.46 \\
\hline Psathyromyia shannoni "sensu lato" & MS & Jardim & -56.14 & -21.48 \\
\hline
\end{tabular}




\begin{tabular}{|c|c|c|c|c|}
\hline Sandfly species & State code & Municipalities & Longitude & Latitude \\
\hline Psathyromyia shannoni "sensu lato" & MS & Nova Andradina & -53.34 & -22.23 \\
\hline Psathyromyia shannoni "sensu lato" & MS & Novo Horizonte do Sul & -53.80 & -22.61 \\
\hline Psathyromyia shannoni "sensu lato" & MS & Nioaque & -55.83 & -21.14 \\
\hline Psathyromyia shannoni "sensu lato" & MS & Bonito & -56.47 & -21.13 \\
\hline Psathyromyia shannoni "sensu lato" & MS & Maracajú & -55.17 & -21.61 \\
\hline Psathyromyia shannoni "sensu lato" & MS & Rio Verde de Mato Grosso & -54.84 & -18.92 \\
\hline Psathyromyia shannoni "sensu lato" & MS & Nhecolânia & -57.07 & -17.27 \\
\hline Psathyromyia runoides & MT & Peixoto de Azevedo & -54.98 & -10.22 \\
\hline Psathyromyia runoides & MT & Nova Mutum & -56.08 & -13.83 \\
\hline Psathyromyia runoides & MT & Alta Floresta & -56.09 & -9.88 \\
\hline Psathyromyia runoides & MT & Alto do Paraguai & -56.48 & -14.51 \\
\hline Psathyromyia runoides & MT & Apiacás & -57.45 & -9.54 \\
\hline Psathyromyia runoides & MT & Arenápolis & -56.82 & -14.43 \\
\hline Psathyromyia runoides & MT & Aripuanã & -60.63 & -9.17 \\
\hline Psathyromyia runoides & MT & Barra do Garças & -52.26 & -15.89 \\
\hline Psathyromyia runoides & MT & Brasnorte & -57.98 & -12.15 \\
\hline Psathyromyia runoides & MT & Campo Novo do Parecis & -57.88 & -13.67 \\
\hline Psathyromyia runoides & MT & Campo Verde & -55.16 & -15.54 \\
\hline Psathyromyia runoides & MT & Canabrava do Norte & -51.83 & -11.03 \\
\hline Psathyromyia runoides & MT & Canarana & -52.27 & -13.55 \\
\hline Psathyromyia runoides & MT & Carolina & -55.83 & -9.96 \\
\hline Psathyromyia runoides & MT & Chapada dos Guimarães & -55.75 & -15.46 \\
\hline Psathyromyia runoides & MT & Colniza & -59.03 & -9.40 \\
\hline Psathyromyia runoides & MT & Cuiabá & -56.10 & -15.60 \\
\hline Psathyromyia runoides & MT & Feliz Natal & -54.92 & -12.39 \\
\hline Psathyromyia runoides & MT & Gaúcha do Norte & -53.08 & -13.24 \\
\hline Psathyromyia runoides & MT & Guarantã do Norte & -54.90 & -9.78 \\
\hline Psathyromyia runoides & MT & Itiquira & -54.14 & -17.21 \\
\hline Psathyromyia runoides & MT & Lucas do Rio Verde & -55.91 & -13.05 \\
\hline Psathyromyia runoides & MT & Mercelândia & -54.60 & -11.11 \\
\hline Psathyromyia runoides & MT & Nobres & -56.33 & -14.73 \\
\hline Psathyromyia runoides & MT & Nossa S. do Livramento & -56.37 & -15.80 \\
\hline Psathyromyia runoides & MT & Nova Canaã do Norte & -55.95 & -10.55 \\
\hline Psathyromyia runoides & MT & Nova Santana Helena & -53.35 & -10.82 \\
\hline Psathyromyia runoides & MT & Nova Ubiratã & -55.26 & -13.01 \\
\hline Psathyromyia runoides & MT & Novo Horizonte do Norte & -57.35 & -11.41 \\
\hline Psathyromyia runoides & MT & Paranaíta & -56.48 & -9.67 \\
\hline Psathyromyia runoides & MT & Paratinga & -54.05 & -14.43 \\
\hline Psathyromyia runoides & MT & Poconé & -56.62 & -16.26 \\
\hline Psathyromyia runoides & MT & Porto Alegre do Norte & -51.63 & -10.87 \\
\hline Psathyromyia runoides & MT & Poxoréo & -54.38 & -15.83 \\
\hline Psathyromyia runoides & MT & Primavera do Leste & -54.30 & -15.56 \\
\hline Psathyromyia runoides & MT & Santo Antônio do Leverger & -56.08 & -15.87 \\
\hline Psathyromyia runoides & MT & Sapezal & -57.20 & -14.97 \\
\hline Psathyromyia runoides & MT & Serra da Nova Dourado & -51.58 & -11.97 \\
\hline Psathyromyia runoides & MT & Sinop & -55.49 & -11.83 \\
\hline Psathyromyia runoides & MT & Taboporã & -56.62 & -10.80 \\
\hline Psathyromyia runoides & MT & Tapurah & -56.50 & -12.53 \\
\hline Psathyromyia runoides & MT & União do Sul & -54.36 & -11.52 \\
\hline Psathyromyia runoides & MT & Várzea Grande & -56.13 & -15.65 \\
\hline Psathyromyia runoides & MT & Ipiranga do Norte & -56.05 & -15.35 \\
\hline Psathyromyia runoides & MS & Rio Verde de MT & -54.84 & -18.92 \\
\hline Psathyromyia runoides & MS & Coxim & -54.76 & -18.51 \\
\hline
\end{tabular}




\begin{tabular}{|c|c|c|c|c|}
\hline Sandfly species & State code & Municipalities & Longitude & Latitude \\
\hline Psathyromyia lutziana & MT & Barra do Garças & -52.26 & -15.89 \\
\hline Psathyromyia lutziana & MT & Varzea Grande & -56.13 & -15.65 \\
\hline Psathyromyia lutziana & MT & Cuiabá & -56.10 & -15.60 \\
\hline Psathyromyia lutziana & MT & Alta Floresta & -56.09 & -9.88 \\
\hline Psathyromyia lutziana & MT & Alto Araguaia & -53.22 & -17.31 \\
\hline Psathyromyia lutziana & MT & Alto do Paraguai & -56.48 & -14.51 \\
\hline Psathyromyia lutziana & MT & Apiacás & -57.45 & -9.54 \\
\hline Psathyromyia lutziana & MT & Araguainha & -53.08 & -16.82 \\
\hline Psathyromyia lutziana & MT & Canabrava do Norte & -51.83 & -11.03 \\
\hline Psathyromyia lutziana & MT & Chapada dos Guimarães & -55.75 & -15.46 \\
\hline Psathyromyia lutziana & MT & Cládia & -54.88 & -11.49 \\
\hline Psathyromyia lutziana & MT & Confresa & -51.56 & -10.64 \\
\hline Psathyromyia lutziana & MT & Feliz Natal & -54.92 & -12.39 \\
\hline Psathyromyia lutziana & MT & Itiquira & -54.14 & -17.21 \\
\hline Psathyromyia lutziana & MT & Jaciara & -54.95 & -15.92 \\
\hline Psathyromyia lutziana & MT & Juína & -58.74 & -11.38 \\
\hline Psathyromyia lutziana & MT & Juscimeira & -54.88 & -16.05 \\
\hline Psathyromyia lutziana & MT & Lucas do Rio Verde & -10.75 & -50.57 \\
\hline Psathyromyia lutziana & MT & Mercelândia & -54.60 & -11.11 \\
\hline Psathyromyia lutziana & MT & Matupá & -54.90 & -10.05 \\
\hline Psathyromyia lutziana & MT & Nossa S. do Livramento & -56.37 & -15.80 \\
\hline Psathyromyia lutziana & MT & Nova Marilândia & -56.57 & -14.21 \\
\hline Psathyromyia lutziana & MT & Nova Maringá & -57.06 & -13.01 \\
\hline Psathyromyia lutziana & MT & Nova Mutum & -56.08 & -13.83 \\
\hline Psathyromyia lutziana & MT & Nova Ubiratã & -55.26 & -13.01 \\
\hline Psathyromyia lutziana & MT & Novo Horizonte do Norte & -57.35 & -11.41 \\
\hline Psathyromyia lutziana & MT & Novo São Joaquim & -53.02 & -14.90 \\
\hline Psathyromyia lutziana & MT & Paranaíta & -56.48 & -9.67 \\
\hline Psathyromyia lutziana & MT & Paratinga & -54.05 & -14.43 \\
\hline Psathyromyia lutziana & MT & Porto Alegre do Norte & -51.63 & -10.87 \\
\hline Psathyromyia lutziana & MT & Porto dos Gaúchos & -57.41 & -11.52 \\
\hline Psathyromyia lutziana & MT & Poxoréo & -54.38 & -15.83 \\
\hline Psathyromyia lutziana & MT & Ribeirão Cascalheira & -51.82 & -12.92 \\
\hline Psathyromyia lutziana & MT & Rondonópolis & -54.64 & -16.47 \\
\hline Psathyromyia lutziana & MT & Rosário D’ Oeste & -56.43 & -14.84 \\
\hline Psathyromyia lutziana & MT & Santo Antônio do Leverger & -56.08 & -15.87 \\
\hline Psathyromyia lutziana & MT & São Felix do Araguaia & -50.67 & -11.61 \\
\hline Psathyromyia lutziana & MT & São Pedro da Cipa & -54.91 & -15.99 \\
\hline Psathyromyia lutziana & MT & Serra da Nova Dourado & -51.58 & -11.97 \\
\hline Psathyromyia lutziana & MT & Sinop & -55.49 & -11.83 \\
\hline Psathyromyia lutziana & MT & Taboporã & -56.62 & -10.80 \\
\hline Psathyromyia lutziana & MT & Tangará da Serra & -57.50 & -14.62 \\
\hline Psathyromyia lutziana & MT & União do Sul & -54.36 & -11.52 \\
\hline Psathyromyia lutziana & MT & Vila Rica & -51.11 & -10.01 \\
\hline Psathyromyia lutziana & MT & Itanhangá & -56.64 & -12.23 \\
\hline Psathyromyia lutziana & $\mathrm{GO}$ & Itumbiara & -49.22 & -18.42 \\
\hline Psathyromyia lutziana & $\mathrm{DF}$ & Brasília & -47.42 & -15.78 \\
\hline Psathyromyia lutziana & MS & Corguinho & -54.83 & -19.83 \\
\hline Psathyromyia lutziana & MS & Rochedo & -54.89 & -19.95 \\
\hline Psathyromyia lutziana & MS & Nioaque & -55.83 & -21.14 \\
\hline Psathyromyia lutziana & MS & Terenos & -54.86 & -20.44 \\
\hline Psathyromyia lutziana & MS & Três Lagoas & -51.68 & -20.75 \\
\hline Psathyromyia lutziana & MS & Camapuã & -54.04 & -19.53 \\
\hline
\end{tabular}




\begin{tabular}{|c|c|c|c|c|}
\hline Sandfly species & State code & Municipalities & Longitude & Latitude \\
\hline Psathyromyia aragaoi & MT & Peixoto de Azevedo & -54.98 & -10.22 \\
\hline Psathyromyia aragaoi & MT & Varzea Grande & -56.13 & -15.65 \\
\hline Psathyromyia aragaoi & MT & Nova Mutum & -56.08 & -13.83 \\
\hline Psathyromyia aragaoi & MT & Cuiabá & -56.10 & -15.60 \\
\hline Psathyromyia aragaoi & MT & Água Boa & -52.16 & -14.05 \\
\hline Psathyromyia aragaoi & MT & Alta Floresta & -56.09 & -9.88 \\
\hline Psathyromyia aragaoi & MT & Alto Araguaia & -53.22 & -17.31 \\
\hline Psathyromyia aragaoi & MT & Alto do Paraguai & -56.48 & -14.51 \\
\hline Psathyromyia aragaoi & MT & Apiacás & -57.45 & -9.54 \\
\hline Psathyromyia aragaoi & MT & Barão do Melgaço & -55.97 & -16.19 \\
\hline Psathyromyia aragaoi & MT & Barra do Garças & -52.26 & -15.89 \\
\hline Psathyromyia aragaoi & MT & Brasnorte & -57.98 & -12.15 \\
\hline Psathyromyia aragaoi & MT & Cáceres & -57.68 & -16.07 \\
\hline Psathyromyia aragaoi & MT & Campinápolis & -52.88 & -14.50 \\
\hline Psathyromyia aragaoi & MT & Campo Novo do Parecis & -57.88 & -13.67 \\
\hline Psathyromyia aragaoi & MT & Campo Verde & -55.16 & -15.54 \\
\hline Psathyromyia aragaoi & MT & Canabrava do Norte & -51.83 & -11.03 \\
\hline Psathyromyia aragaoi & MT & Carolina & -55.83 & -9.96 \\
\hline Psathyromyia aragaoi & MT & Chapada dos Guimarães & -55.75 & -15.46 \\
\hline Psathyromyia aragaoi & MT & Cláudia & -54.88 & -11.49 \\
\hline Psathyromyia aragaoi & MT & Colíder & -55.45 & -10.81 \\
\hline Psathyromyia aragaoi & MT & Comodoro & -59.79 & -13.66 \\
\hline Psathyromyia aragaoi & MT & Diamantino & -56.45 & -14.41 \\
\hline Psathyromyia aragaoi & MT & Dom Aquino & -54.55 & -15.48 \\
\hline Psathyromyia aragaoi & MT & Feliz Natal & -54.92 & -12.39 \\
\hline Psathyromyia aragaoi & MT & Gerneral Carneiro & -52.76 & -15.71 \\
\hline Psathyromyia aragaoi & MT & Guarantã do Norte & -54.90 & -9.78 \\
\hline Psathyromyia aragaoi & MT & Itiquira & -54.14 & -17.21 \\
\hline Psathyromyia aragaoi & MT & Mercelândia & -54.60 & -11.11 \\
\hline Psathyromyia aragaoi & MT & Nobres & -56.33 & -14.73 \\
\hline Psathyromyia aragaoi & MT & Nossa S. do Livramento & -56.37 & -15.80 \\
\hline Psathyromyia aragaoi & MT & Nova Brasilândia & -54.57 & -14.57 \\
\hline Psathyromyia aragaoi & MT & Nova Maringá & -57.06 & -13.01 \\
\hline Psathyromyia aragaoi & MT & Nova Ubiratã & -55.26 & -13.01 \\
\hline Psathyromyia aragaoi & MT & Novo Horizonte do Norte & -57.35 & -11.41 \\
\hline Psathyromyia aragaoi & MT & Novo São Joaquim & -53.02 & -14.90 \\
\hline Psathyromyia aragaoi & MT & Paranaíta & -56.48 & -9.67 \\
\hline Psathyromyia aragaoi & MT & Paratinga & -54.05 & -14.43 \\
\hline Psathyromyia aragaoi & MT & Planalto da Serra & -55.27 & -10.44 \\
\hline Psathyromyia aragaoi & MT & Poconé & -56.62 & -16.26 \\
\hline Psathyromyia aragaoi & MT & Pontes de Lacerda & -59.37 & -15.20 \\
\hline Psathyromyia aragaoi & MT & Porto Esperidião & -58.47 & -15.85 \\
\hline Psathyromyia aragaoi & MT & Poxoréo & -54.38 & -15.83 \\
\hline Psathyromyia aragaoi & MT & Primavera do Leste & -54.30 & -15.56 \\
\hline Psathyromyia aragaoi & MT & Rondonópolis & -54.64 & -16.47 \\
\hline Psathyromyia aragaoi & MT & Rosário Oeste & -56.43 & -14.84 \\
\hline Psathyromyia aragaoi & MT & Santa Rita do Trivelato & -55.26 & -13.81 \\
\hline Psathyromyia aragaoi & MT & Santo Antônio do Leste & -53.62 & -14.83 \\
\hline Psathyromyia aragaoi & MT & Santo Antônio do Leverger & -56.08 & -15.87 \\
\hline Psathyromyia aragaoi & MT & São Felix do Araguaia & -50.67 & -11.61 \\
\hline Psathyromyia aragaoi & MT & Sinop & -55.49 & -11.83 \\
\hline Psathyromyia aragaoi & MT & Sorriso & -55.71 & -12.55 \\
\hline Psathyromyia aragaoi & MT & Taboporã & -56.62 & -10.80 \\
\hline
\end{tabular}




\begin{tabular}{|c|c|c|c|c|}
\hline Sandfly species & State code & Municipalities & Longitude & Latitude \\
\hline Psathyromyia aragaoi & MT & Tapurah & -56.50 & -12.53 \\
\hline Psathyromyia aragaoi & MT & Terra nova do Norte & -55.23 & -10.51 \\
\hline Psathyromyia aragaoi & MT & União do Sul & -54.36 & -11.52 \\
\hline Psathyromyia aragaoi & $\mathrm{GO}$ & Itumbiara & -49.22 & -18.42 \\
\hline Psathyromyia aragaoi & DF & Brasília & -47.42 & -15.78 \\
\hline Psathyromyia aragaoi & MS & Alcinópolis & -53.70 & -18.31 \\
\hline Psathyromyia aragaoi & MS & Bela Vista & -56.52 & -22.11 \\
\hline Psathyromyia aragaoi & MS & Caarapó (Aldeia Jacaré) & -54.80 & -22.63 \\
\hline Psathyromyia aragaoi & MS & Campo Grande & -54.65 & -20.44 \\
\hline Psathyromyia aragaoi & MS & Camapuã & -54.04 & -19.53 \\
\hline Psathyromyia aragaoi & MS & Corumbá & -57.65 & -19.01 \\
\hline Psathyromyia aragaoi & MS & Corguinho & -54.83 & -19.83 \\
\hline Psathyromyia aragaoi & MS & Coxim & -54.76 & -18.51 \\
\hline Psathyromyia aragaoi & MS & Dourados & -54.81 & -22.22 \\
\hline Psathyromyia aragaoi & MS & Nioaque & -55.83 & -21.14 \\
\hline Psathyromyia aragaoi & MS & Naviraí & -54.22 & -23.13 \\
\hline Psathyromyia aragaoi & MS & Novo Horizonte do Sul & -53.80 & -22.61 \\
\hline Psathyromyia aragaoi & MS & Nioaque & -55.83 & -21.14 \\
\hline Psathyromyia aragaoi & MS & Bonito & -56.47 & -21.13 \\
\hline Psathyromyia aragaoi & MS & Paranhos & -55.43 & -23.89 \\
\hline Psathyromyia aragaoi & MS & São Gabriel d' Oeste & -54.57 & -19.40 \\
\hline Psathyromyia aragaoi & MS & Rio Verde de Mato Grosso & -54.84 & -18.92 \\
\hline Psathyromyia aragaoi & MS & Nhecolânia & -57.07 & -17.27 \\
\hline Psathyromyia punctigeniculata & MT & Barra do Garças & -52.26 & -15.89 \\
\hline Psathyromyia punctigeniculata & MT & Varzea Grande & -56.13 & -15.65 \\
\hline Psathyromyia punctigeniculata & MT & Nova Mutum & -56.08 & -13.83 \\
\hline Psathyromyia punctigeniculata & MT & Água Boa & -52.16 & -14.05 \\
\hline Psathyromyia punctigeniculata & MT & Alto Araguaia & -53.22 & -17.31 \\
\hline Psathyromyia punctigeniculata & MT & Brasnorte & -57.98 & -12.15 \\
\hline Psathyromyia punctigeniculata & MT & Campinápolis & -52.88 & -14.50 \\
\hline Psathyromyia punctigeniculata & MT & Canabrava do Norte & -51.83 & -11.03 \\
\hline Psathyromyia punctigeniculata & MT & Chapada dos Guimarães & -55.75 & -15.46 \\
\hline Psathyromyia punctigeniculata & MT & Claúdia & -54.88 & -11.49 \\
\hline Psathyromyia punctigeniculata & MT & Colniza & -59.03 & -9.40 \\
\hline Psathyromyia punctigeniculata & MT & Confresa & -51.56 & -10.64 \\
\hline Psathyromyia punctigeniculata & MT & Cuiabá & -56.10 & -15.60 \\
\hline Psathyromyia punctigeniculata & MT & Diamantino & -56.45 & -14.41 \\
\hline Psathyromyia punctigeniculata & MT & Gaúcha do Norte & -53.08 & -13.24 \\
\hline Psathyromyia punctigeniculata & MT & Gerneral Carneiro & -52.76 & -15.71 \\
\hline Psathyromyia punctigeniculata & MT & Guarantã do Norte & -54.90 & -9.78 \\
\hline Psathyromyia punctigeniculata & MT & Jaciara & -54.95 & -15.92 \\
\hline Psathyromyia punctigeniculata & MT & Lucas do Rio Verde & -55.91 & -13.05 \\
\hline Psathyromyia punctigeniculata & MT & Luciara & -50.57 & -10.75 \\
\hline Psathyromyia punctigeniculata & MT & Mercelândia & -54.60 & -11.11 \\
\hline Psathyromyia punctigeniculata & MT & Matupá & -54.90 & -10.05 \\
\hline Psathyromyia punctigeniculata & MT & Mirassol D' Oeste & -58.05 & -15.40 \\
\hline Psathyromyia punctigeniculata & MT & Nobres & -56.33 & -14.73 \\
\hline Psathyromyia punctigeniculata & MT & Nossa S. do Livramento & -56.37 & -15.80 \\
\hline Psathyromyia punctigeniculata & MT & Nova Brasilândia & -54.57 & -14.57 \\
\hline Psathyromyia punctigeniculata & MT & Nova Santana Helena & -53.35 & -10.82 \\
\hline Psathyromyia punctigeniculata & MT & Nova Ubiratã & -55.26 & -13.01 \\
\hline Psathyromyia punctigeniculata & MT & Novo São Joaquim & -53.02 & -14.90 \\
\hline Psathyromyia punctigeniculata & MT & Paratinga & -54.05 & -14.43 \\
\hline
\end{tabular}




\begin{tabular}{|c|c|c|c|c|}
\hline Sandfly species & State code & Municipalities & Longitude & Latitude \\
\hline Psathyromyia punctigeniculata & MT & Peixoto de Azevedo & -54.98 & -10.22 \\
\hline Psathyromyia punctigeniculata & MT & Poconé & -56.62 & -16.26 \\
\hline Psathyromyia punctigeniculata & MT & Ponte Branca & -56.78 & -16.08 \\
\hline Psathyromyia punctigeniculata & MT & Porto Alegre do Norte & -51.63 & -10.87 \\
\hline Psathyromyia punctigeniculata & MT & Poxoréo & -54.38 & -15.83 \\
\hline Psathyromyia punctigeniculata & MT & Ribeirão Cascalheira & -51.82 & -12.92 \\
\hline Psathyromyia punctigeniculata & MT & Rosário D’ Oeste & -56.43 & -14.84 \\
\hline Psathyromyia punctigeniculata & MT & Santa Rita do Trivelato & -55.26 & -13.81 \\
\hline Psathyromyia punctigeniculata & MT & Santo Antônio do Leverger & -56.08 & -15.87 \\
\hline Psathyromyia punctigeniculata & MT & São Felix do Araguaia & -50.67 & -11.61 \\
\hline Psathyromyia punctigeniculata & MT & Sinop & -55.49 & -11.83 \\
\hline Psathyromyia punctigeniculata & MT & Sorriso & -55.71 & -12.55 \\
\hline Psathyromyia punctigeniculata & MT & União do Sul & -54.36 & -11.52 \\
\hline Psathyromyia punctigeniculata & MT & Vila Rica & -51.11 & -10.01 \\
\hline Psathyromyia punctigeniculata & MT & Ipiranga do Norte & -56.05 & -15.35 \\
\hline Psathyromyia punctigeniculata & $\mathrm{GO}$ & Itumbiara & -49.22 & -18.42 \\
\hline Psathyromyia punctigeniculata & $\mathrm{GO}$ & Israelândia & -50.90 & -16.35 \\
\hline Psathyromyia punctigeniculata & $\mathrm{GO}$ & Amaro Leite & -49.15 & -13.97 \\
\hline Psathyromyia punctigeniculata & MS & Bela Vista & -56.52 & -22.11 \\
\hline Psathyromyia punctigeniculata & MS & Campo Grande & -54.65 & -20.44 \\
\hline Psathyromyia punctigeniculata & MS & Camapuã & -54.04 & -19.53 \\
\hline Psathyromyia punctigeniculata & MS & Corguinho & -54.83 & -19.83 \\
\hline Psathyromyia punctigeniculata & MS & Rio Negro & -54.99 & -19.45 \\
\hline Psathyromyia punctigeniculata & MS & Terenos & -54.86 & -20.44 \\
\hline Psathyromyia punctigeniculata & MS & Jardim & -56.14 & -21.48 \\
\hline Psathyromyia punctigeniculata & MS & Novo Horizonte do Sul & -53.80 & -22.61 \\
\hline Psathyromyia punctigeniculata & MS & Nova Andradina & -53.34 & -22.23 \\
\hline Psathyromyia punctigeniculata & MS & Bonito & -56.47 & -21.13 \\
\hline Psathyromyia punctigeniculata & MS & Maracajú & -55.17 & -21.61 \\
\hline Psathyromyia punctigeniculata & MS & Rio Verde de Mato Grosso & -54.84 & -18.92 \\
\hline Psathyromyia punctigeniculata & MS & Três Lagoas & -51.68 & -20.75 \\
\hline Psathyromyia hermanlenti & MT & Peixoto de Azevedo & -54.98 & -10.22 \\
\hline Psathyromyia hermanlenti & MT & Varzea Grande & -56.13 & -15.65 \\
\hline Psathyromyia hermanlenti & MT & Nova Mutum & -56.08 & -13.83 \\
\hline Psathyromyia hermanlenti & MT & Barra do Garças & -52.26 & -15.89 \\
\hline Psathyromyia hermanlenti & MT & Água Boa & -52.16 & -14.05 \\
\hline Psathyromyia hermanlenti & MT & Alto da Boa Vista & -51.38 & -11.66 \\
\hline Psathyromyia hermanlenti & MT & Alto do Paraguai & -56.48 & -14.51 \\
\hline Psathyromyia hermanlenti & MT & Araguaína & -51.83 & -15.73 \\
\hline Psathyromyia hermanlenti & MT & Arenápolis & -56.82 & -14.43 \\
\hline Psathyromyia hermanlenti & MT & Aripuanã & -60.63 & -9.17 \\
\hline Psathyromyia hermanlenti & MT & Barão do Melgaço & -55.97 & -16.19 \\
\hline Psathyromyia hermanlenti & MT & Barra dos Bugres & -57.18 & -15.08 \\
\hline Psathyromyia hermanlenti & MT & Cáceres & -57.68 & -16.07 \\
\hline Psathyromyia hermanlenti & MT & Carolina & -55.83 & -9.96 \\
\hline Psathyromyia hermanlenti & MT & Chapada dos Guimarães & -55.75 & -15.46 \\
\hline Psathyromyia hermanlenti & MT & Claúdia & -54.88 & -11.49 \\
\hline Psathyromyia hermanlenti & MT & Colíder & -55.45 & -10.81 \\
\hline Psathyromyia hermanlenti & MT & Confresa & -51.56 & -10.64 \\
\hline Psathyromyia hermanlenti & MT & Cuiabá & -56.10 & -15.60 \\
\hline Psathyromyia hermanlenti & MT & Diamantino & -56.45 & -14.41 \\
\hline Psathyromyia hermanlenti & MT & Feliz Natal & -54.92 & -12.39 \\
\hline Psathyromyia hermanlenti & MT & Gaúcha do Norte & -53.08 & -13.24 \\
\hline
\end{tabular}




\begin{tabular}{|c|c|c|c|c|}
\hline Sandfly species & State code & Municipalities & Longitude & Latitude \\
\hline Psathyromyia hermanlenti & MT & Gerneral Carneiro & -52.76 & -15.71 \\
\hline Psathyromyia hermanlenti & MT & Guarantã do Norte & -54.90 & -9.78 \\
\hline Psathyromyia hermanlenti & MT & Itiquira & -54.14 & -17.21 \\
\hline Psathyromyia hermanlenti & MT & Jaciara & -54.95 & -15.92 \\
\hline Psathyromyia hermanlenti & MT & Juara & -57.52 & -11.55 \\
\hline Psathyromyia hermanlenti & MT & Juína & -58.74 & -11.38 \\
\hline Psathyromyia hermanlenti & MT & Lucas do Rio Verde & -55.91 & -13.05 \\
\hline Psathyromyia hermanlenti & MT & Mercelândia & -54.60 & -11.11 \\
\hline Psathyromyia hermanlenti & MT & Matupá & -54.90 & -10.05 \\
\hline Psathyromyia hermanlenti & MT & Mirassol d' Oeste & -58.05 & -15.40 \\
\hline Psathyromyia hermanlenti & MT & Nobres & -56.33 & -14.73 \\
\hline Psathyromyia hermanlenti & MT & Notelândia & -56.80 & -14.42 \\
\hline Psathyromyia hermanlenti & MT & Nossa S. do Livramento & -56.37 & -15.80 \\
\hline Psathyromyia hermanlenti & MT & Nova Bandeirantes & -57.86 & -9.95 \\
\hline Psathyromyia hermanlenti & MT & Nova Canaã do Norte & -55.95 & -10.55 \\
\hline Psathyromyia hermanlenti & MT & Nova Guarita & -55.40 & -10.30 \\
\hline Psathyromyia hermanlenti & MT & Nova Maringá & -57.06 & -13.01 \\
\hline Psathyromyia hermanlenti & MT & Nova Mutum & -56.08 & -13.83 \\
\hline Psathyromyia hermanlenti & MT & Nova Santana Helena & -55.26 & -13.01 \\
\hline Psathyromyia hermanlenti & MT & Nova Ubiratã & -55.26 & -13.01 \\
\hline Psathyromyia hermanlenti & MT & Novo Horizonte do Norte & -57.35 & -11.41 \\
\hline Psathyromyia hermanlenti & MT & Novo São Joaquim & -53.02 & -14.90 \\
\hline Psathyromyia hermanlenti & MT & Paranaíta & -56.48 & -9.67 \\
\hline Psathyromyia hermanlenti & MT & Paratinga & -54.05 & -14.43 \\
\hline Psathyromyia hermanlenti & MT & Pedra Preta & -54.47 & -16.62 \\
\hline Psathyromyia hermanlenti & MT & Peixoto de Azevedo & -54.98 & -10.22 \\
\hline Psathyromyia hermanlenti & MT & Planalto da Serra & -55.27 & -10.44 \\
\hline Psathyromyia hermanlenti & MT & Poconé & -56.62 & -16.26 \\
\hline Psathyromyia hermanlenti & MT & Pontal do Araguaia & -52.01 & -15.85 \\
\hline Psathyromyia hermanlenti & MT & Ponte Branca & -56.78 & -16.08 \\
\hline Psathyromyia hermanlenti & MT & Porto Alegre do Norte & -51.63 & -10.87 \\
\hline Psathyromyia hermanlenti & MT & Porto dos Gaúchos & -57.41 & -11.52 \\
\hline Psathyromyia hermanlenti & MT & Porto Esperidião & -58.47 & -15.85 \\
\hline Psathyromyia hermanlenti & MT & Poxoréo & -54.38 & -15.83 \\
\hline Psathyromyia hermanlenti & MT & Primavera do Leste & -54.30 & -15.56 \\
\hline Psathyromyia hermanlenti & MT & Ribeirãozinho & -52.67 & -16.45 \\
\hline Psathyromyia hermanlenti & MT & Rondonópolis & -54.64 & -16.47 \\
\hline Psathyromyia hermanlenti & MT & Rosário Oeste & -56.43 & -14.84 \\
\hline Psathyromyia hermanlenti & MT & Santo Antônio do Leverger & -56.08 & -15.87 \\
\hline Psathyromyia hermanlenti & MT & São Felix do Araguaia & -50.67 & -11.61 \\
\hline Psathyromyia hermanlenti & MT & São José dos 4 Marcos & -58.17 & -15.17 \\
\hline Psathyromyia hermanlenti & MT & Serra da Nova Dourado & -51.58 & -11.97 \\
\hline Psathyromyia hermanlenti & MT & Sinop & -55.49 & -11.83 \\
\hline Psathyromyia hermanlenti & MT & Sorriso & -55.71 & -12.55 \\
\hline Psathyromyia hermanlenti & MT & Taboporã & -56.62 & -10.80 \\
\hline Psathyromyia hermanlenti & MT & Tapurah & -56.50 & -12.53 \\
\hline Psathyromyia hermanlenti & MT & Terra nova do Norte & -55.23 & -10.51 \\
\hline Psathyromyia hermanlenti & MT & União do Sul & -54.36 & -11.52 \\
\hline Psathyromyia hermanlenti & MT & Itanhangá & -56.64 & -12.23 \\
\hline Psathyromyia hermanlenti & $\mathrm{GO}$ & Itumbiara & -49.22 & -18.42 \\
\hline Psathyromyia hermanlenti & MS & Água Clara & -52.87 & -20.44 \\
\hline Psathyromyia hermanlenti & MS & Alcinópolis & -53.70 & -18.31 \\
\hline Psathyromyia hermanlenti & MS & Anastácio & -55.81 & -20.48 \\
\hline
\end{tabular}




\begin{tabular}{|c|c|c|c|c|}
\hline Sandfly species & State code & Municipalities & Longitude & Latitude \\
\hline Psathyromyia hermanlenti & MS & Camapuã & -54.04 & -19.53 \\
\hline Psathyromyia hermanlenti & MS & Campo Grande & -54.65 & -20.44 \\
\hline Psathyromyia hermanlenti & MS & Corguinho & -54.83 & -19.83 \\
\hline Psathyromyia hermanlenti & MS & Coxim & -54.76 & -18.507 \\
\hline Psathyromyia hermanlenti & MS & Figueirão & -53.38 & -18.4 \\
\hline Psathyromyia hermanlenti & MS & Terenos & -54.86 & -20.44 \\
\hline Psathyromyia hermanlenti & MS & Costa Rica & -53.13 & -18.54 \\
\hline Psathyromyia hermanlenti & MS & Novo Horizonte do Sul & -53.80 & -22.61 \\
\hline Psathyromyia hermanlenti & MS & Pedro Gomes & -54.55 & -18.10 \\
\hline Psathyromyia hermanlenti & MS & Rio Verde de Mato Grosso & -54.84 & -18.92 \\
\hline Psychodopygus complexus & MT & Peixoto de Azevedo & -54.98 & -10.22 \\
\hline Psychodopygus complexus & MT & Nova Mutum & -56.08 & -13.83 \\
\hline Psychodopygus complexus & MT & Alta Floresta & -56.09 & -9.88 \\
\hline Psychodopygus complexus & MT & Campo Verde & -55.16 & -15.54 \\
\hline Psychodopygus complexus & MT & Chapada dos Guimarães & -55.75 & -15.46 \\
\hline Psychodopygus complexus & MT & Cládia & -54.88 & -11.49 \\
\hline Psychodopygus complexus & MT & Diamantino & -56.45 & -14.41 \\
\hline Psychodopygus complexus & MT & Dom Aquino & -54.55 & -15.48 \\
\hline Psychodopygus complexus & MT & Feliz Natal & -54.92 & -12.39 \\
\hline Psychodopygus complexus & MT & Gerneral Carneiro & -52.76 & -15.71 \\
\hline Psychodopygus complexus & MT & Guarantã do Norte & -54.90 & -9.78 \\
\hline Psychodopygus complexus & MT & Lucas do Rio Verde & -55.91 & -13.05 \\
\hline Psychodopygus complexus & MT & Mercelândia & -54.60 & -11.11 \\
\hline Psychodopygus complexus & MT & Matupá & -54.90 & -10.05 \\
\hline Psychodopygus complexus & MT & Nova Marilândia & -56.57 & -14.21 \\
\hline Psychodopygus complexus & MT & Nova Ubiratã & -55.26 & -13.01 \\
\hline Psychodopygus complexus & MT & Paranaíta & -56.48 & -9.67 \\
\hline Psychodopygus complexus & MT & Paratinga & -54.05 & -14.43 \\
\hline Psychodopygus complexus & MT & Porto dos Gaúchos & -57.41 & -11.52 \\
\hline Psychodopygus complexus & MT & Poxoréo & -54.38 & -15.83 \\
\hline Psychodopygus complexus & MT & Rondonópolis & -54.64 & -16.47 \\
\hline Psychodopygus complexus & MT & São José do Povo & -54.25 & -16.46 \\
\hline Psychodopygus complexus & MT & Serra da Nova Dourado & -51.58 & -11.97 \\
\hline Psychodopygus complexus & MT & Sinop & -55.49 & -11.83 \\
\hline Psychodopygus complexus & MT & Sorriso & -55.71 & -12.55 \\
\hline Psychodopygus complexus & MT & Tapurah & -56.50 & -12.53 \\
\hline Psychodopygus complexus & MT & União do Sul & -54.36 & -11.52 \\
\hline Psychodopygus davisi & MT & Nova Mutum & -56.08 & -13.83 \\
\hline Psychodopygus davisi & MT & Cuiabá & -56.10 & -15.60 \\
\hline Psychodopygus davisi & MT & Barra do Garças & -52.26 & -15.89 \\
\hline Psychodopygus davisi & MT & Alta Floresta & -56.09 & -9.88 \\
\hline Psychodopygus davisi & MT & Alto do Paraguai & -56.48 & -14.51 \\
\hline Psychodopygus davisi & MT & Apiacás & -57.45 & -9.54 \\
\hline Psychodopygus davisi & MT & Aripuanã & -60.63 & -9.17 \\
\hline Psychodopygus davisi & MT & Cáceres & -57.68 & -16.07 \\
\hline Psychodopygus davisi & MT & Campo Novo do Parecis & -57.88 & -13.67 \\
\hline Psychodopygus davisi & MT & Campo Verde & -55.16 & -15.54 \\
\hline Psychodopygus davisi & MT & Canabrava do Norte & -51.83 & -11.03 \\
\hline Psychodopygus davisi & MT & Chapada dos Guimarães & -55.75 & -15.46 \\
\hline Psychodopygus davisi & MT & Cláudia & -54.88 & -11.49 \\
\hline Psychodopygus davisi & MT & Colniza & -59.03 & -9.40 \\
\hline Psychodopygus davisi & MT & Comodoro & -59.79 & -13.66 \\
\hline Psychodopygus davisi & MT & Cotriguaçu & -58.41 & -9.85 \\
\hline
\end{tabular}




\begin{tabular}{|c|c|c|c|c|}
\hline Sandfly species & State code & Municipalities & Longitude & Latitude \\
\hline Psychodopygus davisi & MT & Diamantino & -56.45 & -14.41 \\
\hline Psychodopygus davisi & MT & Feliz Natal & -54.92 & -12.39 \\
\hline Psychodopygus davisi & MT & Gerneral Carneiro & -52.76 & -15.71 \\
\hline Psychodopygus davisi & MT & Guarantã do Norte & -54.90 & -9.78 \\
\hline Psychodopygus davisi & MT & Jaciara & -54.95 & -15.92 \\
\hline Psychodopygus davisi & MT & Jangada & -56.49 & -15.24 \\
\hline Psychodopygus davisi & MT & Juara & -57.52 & -11.55 \\
\hline Psychodopygus davisi & MT & Juína & -58.74 & -11.38 \\
\hline Psychodopygus davisi & MT & Juscimeira & -54.88 & -16.05 \\
\hline Psychodopygus davisi & MT & Lucas do Rio Verde & -55.91 & -13.05 \\
\hline Psychodopygus davisi & MT & Mercelândia & -54.60 & -11.11 \\
\hline Psychodopygus davisi & MT & Matupá & -54.90 & -10.05 \\
\hline Psychodopygus davisi & MT & Nobres & -56.33 & -14.73 \\
\hline Psychodopygus davisi & MT & Notelândia & -56.80 & -14.42 \\
\hline Psychodopygus davisi & MT & Nova Brasilândia & -54.57 & -14.57 \\
\hline Psychodopygus davisi & MT & Nova Lacerda & -59.35 & -14.28 \\
\hline Psychodopygus davisi & MT & Nova Marilândia & -56.57 & -14.21 \\
\hline Psychodopygus davisi & MT & Nova Maringá & -57.06 & -13.01 \\
\hline Psychodopygus davisi & MT & Nova Ubiratã & -55.26 & -13.01 \\
\hline Psychodopygus davisi & MT & Novo Horizonte do Norte & -57.35 & -11.41 \\
\hline Psychodopygus davisi & MT & Paranaíta & -56.48 & -9.67 \\
\hline Psychodopygus davisi & MT & Paratinga & -54.05 & -14.43 \\
\hline Psychodopygus davisi & MT & Peixoto de Azevedo & -54.98 & -10.22 \\
\hline Psychodopygus davisi & MT & Pontes de Lacerda & -59.37 & -15.20 \\
\hline Psychodopygus davisi & MT & Porto dos Gaúchos & -57.41 & -11.52 \\
\hline Psychodopygus davisi & MT & Porto Esperidião & -58.47 & -15.85 \\
\hline Psychodopygus davisi & MT & Poxoréo & -54.38 & -15.83 \\
\hline Psychodopygus davisi & MT & Rondonópolis & -54.64 & -16.47 \\
\hline Psychodopygus davisi & MT & Rosário Oeste & -56.43 & -14.84 \\
\hline Psychodopygus davisi & MT & Santa Carmem & -54.52 & -11.35 \\
\hline Psychodopygus davisi & MT & Santa Rita do Trivelato & -55.26 & -13.81 \\
\hline Psychodopygus davisi & MT & São José do Rio Claro & -56.72 & -13.44 \\
\hline Psychodopygus davisi & MT & São José dos 4 Marcos & -58.17 & -15.17 \\
\hline Psychodopygus davisi & MT & Sinop & -55.49 & -11.83 \\
\hline Psychodopygus davisi & MT & Sorriso & -55.71 & -12.55 \\
\hline Psychodopygus davisi & MT & Taboporã & -56.62 & -10.80 \\
\hline Psychodopygus davisi & MT & Tapurah & -56.50 & -12.53 \\
\hline Psychodopygus davisi & MT & União do Sul & -54.36 & -11.52 \\
\hline Psychodopygus davisi & MT & Vera & -55.19 & -12.18 \\
\hline Psychodopygus davisi & MT & Vila Bela de S. Trindade & -59.95 & -15.00 \\
\hline Psychodopygus davisi & $\mathrm{GO}$ & Bom Jardim & -52.12 & -16.28 \\
\hline Psychodopygus davisi & $\mathrm{GO}$ & Guapó & -49.53 & -16.83 \\
\hline Psychodopygus davisi & $\mathrm{GO}$ & Mambaí & -46.11 & -14.49 \\
\hline Psychodopygus davisi & GO & Maurilândia & -50.31 & -18.00 \\
\hline Psychodopygus davisi & $\mathrm{GO}$ & Posse & -46.37 & -14.09 \\
\hline Psychodopygus davisi & $\mathrm{GO}$ & Itumbiara & -49.21 & -18.42 \\
\hline Psychodopygus davisi & $\mathrm{GO}$ & Mineiros & -50.73 & -16.54 \\
\hline Psychodopygus davisi & $\mathrm{GO}$ & Catalão & -47.95 & -18.17 \\
\hline Psychodopygus davisi & MS & Alcinópolis & -52.87 & -20.44 \\
\hline Psychodopygus davisi & MS & Corguinho & -54.83 & -19.83 \\
\hline Psychodopygus davisi & MS & Rio Verde de Mato Grosso & -54.84 & -18.92 \\
\hline Psychodopygus davisi & MS & Sonora & -54.75 & -17.57 \\
\hline Sciopemyia sordellii & MT & Diamantino & -56.45 & -14.41 \\
\hline
\end{tabular}




\begin{tabular}{|c|c|c|c|c|}
\hline Sandfly species & State code & Municipalities & Longitude & Latitude \\
\hline Sciopemyia sordellii & MT & Varzea Grande & -55.16 & -15.54 \\
\hline Sciopemyia sordellii & MT & Nova Mutum & -56.08 & -13.83 \\
\hline Sciopemyia sordellii & MT & Cuiabá & -56.10 & -15.60 \\
\hline Sciopemyia sordellii & MT & Barra do Garças & -52.26 & -15.89 \\
\hline Sciopemyia sordellii & MT & Acorizal & -56.37 & -15.20 \\
\hline Sciopemyia sordellii & MT & Água Boa & -52.16 & -14.05 \\
\hline Sciopemyia sordellii & MT & Alta Floresta & -56.09 & -9.88 \\
\hline Sciopemyia sordellii & MT & Alto Araguaia & -53.22 & -17.31 \\
\hline Sciopemyia sordellii & MT & Alto da Boa Vista & -51.38 & -11.66 \\
\hline Sciopemyia sordellii & MT & Alto do Paraguai & -56.48 & -14.51 \\
\hline Sciopemyia sordellii & MT & Apiacás & -57.45 & -9.54 \\
\hline Sciopemyia sordellii & MT & Araguaina & -51.83 & -15.73 \\
\hline Sciopemyia sordellii & MT & Arenápolis & -56.82 & -14.43 \\
\hline Sciopemyia sordellii & MT & Aripuanã & -60.63 & -9.17 \\
\hline Sciopemyia sordellii & MT & Barão do Melgaço & -55.97 & -16.19 \\
\hline Sciopemyia sordellii & MT & Barra dos Bugres & -57.18 & -15.08 \\
\hline Sciopemyia sordellii & MT & Brasnorte & -57.98 & -12.15 \\
\hline Sciopemyia sordellii & MT & Campinápolis & -52.88 & -14.50 \\
\hline Sciopemyia sordellii & MT & Campo Novo do Parecis & -57.88 & -13.67 \\
\hline Sciopemyia sordellii & MT & Campo Verde & -55.16 & -15.54 \\
\hline Sciopemyia sordellii & MT & Campos de Julio & -59.08 & -13.53 \\
\hline Sciopemyia sordellii & MT & Canabrava do Norte & -51.83 & -11.03 \\
\hline Sciopemyia sordellii & MT & Canarana & -52.27 & -13.55 \\
\hline Sciopemyia sordellii & MT & Carolina & -55.83 & -9.96 \\
\hline Sciopemyia sordellii & MT & Chapada dos Guimarães & -55.75 & -15.46 \\
\hline Sciopemyia sordellii & MT & Claúdia & -54.88 & -11.49 \\
\hline Sciopemyia sordellii & MT & Colíder & -55.45 & -10.81 \\
\hline Sciopemyia sordellii & MT & Colniza & -59.03 & -9.40 \\
\hline Sciopemyia sordellii & MT & Comodoro & -59.79 & -13.66 \\
\hline Sciopemyia sordellii & MT & Confresa & -51.56 & -10.64 \\
\hline Sciopemyia sordellii & MT & Denise & -57.05 & -14.73 \\
\hline Sciopemyia sordellii & MT & Dom Aquino & -54.55 & -15.48 \\
\hline Sciopemyia sordellii & MT & Gaúcha do Norte & -53.08 & -13.24 \\
\hline Sciopemyia sordellii & MT & Gerneral Carneiro & -52.76 & -15.71 \\
\hline Sciopemyia sordellii & MT & Guarantã do Norte & -54.90 & -9.78 \\
\hline Sciopemyia sordellii & MT & Guiratinga & -53.74 & -16.33 \\
\hline Sciopemyia sordellii & MT & Jaciara & -54.95 & -15.92 \\
\hline Sciopemyia sordellii & MT & Jangada & -56.49 & -15.24 \\
\hline Sciopemyia sordellii & MT & Juína & -58.74 & -11.38 \\
\hline Sciopemyia sordellii & MT & Juscimeira & -54.88 & -16.05 \\
\hline Sciopemyia sordellii & MT & Lucas do Rio Verde & -55.91 & -13.05 \\
\hline Sciopemyia sordellii & MT & Luciara & -50.37 & -10.75 \\
\hline Sciopemyia sordellii & MT & Mercelândia & -54.60 & -11.11 \\
\hline Sciopemyia sordellii & MT & Matupá & -54.90 & -10.05 \\
\hline Sciopemyia sordellii & MT & Mirassol d' Oeste & -58.05 & -15.40 \\
\hline Sciopemyia sordellii & MT & Nobres & -56.33 & -14.73 \\
\hline Sciopemyia sordellii & MT & Nossa S. do Livramento & -56.37 & -15.80 \\
\hline Sciopemyia sordellii & MT & Nova Bandeirantes & -57.86 & -9.95 \\
\hline Sciopemyia sordellii & MT & Nova Brasilândia & -54.57 & -14.57 \\
\hline Sciopemyia sordellii & MT & Nova Canaã do Norte & -55.95 & -10.55 \\
\hline Sciopemyia sordellii & MT & Nova Marilândia & -56.57 & -14.21 \\
\hline Sciopemyia sordellii & MT & Nova Maringá & -57.06 & -13.01 \\
\hline Sciopemyia sordellii & MT & Nova Santana Helena & -53.35 & -10.82 \\
\hline
\end{tabular}




\begin{tabular}{|c|c|c|c|c|}
\hline Sandfly species & State code & Municipalities & Longitude & Latitude \\
\hline Sciopemyia sordellii & MT & Nova Ubiratã & -55.26 & -13.01 \\
\hline Sciopemyia sordellii & MT & Novo Horizonte do Norte & -57.35 & -11.41 \\
\hline Sciopemyia sordellii & MT & Novo São Joaquim & -53.02 & -14.90 \\
\hline Sciopemyia sordellii & MT & Paranaíta & -56.48 & -9.67 \\
\hline Sciopemyia sordellii & MT & Paratinga & -54.05 & -14.43 \\
\hline Sciopemyia sordellii & MT & Pedra Preta & -54.47 & -16.62 \\
\hline Sciopemyia sordellii & MT & Peixoto de Azevedo & -54.98 & -10.22 \\
\hline Sciopemyia sordellii & MT & Planalto da Serra & -55.27 & -10.44 \\
\hline Sciopemyia sordellii & MT & Poconé & -56.62 & -16.26 \\
\hline Sciopemyia sordellii & MT & Pontal do Araguaia & -52.01 & -15.85 \\
\hline Sciopemyia sordellii & MT & Ponte Branca & -56.78 & -16.08 \\
\hline Sciopemyia sordellii & MT & Pontes de Lacerda & -59.37 & -15.20 \\
\hline Sciopemyia sordellii & MT & Porto Alegre do Norte & -51.63 & -10.87 \\
\hline Sciopemyia sordellii & MT & Porto Esperidião & -58.47 & -15.85 \\
\hline Sciopemyia sordellii & MT & Poxoréo & -54.38 & -15.83 \\
\hline Sciopemyia sordellii & MT & Primavera do Leste & -54.30 & -15.56 \\
\hline Sciopemyia sordellii & MT & Querência & -52.11 & -12.35 \\
\hline Sciopemyia sordellii & MT & Ribeirão Cascalheira & -51.82 & -12.92 \\
\hline Sciopemyia sordellii & MT & Ribeirãozinho & -52.67 & -16.45 \\
\hline Sciopemyia sordellii & MT & Rondonópolis & -54.64 & -16.47 \\
\hline Sciopemyia sordellii & MT & Rosário Oeste & -56.43 & -14.84 \\
\hline Sciopemyia sordellii & MT & Santa Carmem & -54.52 & -11.35 \\
\hline Sciopemyia sordellii & MT & Santa Rita do Trivelato & -55.26 & -13.81 \\
\hline Sciopemyia sordellii & MT & Santo Antônio do Leverger & -56.08 & -15.87 \\
\hline Sciopemyia sordellii & MT & São Felix do Araguaia & -50.67 & -11.61 \\
\hline Sciopemyia sordellii & MT & São José do 4 Marcos & -58.17 & -15.17 \\
\hline Sciopemyia sordellii & MT & Serra da Nova Dourado & -51.58 & -11.97 \\
\hline Sciopemyia sordellii & MT & Sinop & -55.49 & -11.83 \\
\hline Sciopemyia sordellii & MT & Sorriso & -55.71 & -12.55 \\
\hline Sciopemyia sordellii & MT & Taboporã & -56.62 & -10.80 \\
\hline Sciopemyia sordellii & MT & Tangará da Serra & -57.50 & -14.62 \\
\hline Sciopemyia sordellii & MT & Terra Nova do Norte & -55.23 & -10.51 \\
\hline Sciopemyia sordellii & MT & Teorixoréu & -52.56 & -16.20 \\
\hline Sciopemyia sordellii & MT & União do Sul & -54.36 & -11.52 \\
\hline Sciopemyia sordellii & MT & Vila Bela de S. Trindade & -59.95 & -15.00 \\
\hline Sciopemyia sordellii & MT & Vila Rica & -51.11 & -10.01 \\
\hline Sciopemyia sordellii & MT & Itanhangá & -56.64 & -12.23 \\
\hline Sciopemyia sordellii & MT & Ipiranga do Norte & -56.05 & -15.35 \\
\hline Sciopemyia sordellii & MT & Vera & -55.19 & -12.18 \\
\hline Sciopemyia sordellii & GO & Itumbiara & -46.25 & -14.80 \\
\hline Sciopemyia sordellii & $\mathrm{GO}$ & Itapaci & -49.57 & -14.95 \\
\hline Sciopemyia sordellii & $\mathrm{DF}$ & Brasília & -47.42 & -15.78 \\
\hline Sciopemyia sordellii & MS & Aparecida do Taboado & -51.09 & -20.09 \\
\hline Sciopemyia sordellii & MS & Aquidauana & -55.79 & -20.47 \\
\hline Sciopemyia sordellii & MS & Bataguassu & -52.42 & -21.71 \\
\hline Sciopemyia sordellii & MS & Bela Vista & -56.52 & -22.11 \\
\hline Sciopemyia sordellii & MS & Bodoquena & -56.72 & -20.54 \\
\hline Sciopemyia sordellii & MS & Campo Grande & -54.65 & -20.44 \\
\hline Sciopemyia sordellii & MS & Corguinho & -54.83 & -19.83 \\
\hline Sciopemyia sordellii & MS & Corumbá & -57.65 & -19.01 \\
\hline Sciopemyia sordellii & MS & Paranaíba & -51.19 & -19.68 \\
\hline Sciopemyia sordellii & MS & Ponta Porã & -55.73 & -22.54 \\
\hline Sciopemyia sordellii & MS & Selvíria & -51.42 & -20.37 \\
\hline
\end{tabular}




\begin{tabular}{|c|c|c|c|c|}
\hline Sandfly species & State code & Municipalities & Longitude & Latitude \\
\hline Sciopemyia sordellii & MS & Terenos & -54.86 & -20.44 \\
\hline Sciopemyia sordellii & MS & Três Lagoas & -51.68 & -20.75 \\
\hline Sciopemyia sordellii & MS & Costa Rica & -53.13 & -18.54 \\
\hline Sciopemyia sordellii & MS & Dourados & -54.81 & -22.22 \\
\hline Sciopemyia sordellii & MS & Guia Lopes da Laguna & -56.11 & -21.46 \\
\hline Sciopemyia sordellii & MS & Itaquiraí & -54.11 & -23.28 \\
\hline Sciopemyia sordellii & MS & Jaraguarí & -54.40 & -20.14 \\
\hline Sciopemyia sordellii & MS & Nova Andradina & -53.34 & -22.23 \\
\hline Sciopemyia sordellii & MS & Novo Horizonte do Sul & -53.80 & -22.61 \\
\hline Sciopemyia sordellii & MS & Nioaque & -55.83 & -21.14 \\
\hline Sciopemyia sordellii & MS & Santa Rita do Pardo & -52.83 & -21.30 \\
\hline Sciopemyia sordellii & MS & Bonito & -41.27 & -11.97 \\
\hline Sciopemyia sordellii & MS & Maracajú & -55.17 & -21.61 \\
\hline Sciopemyia sordellii & MS & Rio Verde de Mato Grosso & -54.84 & -18.92 \\
\hline Sciopemyia sordellii & MS & Aquidauana & -55.79 & -20.47 \\
\hline Sciopemyia sordellii & MS & Nhecolânia & -57.07 & -17.27 \\
\hline Trichopygomyia dasypodogeton & MT & Nova Mutum & -56.08 & -13.83 \\
\hline Trichopygomyia dasypodogeton & MT & Apiacás & -57.45 & -9.54 \\
\hline Trichopygomyia dasypodogeton & MT & Peixoto de Azevedo & -54.98 & -10.22 \\
\hline Trichopygomyia dasypodogeton & MT & Alto Taquarí & -53.16 & -17.49 \\
\hline Trichopygomyia dasypodogeton & MT & Araguaina & -51.83 & -15.73 \\
\hline Trichopygomyia dasypodogeton & MT & Aripuanã & -60.63 & -9.17 \\
\hline Trichopygomyia dasypodogeton & MT & Barra do Garças & -52.26 & -15.89 \\
\hline Trichopygomyia dasypodogeton & MT & Campos de Julio & -59.08 & -13.53 \\
\hline Trichopygomyia dasypodogeton & MT & Claúdia & -54.88 & -11.49 \\
\hline Trichopygomyia dasypodogeton & MT & Colíder & -55.45 & -10.81 \\
\hline Trichopygomyia dasypodogeton & MT & Comodoro & -59.79 & -13.66 \\
\hline Trichopygomyia dasypodogeton & MT & Feliz Natal & -54.92 & -12.39 \\
\hline Trichopygomyia dasypodogeton & MT & Gaúcha do Norte & -53.08 & -13.24 \\
\hline Trichopygomyia dasypodogeton & MT & Lucas do Rio Verde & -55.91 & -13.05 \\
\hline Trichopygomyia dasypodogeton & MT & Mercelândia & -54.60 & -11.11 \\
\hline Trichopygomyia dasypodogeton & MT & Matupá & -54.90 & -10.05 \\
\hline Trichopygomyia dasypodogeton & MT & Nova Marilândia & -56.57 & -14.21 \\
\hline Trichopygomyia dasypodogeton & MT & Nova Maringá & -57.06 & -13.01 \\
\hline Trichopygomyia dasypodogeton & MT & Nova Ubiratã & -55.26 & -13.01 \\
\hline Trichopygomyia dasypodogeton & MT & Paranaíta & -56.48 & -9.67 \\
\hline Trichopygomyia dasypodogeton & MT & Paratinga & -54.05 & -14.43 \\
\hline Trichopygomyia dasypodogeton & MT & Porto Estrela & -57.22 & -15.32 \\
\hline Trichopygomyia dasypodogeton & MT & São Felix do Araguaia & -50.67 & -11.61 \\
\hline Trichopygomyia dasypodogeton & MT & Sinop & -55.49 & -11.83 \\
\hline Trichopygomyia dasypodogeton & MT & Sorriso & -55.71 & -12.55 \\
\hline Trichopygomyia dasypodogeton & MT & Taboporã & -56.62 & -10.80 \\
\hline Trichopygomyia dasypodogeton & MT & União do Sul & -54.36 & -11.52 \\
\hline Trichopygomyia dasypodogeton & MT & Itanhangá & -56.64 & -12.23 \\
\hline
\end{tabular}

DF: Federal District; GO: state of Goiás; MS: state of Mato Grosso do Sul; MT: state of Mato Grosso. 